\title{
2010
}

Oak Ridge National Laboratory Annual Illness and Injury Surveillance Report

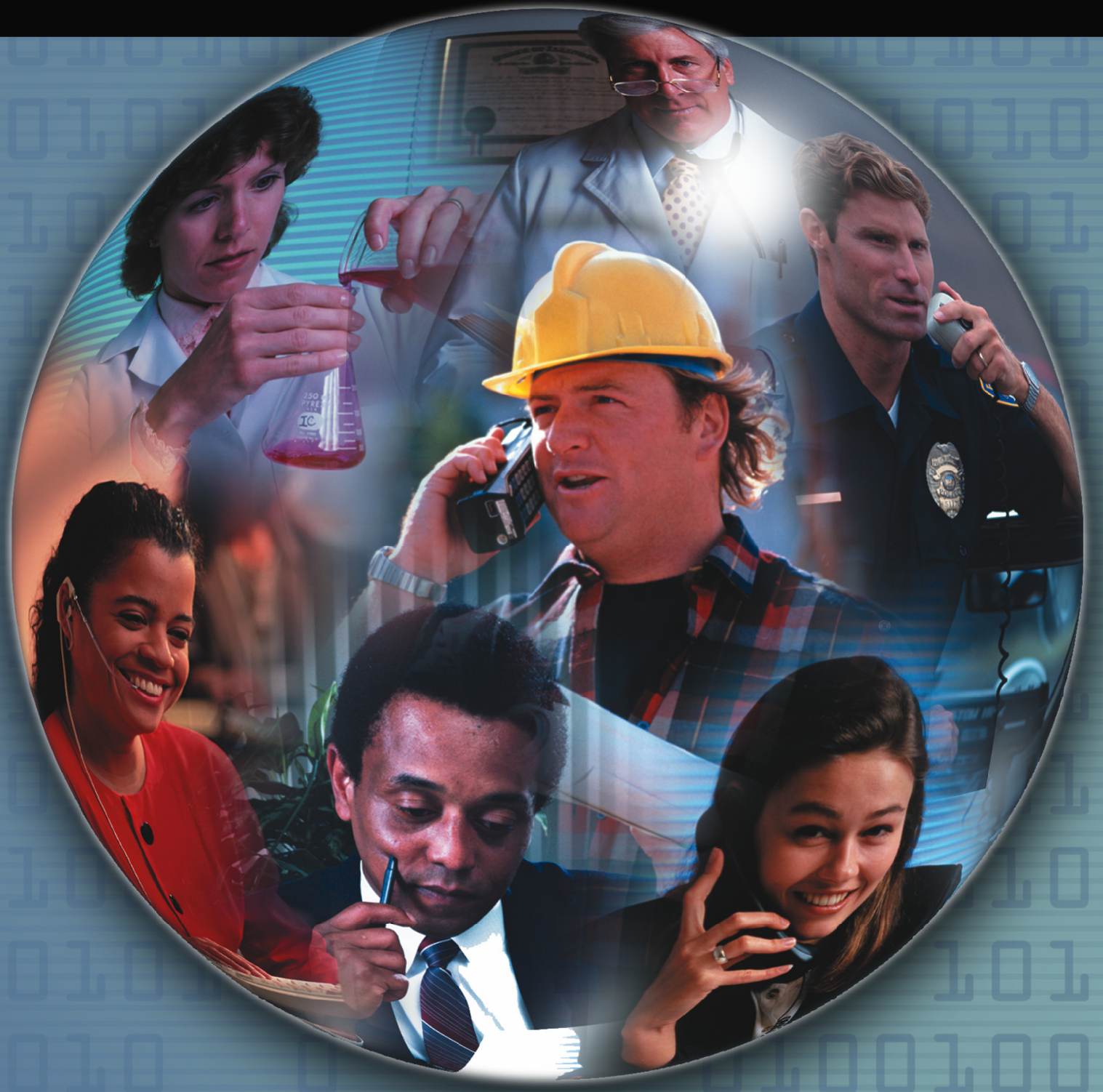




\section{Oak Ridge National Laboratory 2010 Illness and Injury Surveillance Report}

Questions or comments about this report or the Illness and Injury Surveillance Program (IISP) may be directed to:

E-mail:

Dr. Cliff Strader at cliff.strader@hq.doe.gov or Dr. Bonnie Richter at bonnie.richter@hq.doe.gov

or direct letters to:

Mail Stop HS-13 / GTN Building

U.S. Department of Energy

1000 Independence Avenue, S.W.

Washington, DC 20585-0270

Additional information about the Department of Energy's Office of Illness and Injury Prevention Programs, the IISP, and annual reports for DOE sites participating in this program can be found at:

http://www.hss.energy.gov/healthsafety/WSHP/epi/surv/

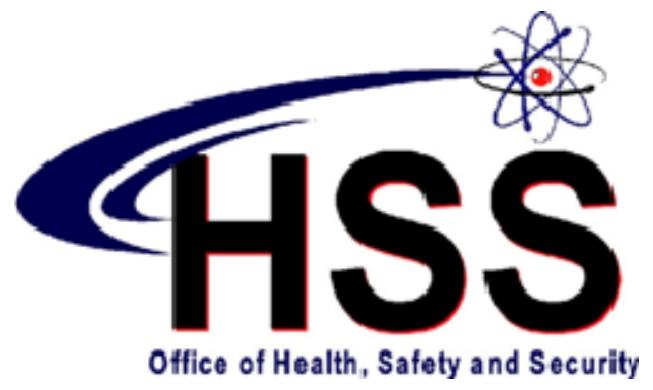

ACKNOWLEDGEMENT

LifeART images copyright 2000 Lippincott Williams \& Wilkins. All rights reserved.

This document was produced under contract number DE-AC05-06OR23100 between the

U.S. Department of Energy and Oak Ridge Associated Universities. 


\title{
Oak Ridge National Laboratory \\ 2010 Illness and Injury Surveillance Report \\ At A Glance
}

\begin{abstract}
NOTE: In previous years, the number of days absent or lost and the age of the work force were reported as averages. Beginning in 2010, they are being reported as medians. A median is the value in a population that half the values exceed and half fall short of it. The median is less affected than the average when the distribution contains a few very large or very small values.
\end{abstract}

Oak Ridge National Laboratory (ORNL) reported 403 absences among 1,505 female workers in 2010; an absence rate of 26.8 per 100 workers $(403 / 1,505)$. There were 572 absences among 3,552 male workers; an absence rate of 16.1 per 100 workers $(572 / 3,552)$. Absence rates in 2010 decreased more than 6 percent for both men and women compared with 2009. Absence rates among women have remained higher than those of men since 2006, consistent with the overall trend for all IISP sites combined from 1999 to 2008.

Respiratory diseases, musculoskeletal conditions, and digestive conditions accounted for 51 percent of all diagnoses for women. Injuries and musculoskeletal and digestive conditions accounted for 52 percent of all diagnoses for men. Injuries and disorders of the musculoskeletal and respiratory systems have been among the most frequently reported diagnoses since 2006 for both women and men at ORNL. The most commonly reported diagnoses in each of these categories were upper respiratory conditions (respiratory diseases), joint disorders (musculoskeletal conditions), and inflammation of the intestines (digestive conditions) among women and sprains and strains (injuries), joint disorders (musculoskeletal conditions), and inflammation of the intestines (digestive conditions) among men.

Women lost 8,402 calendar days and men lost 13,999 calendar days due to illness and injury in 2010. The median length of absence was 11 days for women and 12 days for men. 
Security and Fire workers had the highest absence rates in 2010 for men and Crafts workers had the highest absence rate for women. The median length of absences among these workers was 19 days for male Security and Fire workers, 7 more days than the 12-day median absence for all male workers, and 32 days for female Crafts workers, 21 days longer than the 11-day median absence among all female workers in 2009.

Fourteen possible and 1 definite sentinel health events for occupations (diseases likely to be occupationally related) were reported by 7 men and 7 women in 2010. Nine diagnoses were for carpal tunnel syndrome, reported by 5 men and 3 women, with all but 2 aged 50 or older. Of the 8 workers reporting carpal tunnel syndrome, 4 were in the Professional job category. The remaining 4 workers were in 3 job categories.

Forty-four OSHA-recordable events were reported in 2010 (15 reported by women and 29 reported by men), a 29 percent decrease in the number of events reported in 2009, producing a rate of less than 1 event per 100 workers among men and women.

Technical Support workers had the highest rate of OSHA diagnoses among women (54 per 1,000 workers). Administrative Support workers had the highest rate among men (394 per 1,000 workers). Female Technical Support workers reported a total of 10 OSHA diagnoses, 7 due to injuries (mainly unspecified injures). Male Administrative Support workers reported 5 diagnoses distributed among several diagnostic categories.

The most common types of accident that resulted in an OSHA-recordable event involved falls among women and overexertion/strenuous movements or being struck by an object among men. 


\section{The Oak Ridge National Laboratory Work Force - 2010}

The Work Force by Gender and Age 1

The Work Force by Gender and Job

Category 1

Number and Length of Absences

Absence Rate by Gender and Age 2

Number of Days Absent by

Gender and Age

Absence Rate by Job Category

and Gender 3

Median Duration of Absence by

Job Category and Gender. ... 3

\section{Diagnostic Categories}

Number of Diagnoses and Lost Calendar Days by Diagnostic Category

(Categorized by ICD-9-CM) and Gender

Common Diagnoses Among Female

Workers in 2010

Common Diagnoses Among Male

Workers in 2010 .. 6

Number of Most Frequently Reported

Diagnoses by Job Category and Gender 7

\section{Rates of Disease Occurrence}

Rates for All Illnesses and Injuries Combined by Job Category, Gender, and Age

Rates for Selected Diagnostic Categories by Job Category, Gender, and Age .. 8

\section{Time Trends}

Age-Adjusted Rates for All Diagnoses Combined Among Women and Men from 2006 to 2010
Age-Adjusted Rates for Selected Diagnostic Categories Among Women and Men from 2006 to 2010

Age-Adjusted Rates for All Diagnoses

Combined Among Women and Men by Job

Category from 2006 to $2010 \ldots \ldots \ldots \ldots \ldots \ldots \ldots \ldots . \ldots 12$

\section{Sentinel Health Events for Occupations (SHEOs)}

Characteristics of SHEOs by Gender 13

SHEO Diagnoses by Gender 13

Occupational Safety and Health Administration (OSHA)-Recordable Events

OSHA-Recordable Events by Gender and Age. 14

OSHA-Recordable Events by Job

Category and Gender 14

\section{Diagnostic and Accident Categories for OSHA-Recordable Events}

OSHA-Recordable Diagnoses by

Diagnostic Category and Gender

OSHA-Recordable Accidents by Type

and Gender

Rates of OSHA-Recordable Events

OSHA-Recordable Rates by Age and Job

Categories Among Women, All Diagnoses

Combined

OSHA-Recordable Rates by Age and Job

Categories Among Men, All Diagnoses

Combined

Time Trends for OSHA-Recordable Events

Age-Adjusted Rates for All OSHA-Recordable Diagnoses Combined Among Women and Men by Job Category from 2006 to 2010 .

\section{Appendices}


The Oak Ridge National Laboratory Work Force - 2010

Figure 1. The Work Force by Gender and Age

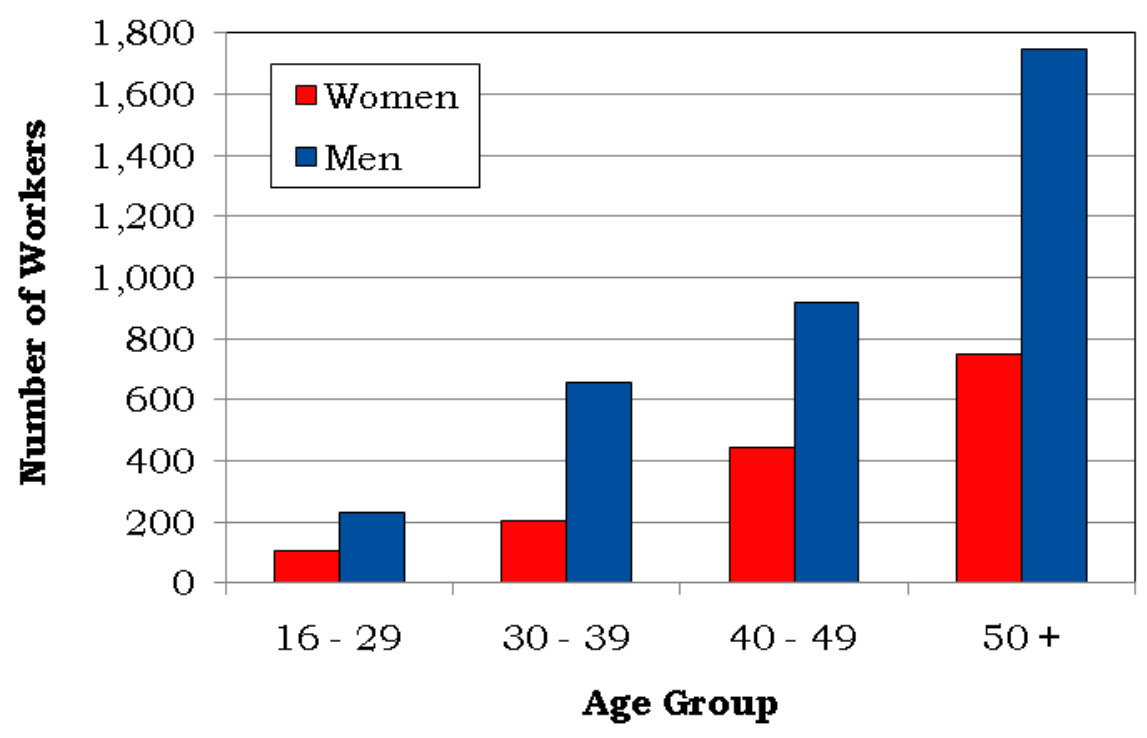

Figure 2. The Work Force by Gender and Job Category

\begin{tabular}{|l|c|c|}
\hline \multicolumn{1}{|c|}{ Job Category } & W omen & M en \\
\hline Professional & $\mathbf{7 6 0}$ & $\mathbf{2 , 5 6 3}$ \\
& $\mathbf{5 0 \%}$ & $\mathbf{7 2 \%}$ \\
\hline \multirow{2}{*}{ Administrative Support } & $\mathbf{5 2 9}$ & $\mathbf{2 0}$ \\
& $\mathbf{3 5 \%}$ & $\mathbf{1} \%$ \\
\hline \multirow{2}{*}{ Technical Support } & $\mathbf{1 2 6}$ & $\mathbf{3 1 1}$ \\
& $\mathbf{8} \%$ & $\mathbf{9 \%}$ \\
\hline \multirow{2}{*}{ Service } & $\mathbf{4 7}$ & $\mathbf{1 0 9}$ \\
& $\mathbf{3} \%$ & $\mathbf{3} \%$ \\
\hline \multirow{2}{*}{ Security and Fire } & $\mathbf{9}$ & $\mathbf{3 4}$ \\
& $\mathbf{1} \%$ & $\mathbf{1} \%$ \\
\hline \multirow{2}{*}{ Crafts } & $\mathbf{3 2}$ & $\mathbf{4 6 0}$ \\
& $\mathbf{2 \%}$ & $\mathbf{1 3} \%$ \\
\hline \multirow{2}{*}{ Line Operators } & $\mathbf{2}$ & $\mathbf{5 5}$ \\
& $<\mathbf{1 \%}$ & $\mathbf{1} \%$ \\
\hline \multirow{2}{*}{ Total } & $\mathbf{1 , 5 0 5}$ & $\mathbf{3 , 5 5 2}$ \\
& $100 \%$ & $\mathbf{1 0 0} \%$ \\
\hline
\end{tabular}




\section{Number and Length of Absences}

Figure 3. Absence Rate by Gender and Age

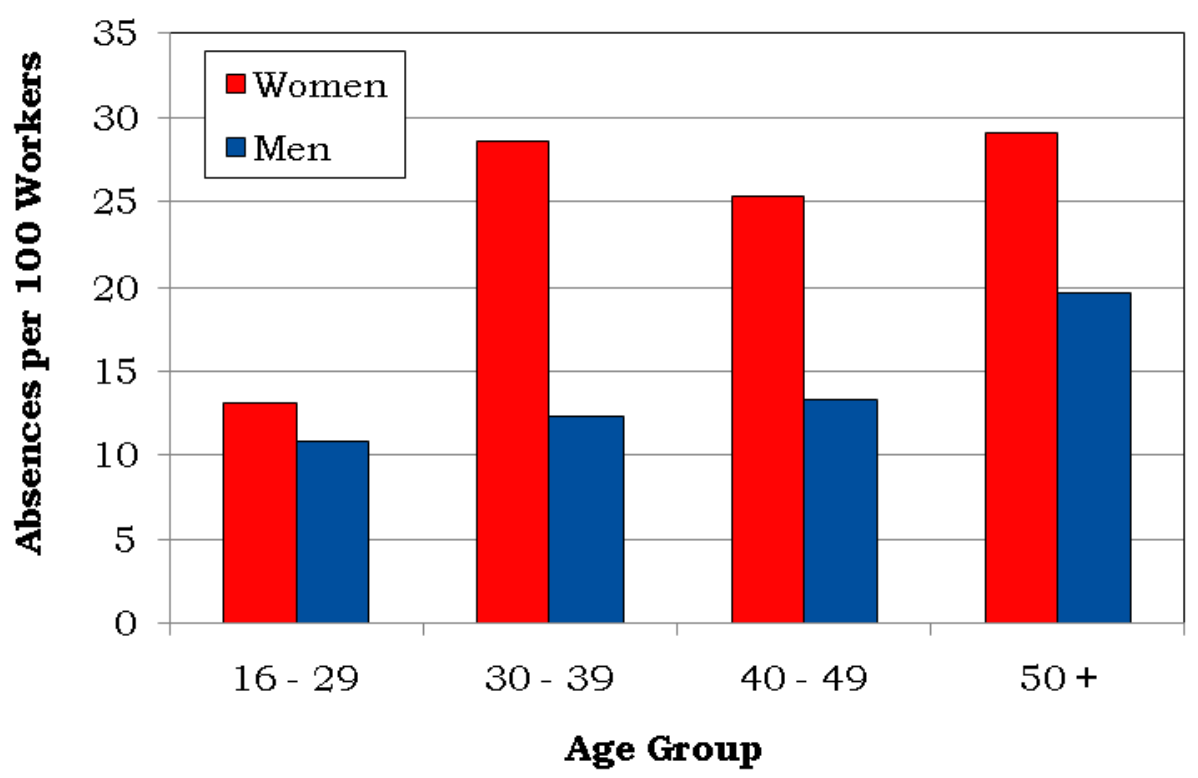

Figure 4. Number of Days Absent by Gender and Age

\begin{tabular}{|c|c|c|c|c|}
\hline \multirow{3}{*}{ Gender } & \multirow{2}{*}{ Age } & \multirow{2}{*}{$\begin{array}{c}\text { Number of } \\
\text { Absences }\end{array}$} & \multicolumn{2}{|c|}{ Number of Days Absent } \\
\cline { 3 - 5 } & & 14 & Total & Median \\
\hline \multirow{4}{*}{ Women } & $16-29$ & 59 & 139 & 8 \\
\cline { 2 - 5 } & $30-39$ & 112 & 2,132 & 10 \\
\cline { 2 - 5 } & $40-49$ & 218 & 4,783 & 8 \\
\cline { 2 - 5 } & $50+$ & 403 & 8,402 & 14 \\
\cline { 2 - 5 } & Total & 25 & 299 & 11 \\
\hline \multirow{4}{*}{ Men } & $16-29$ & 81 & 2,022 & 12 \\
\cline { 2 - 5 } & $30-39$ & 122 & 2,678 & 10 \\
\cline { 2 - 5 } & $40-49$ & 344 & 9,000 & 14 \\
\cline { 2 - 5 } & $50+$ & 572 & 13,999 & 12 \\
\cline { 2 - 5 } & Total & & & \\
\cline { 2 - 5 }
\end{tabular}

*Normal pregnancies were excluded from absences for women. 
Figure 5. Absence Rate by Job Category and Gender

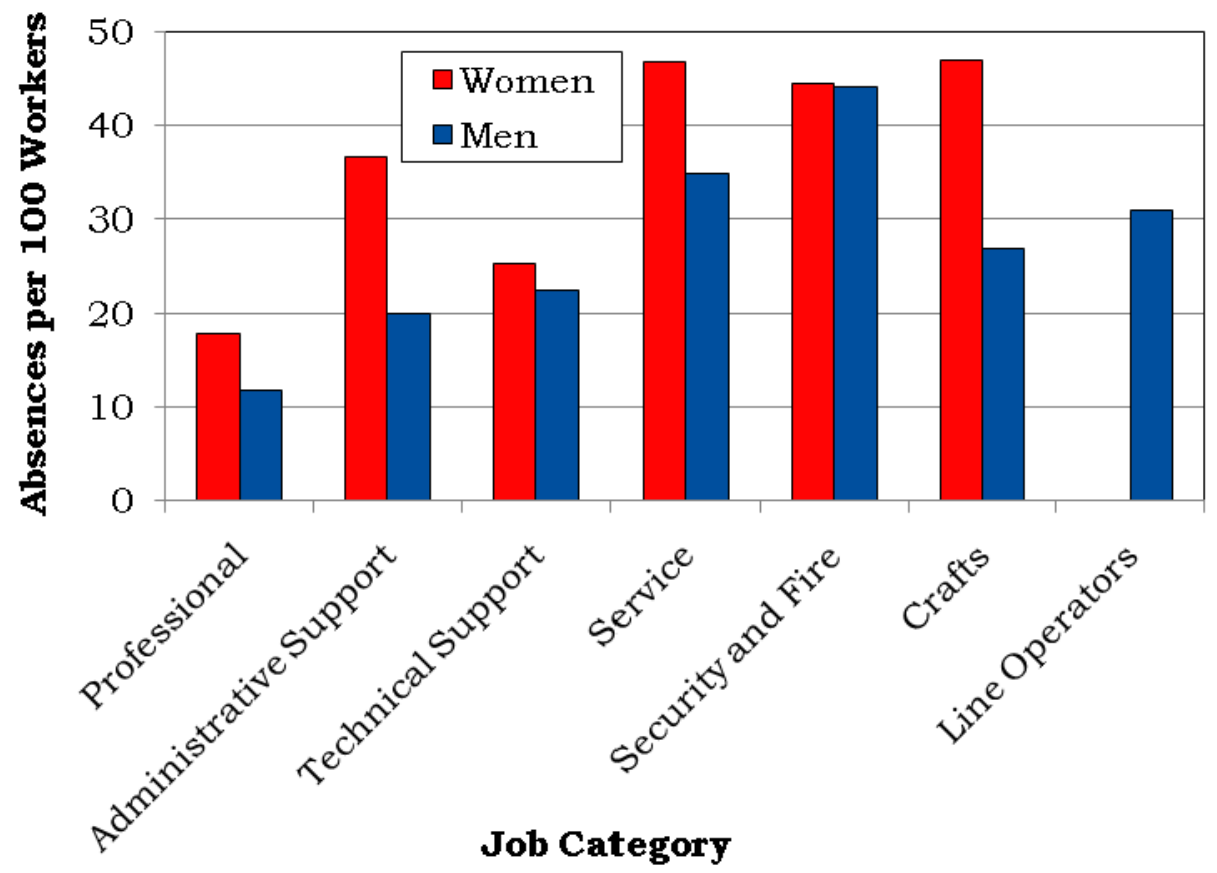

Figure 6. Median Duration of Absence by Job Category and Gender

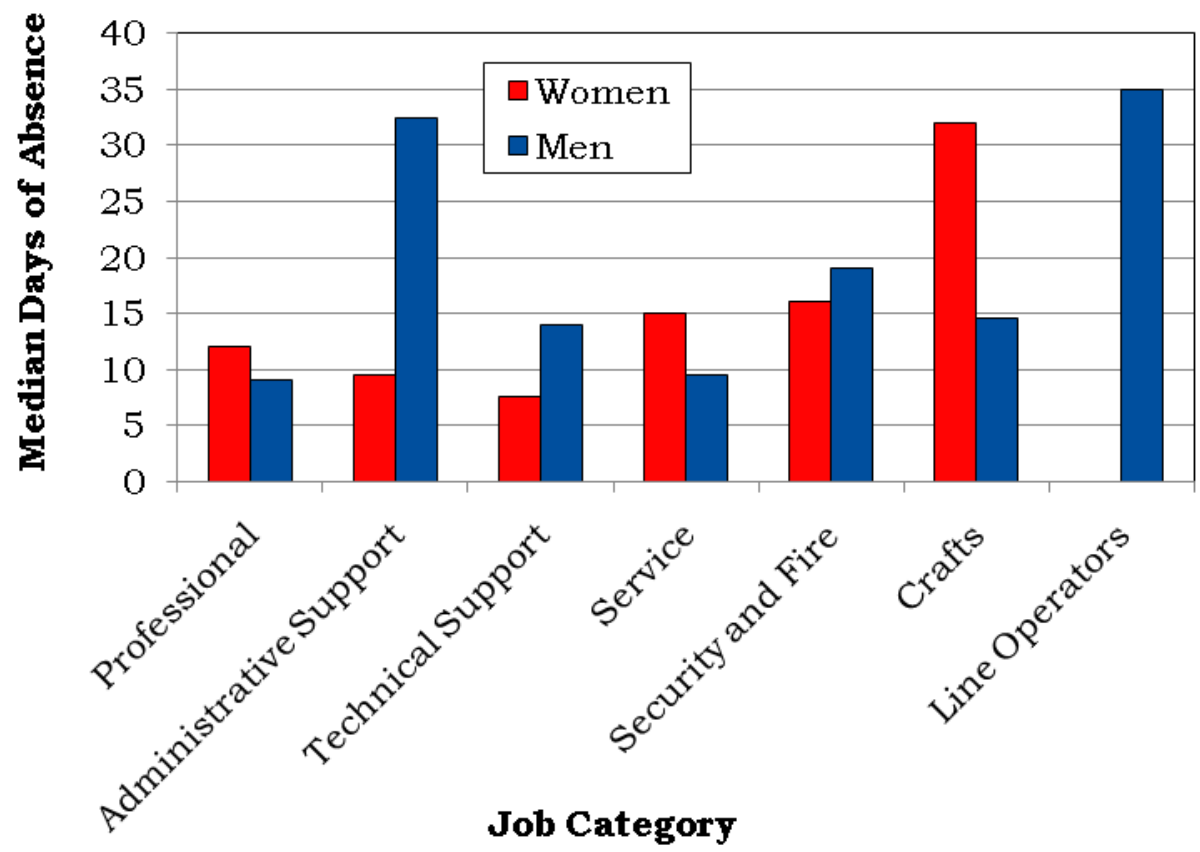




\section{Diagnostic Categories}

Figure 7. Number of Diagnoses and Lost Calendar Days by Diagnostic Category (Categorized by ICD-9-CM) and Gender

\begin{tabular}{|c|c|c|c|c|}
\hline \multirow[b]{2}{*}{ Diagnostic Category } & \multicolumn{2}{|c|}{ Women } & \multicolumn{2}{|c|}{ Men } \\
\hline & $\begin{array}{l}\text { Number of } \\
\text { Diagnoses }\end{array}$ & $\begin{array}{c}\text { Number } \\
\text { of Lost } \\
\text { Calendar } \\
\text { Days }\end{array}$ & $\begin{array}{l}\text { Number of } \\
\text { Diagnoses }\end{array}$ & $\begin{array}{c}\text { Number } \\
\text { of Lost } \\
\text { Calendar } \\
\text { Days }\end{array}$ \\
\hline Benign Growths & 11 & 243 & 16 & 255 \\
\hline Blood & 2 & 19 & 0 & 0 \\
\hline Cancer & 13 & 245 & 7 & 396 \\
\hline Digestive & 73 & 945 & 114 & 1,884 \\
\hline Endocrine / Me tabolic & 7 & 101 & 10 & 164 \\
\hline Existing Birth Condition & 1 & 22 & 1 & 3 \\
\hline Genitourinary & 40 & 894 & 32 & 455 \\
\hline Heart/Circulatory & 13 & 324 & 62 & 1,211 \\
\hline Infections/Parasites & 19 & 236 & 26 & 248 \\
\hline Injury & 67 & 1,624 & 164 & 4,291 \\
\hline Miscarriage & 0 & 0 & NA & NA \\
\hline Musculoskeletal & 86 & 3,031 & 130 & 5,193 \\
\hline Nervous System & 41 & 758 & 49 & 957 \\
\hline Psychological & 9 & 529 & 14 & 369 \\
\hline Respiratory & 121 & 939 & 92 & 871 \\
\hline Skin & 8 & 215 & 14 & 693 \\
\hline Unspecified Symptoms & 33 & 345 & 51 & 547 \\
\hline
\end{tabular}

Note: Lost calendar days for each absence are counted more than once when multiple diagnoses occur in different diagnostic categories for the same absence. 


\section{Figure 8. Common Diagnoses Among Female Workers in 2010}

Intestinal Disorders

Hernias

Gallbladder

Disorders Esophagus/Stomach Appendicitis

All Other

Kidney Disorders

Disorders of the Reproductive Organs

Bladder Disorders

All Other

Sprains and Strains

Open Wounds

Fractures

Dislocations

Bruises

All Other

Rheumatism

Joints

Back

All Other

Upper Respiratory

Pneumonia/Flu

Bronchitis/Asthma

All Other

Headache

Fatigue

Dizziness

Digestive Symptoms

Chest Pain

All Other
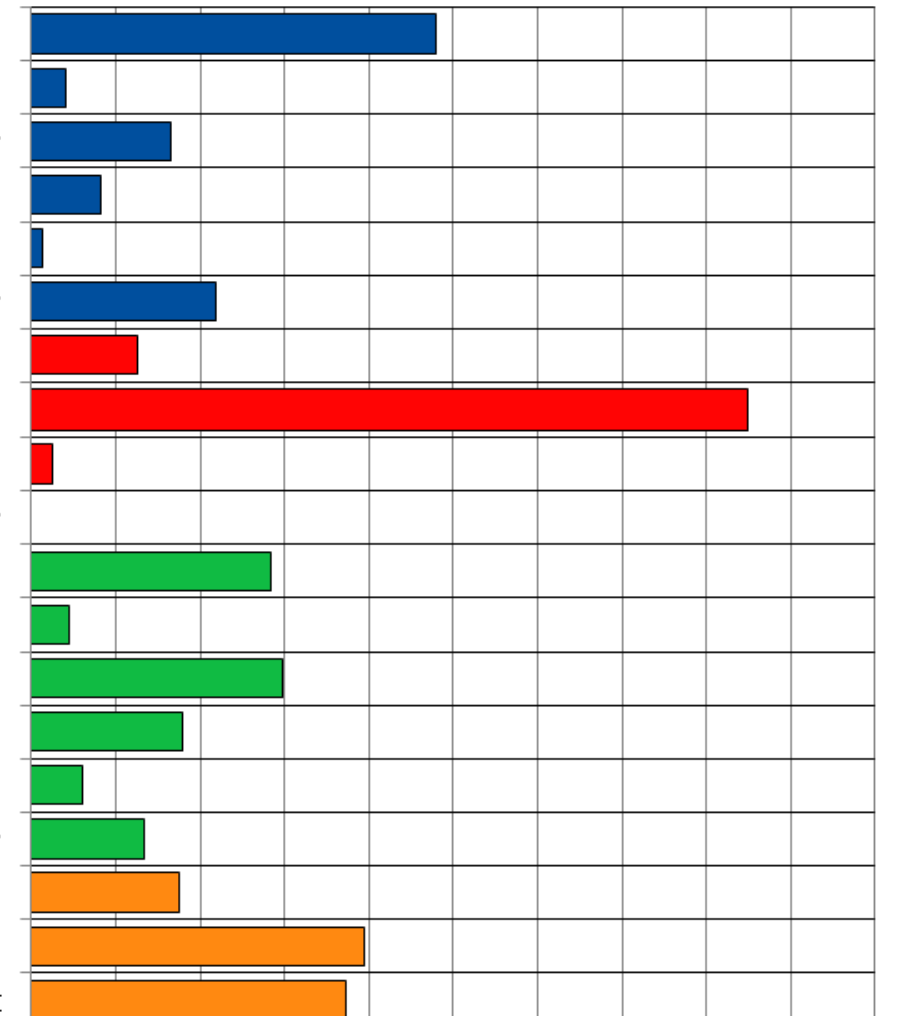

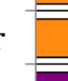

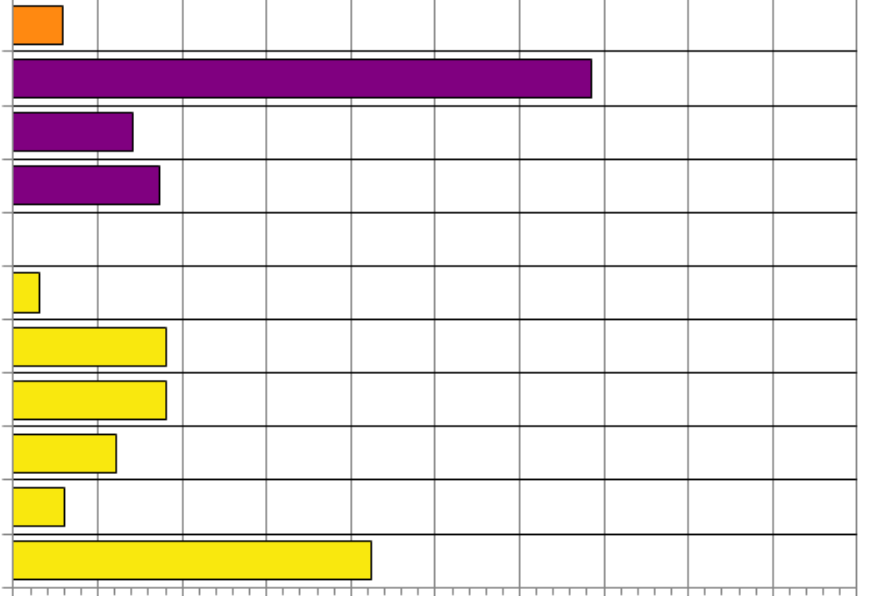

$\begin{array}{lllllllllll}0 & 10 & 20 & 30 & 40 & 50 & 60 & 70 & 80 & 90 & 100\end{array}$

Percent Distribution of Diagnoses Within Diagnostic Category

Dige stive, 73 Diagnoses

Genitourinary, 40 Diagnoses

Injury, 67 Diagnoses
Musculoskeletal, 86 Diagnoses

Respiratory, 121 Diagnoses

Unspecified Symptoms, 33 Diagnoses 


\section{Figure 9. Common Diagnoses Among Male Workers in 2010}

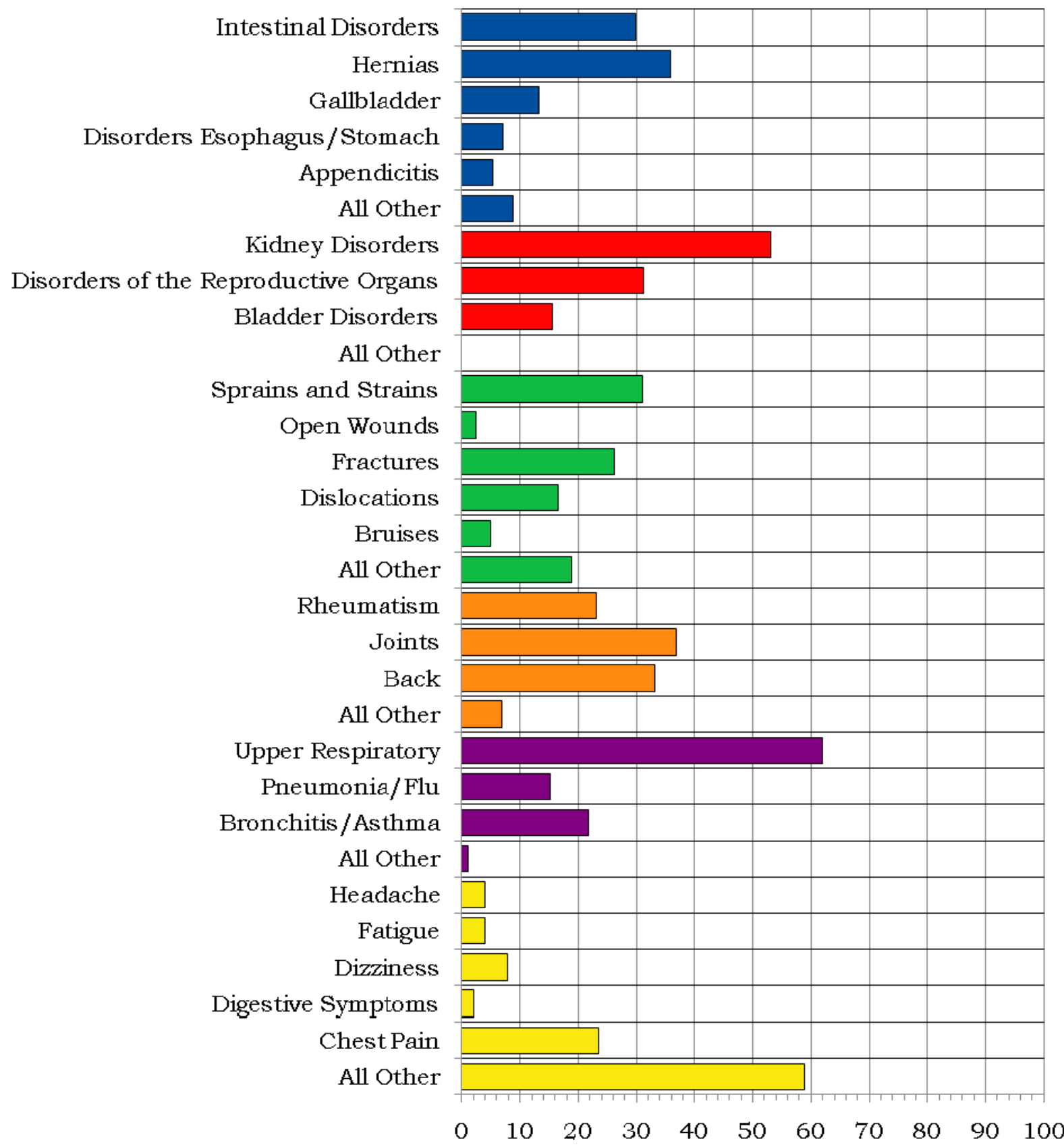

Percent distribution of Diagnoses Within Diagnostic Category

Digestive, 114 Diagnoses

Genitourinary, 32 Diagnoses

Injury, 164 Diagnoses
Musculoskeletal, 130 Diagnoses

Respiratory, 92 Diagnoses

Unspecified Symptoms, 51 Diagnoses 


\section{Figure 10. Number of Most Frequently Reported Diagnoses by Job Category and Gender}

\begin{tabular}{|c|c|c|c|c|}
\hline Job Category & \multicolumn{2}{|l|}{ Men } & \multicolumn{2}{|l|}{ Women } \\
\hline \multirow{3}{*}{ Professional } & Injury & 96 & Respiratory & 39 \\
\hline & Musculoskeletal & 70 & Injury & 32 \\
\hline & Digestive & 53 & M usculoskeletal & 21 \\
\hline \multirow{3}{*}{ Administrative Support } & Psychological & 2 & Respiratory & 67 \\
\hline & Injury & 1 & Digestive & 46 \\
\hline & Digestive & 1 & M usculoskeletal & 40 \\
\hline \multirow{4}{*}{ Technical Support } & Digestive & 21 & M usculoskeletal & 8 \\
\hline & Injury & 15 & Respiratory & 7 \\
\hline & Musculoskeletal & 13 & Injury & 6 \\
\hline & Unspecified Symptoms & 13 & & \\
\hline \multirow{4}{*}{ Service } & Digestive & 9 & M usculoskeletal & 9 \\
\hline & Respiratory & 9 & Respiratory & 5 \\
\hline & Musculoskeletal & 6 & Digestive & 3 \\
\hline & & & Genitourinary & 3 \\
\hline \multirow{4}{*}{ Security and Fire } & Digestive & 5 & Respiratory & 2 \\
\hline & Injury & 4 & Nervous System & 2 \\
\hline & M usculoskeletal & 4 & Endocrine/M etabolic & 1 \\
\hline & Respiratory & 4 & & \\
\hline \multirow{4}{*}{ Crafts } & Injury & 37 & M usculoskeletal & 8 \\
\hline & M usculoskeletal & 31 & Injury & 3 \\
\hline & Respiratory & 24 & Digestive & 2 \\
\hline & & & Genitourinary & 2 \\
\hline \multirow{3}{*}{ Line Operators } & Injury & 6 & & 0 \\
\hline & M usculoskeletal & 6 & & \\
\hline & Nervous System & 5 & & \\
\hline
\end{tabular}




\section{Rates of Disease Occurrence}

Figure 11. Rates for All Illnesses and Injuries Combined by Job Category, Gender, and Age

\begin{tabular}{|c|c|c|c|c|}
\hline \multirow{2}{*}{$\begin{array}{c}\text { All Illnesses \& } \\
\text { Injuries Combined }\end{array}$} & \multicolumn{4}{|c|}{ Rate per 1,000} \\
\hline & Job Category & Age & Men & Women \\
\hline & \multirow{2}{*}{ Professional } & $<50$ & 110 & 212 \\
\hline & & $50+$ & 212 & 285 \\
\hline & \multirow{2}{*}{ Administrative Support } & $<50$ & 91 & 514 \\
\hline & & $50+$ & 333 & 510 \\
\hline & \multirow{2}{*}{ Technical Support } & $<50$ & 247 & 261 \\
\hline & & $50+$ & 336 & 298 \\
\hline & \multirow{2}{*}{ Service } & $<50$ & 365 & 765 \\
\hline & & $50+$ & 543 & 567 \\
\hline & \multirow{2}{*}{ Security and Fire } & $<50$ & 682 & 0 \\
\hline & & $50+$ & 667 & 1,250 \\
\hline & \multirow{2}{*}{ Crafts } & $<50$ & 280 & 667 \\
\hline & & $50+$ & 482 & 353 \\
\hline & \multirow{2}{*}{ Line Operators } & $<50$ & 486 & 0 \\
\hline & & $50+$ & 550 & 0 \\
\hline
\end{tabular}

Figure 12. Rates for Selected Diagnostic Categories by Job Category, Gender, and Age

\begin{tabular}{|c|c|c|c|c|}
\hline \multirow{2}{*}{ Cancer } & \multicolumn{4}{|c|}{ Rate per 1,000} \\
\hline & Job Category & Age & Men & Women \\
\hline & Professional & $<50$ & 0 & 7 \\
\hline & 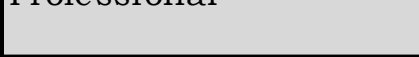 & $50+$ & 5 & 20 \\
\hline & Administrative Support & $<50$ & 0 & 8 \\
\hline & & $50+$ & 0 & 0 \\
\hline & Technical Support & $<50$ & 0 & 0 \\
\hline & & $50+$ & 8 & 18 \\
\hline & Service & $<50$ & 0 & 0 \\
\hline & & $50+$ & 0 & 0 \\
\hline & Security and Fire & $<50$ & 0 & 0 \\
\hline & & $50+$ & 0 & 0 \\
\hline & Crafts & $<50$ & 0 & 0 \\
\hline & Craits & $50+$ & 0 & 0 \\
\hline & Line $\mathrm{Op}$ & $<50$ & 0 & 0 \\
\hline & & $50+$ & 0 & 0 \\
\hline
\end{tabular}


Figure 12. Rates for Selected Diagnostic Categories by Job Category, Gender, and Age (Continued)

\begin{tabular}{|l|l|c|c|c|}
\hline \multirow{2}{*}{ Heart/Circulatory } & \multicolumn{4}{|c|}{ Rate per 1,000 } \\
\cline { 2 - 5 } & \multicolumn{1}{|c|}{ Job Category } & Age & M en & W omen \\
\cline { 2 - 5 } & Professional & $<50$ & $\mathbf{9}$ & $\mathbf{0}$ \\
\cline { 2 - 5 } & $50+$ & $\mathbf{2 7}$ & $\mathbf{1 4}$ \\
\hline \multirow{2}{*}{ Administrative Support } & $<50$ & $\mathbf{0}$ & $\mathbf{4}$ \\
\cline { 2 - 5 } & $50+$ & $\mathbf{0}$ & $\mathbf{2 1}$ \\
\cline { 2 - 5 } & \multirow{2}{*}{ Technical Support } & $<50$ & $\mathbf{0}$ & $\mathbf{0}$ \\
\cline { 2 - 5 } & & $50+$ & $\mathbf{2 4}$ & $\mathbf{0}$ \\
\cline { 2 - 5 } & \multirow{2}{*}{ Service } & $<50$ & $\mathbf{2 7}$ & $\mathbf{5 9}$ \\
\cline { 2 - 5 } & $50+$ & $\mathbf{0}$ & $\mathbf{0}$ \\
\cline { 2 - 5 } & \multirow{2}{*}{ Security and Fire } & $<50$ & $\mathbf{0}$ & $\mathbf{0}$ \\
\cline { 2 - 5 } & $50+$ & $\mathbf{8 3}$ & $\mathbf{0}$ \\
\cline { 2 - 5 } & \multirow{2}{*}{ Crafts } & $<50$ & $\mathbf{0}$ & $\mathbf{0}$ \\
\cline { 2 - 5 } & $50+$ & $\mathbf{3 2}$ & $\mathbf{0}$ \\
\cline { 2 - 5 } & \multirow{2}{*}{ Line Operators } & $<50$ & $\mathbf{0}$ & $\mathbf{0}$ \\
\cline { 2 - 5 } & $50+$ & $\mathbf{5 0}$ & $\mathbf{0}$ \\
\hline
\end{tabular}

\begin{tabular}{|c|c|c|c|c|}
\hline \multirow{2}{*}{ Respiratory } & \multicolumn{4}{|c|}{ Rate per 1,000} \\
\hline & Job Category & Age & Men & Women \\
\hline & \multirow{2}{*}{ Professional } & $<50$ & 14 & 59 \\
\hline & & $50+$ & 16 & 42 \\
\hline & \multirow{2}{*}{ Administrative Support } & $<50$ & 0 & 128 \\
\hline & & $50+$ & 0 & 126 \\
\hline & \multirow{2}{*}{ Technical Support } & $<50$ & 38 & 72 \\
\hline & & $50+$ & 40 & 35 \\
\hline & \multirow{2}{*}{ Service } & $<50$ & 41 & 176 \\
\hline & & $50+$ & 171 & 67 \\
\hline & \multirow{2}{*}{ Security and Fire } & $<50$ & 0 & 0 \\
\hline & & $50+$ & 333 & 500 \\
\hline & \multirow{2}{*}{ Crafts } & $<50$ & 52 & 0 \\
\hline & & $50+$ & 52 & 59 \\
\hline & \multirow{2}{*}{ Line Operators } & $<50$ & 57 & 0 \\
\hline & & $50+$ & 100 & 0 \\
\hline
\end{tabular}


Figure 12. Rates for Selected Diagnostic Categories by Job Category, Gender, and Age (Continued)

\begin{tabular}{|c|c|c|c|c|}
\hline \multirow{2}{*}{ Injury } & \multicolumn{4}{|c|}{ Rate per 1,000 } \\
\hline & Job Category & Age & Men & Women \\
\hline & \multirow{2}{*}{ Professional } & $<50$ & 25 & 30 \\
\hline & & $50+$ & 49 & 56 \\
\hline & \multirow{2}{*}{ Administrative Support } & $<50$ & 91 & 37 \\
\hline & & $50+$ & 0 & 52 \\
\hline & \multirow{2}{*}{ Technical Support } & $<50$ & 54 & 43 \\
\hline & & $50+$ & 40 & 53 \\
\hline & \multirow{2}{*}{ Service } & $<50$ & 41 & 59 \\
\hline & & $50+$ & 57 & 33 \\
\hline & \multirow{2}{*}{ Security and Fire } & $<50$ & 182 & 0 \\
\hline & & $50+$ & 0 & 0 \\
\hline & \multirow{2}{*}{ Crafts } & $<50$ & 71 & 133 \\
\hline & & $50+$ & 88 & 59 \\
\hline & \multirow{2}{*}{ Line Operators } & $<50$ & 114 & 0 \\
\hline & & $50+$ & 100 & 0 \\
\hline
\end{tabular}

Time Trends

Figure 13. Age-Adjusted Rates for All Diagnoses Combined Among Women and Men from 2006 to 2010*

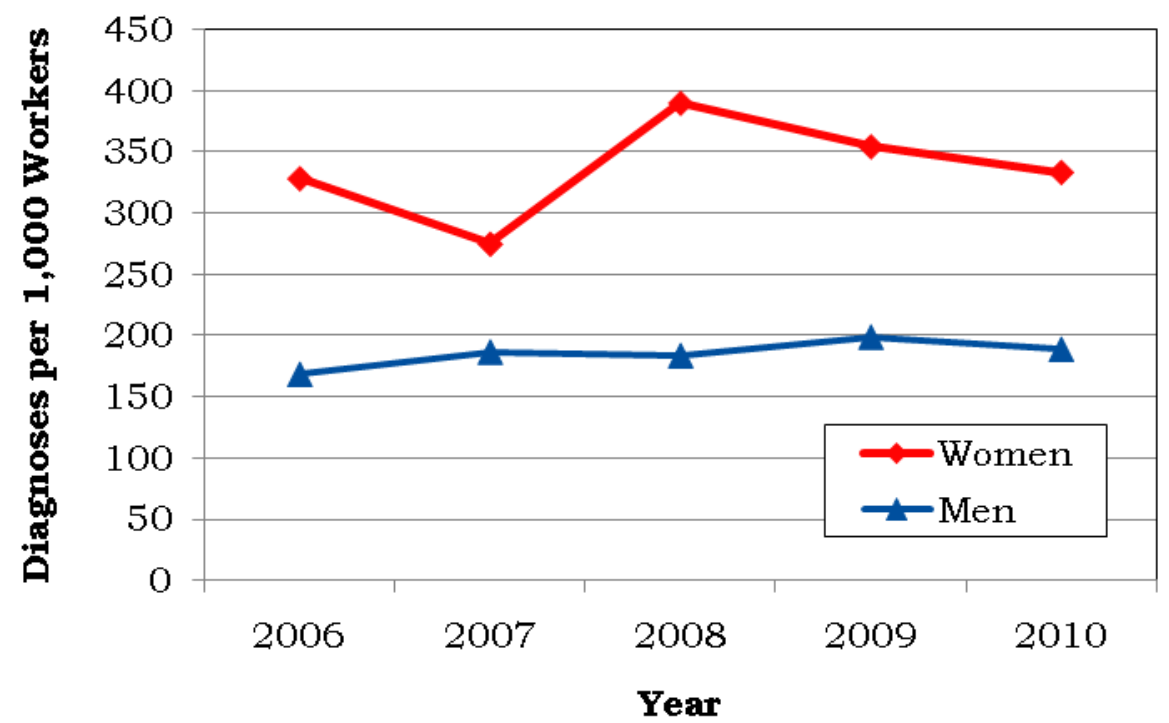

*Standardized to age distribution of 2000 U.S. population. 
Figure 14. Age-Adjusted Rates for Selected Diagnostic Categories Among Women and Men from 2006 to 2010*

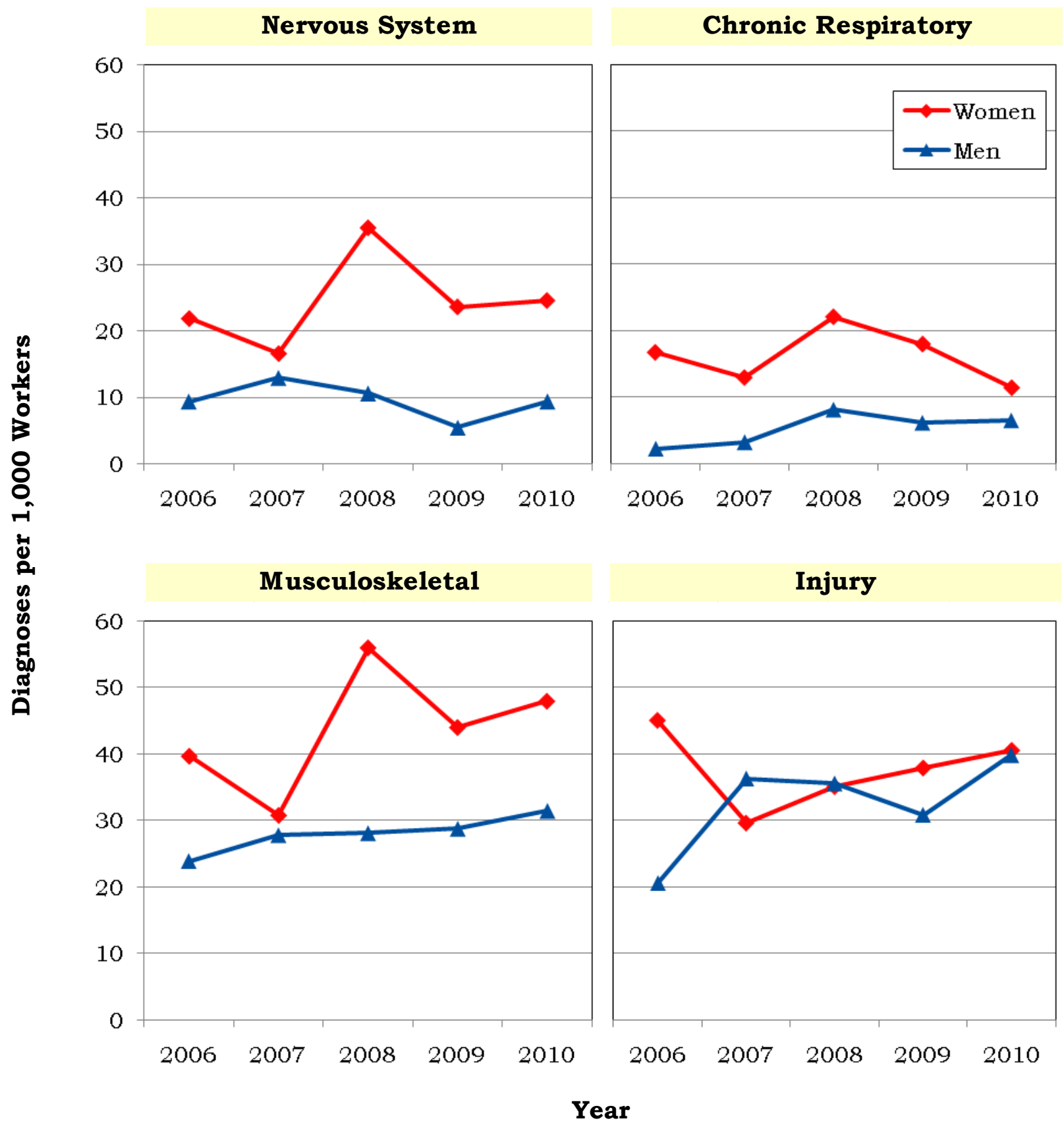

*Standardized to age distribution of 2000 U.S. population. 
Figure 15. Age-Adjusted Rates for All Diagnoses Combined Among Women and Men by Job Category from 2006 to 2010*

Professional

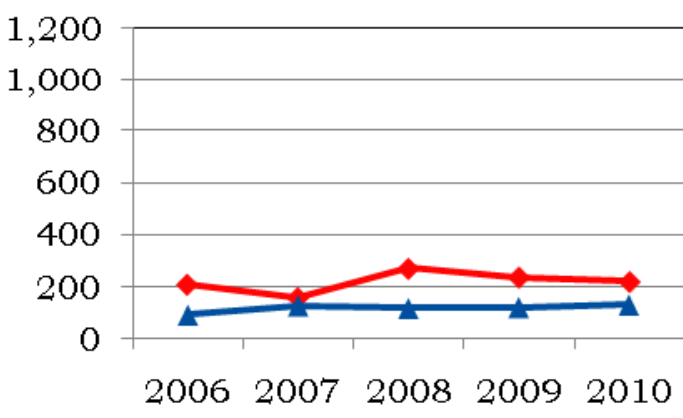

Administrative Support

वे

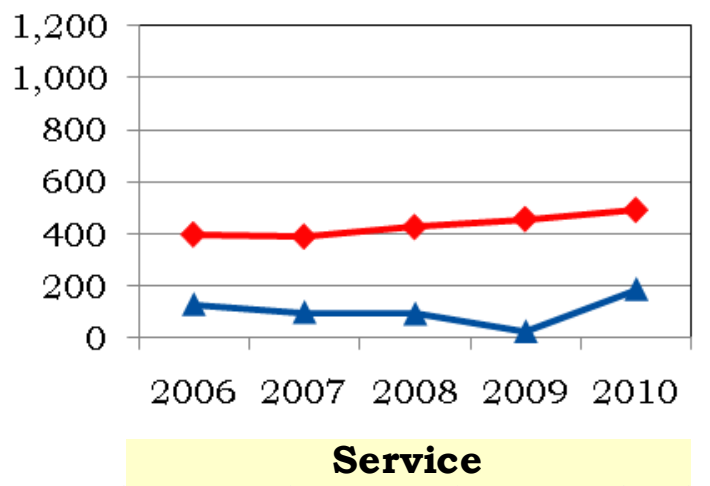

Technical Support

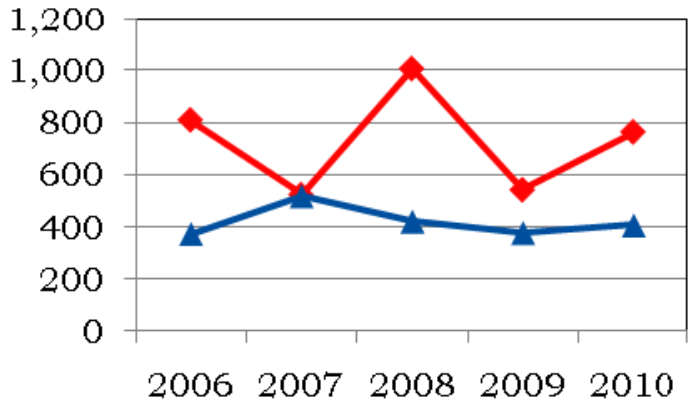

Crafts

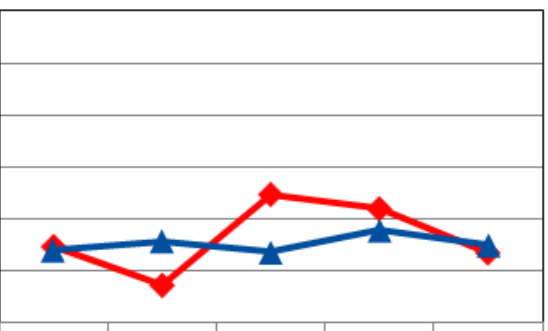

20062007200820092010

Security and Fire

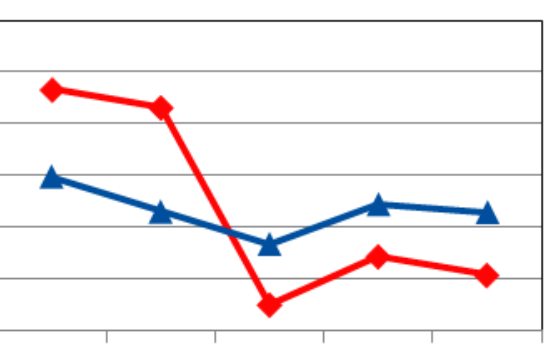

20062007200820092010

Line Operators

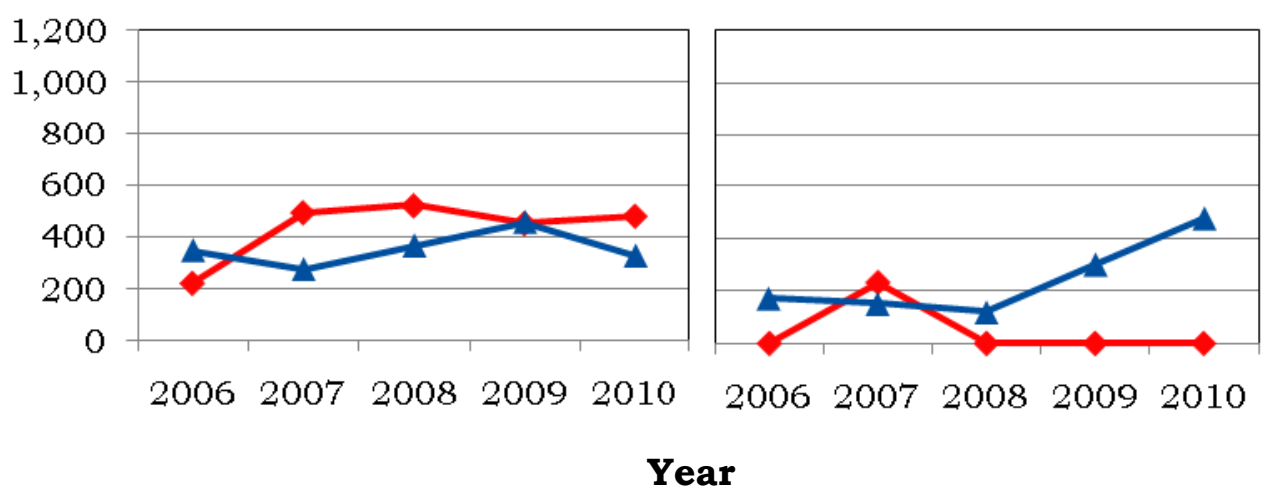

*Standardized to age distribution of 2000 U.S. population. 


\section{Sentinel Health Events for Occupations (SHEOs)}

An occupational sentinel health event (SHEO) is a disease, disability, or death that is likely to be occupationally related. Although sentinel health events may indicate an occupational exposure, many may result from nonoccupational exposures. Sentinel health events are therefore assessed in two categories:

Definite Sentinel Health Events: Diseases that are unlikely to occur in the absence of an occupational exposure (e.g., asbestosis).

Possible Sentinel Health Events: Diseases that may be occupational but can also occur in the absence of an occupational exposure (e.g., lung cancer or carpal tunnel syndrome).

Figure 16. Characteristics of SHEOs by Gender

\begin{tabular}{|l|c|c|c|c|}
\hline \multirow{2}{*}{} & \multicolumn{2}{|c|}{$\begin{array}{c}\text { Total Number of } \\
\text { SHEO Diagnoses }\end{array}$} & \multicolumn{2}{c|}{$\begin{array}{c}\text { Total Number of } \\
\text { Days Absent }\end{array}$} \\
\cline { 2 - 5 } & Men & Women & Men & W omen \\
\hline Definite & 0 & 1 & 0 & 1 \\
\hline Possible & 8 & 6 & 294 & 197 \\
\hline Total & 8 & 7 & 294 & 198 \\
\hline
\end{tabular}

Figure 17. SHEO Diagnoses by Gender

\begin{tabular}{|l|c|c|}
\hline \multirow{2}{*}{ Diagnoses } & \multicolumn{2}{c|}{ Gender } \\
\cline { 2 - 3 } & Women & M en \\
\hline Carpal Tunnel Syndrome & 3 & 6 \\
\hline Injuries & 1 & 0 \\
\hline Other Conditions & 3 & 2 \\
\hline
\end{tabular}


Occupational Safety and Health Administration (OSHA)-Recordable Events

Figure 18. OSHA-Recordable Events by Gender and Age

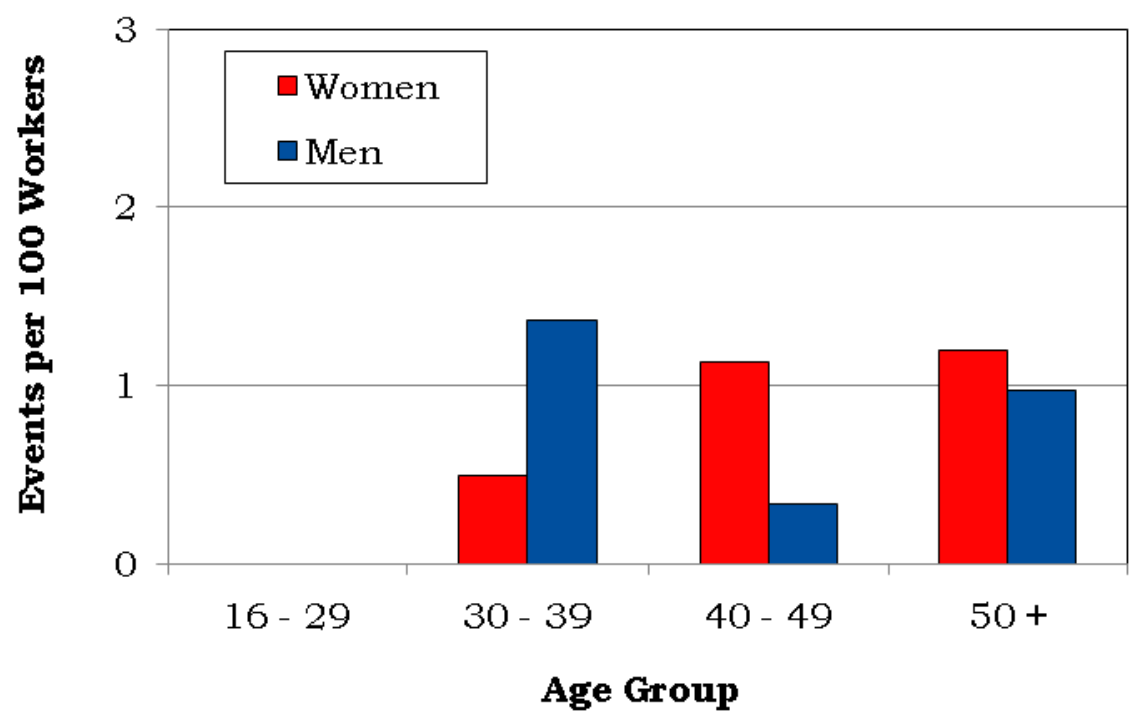

Figure 19. OSHA-Recordable Events by Job Category and Gender

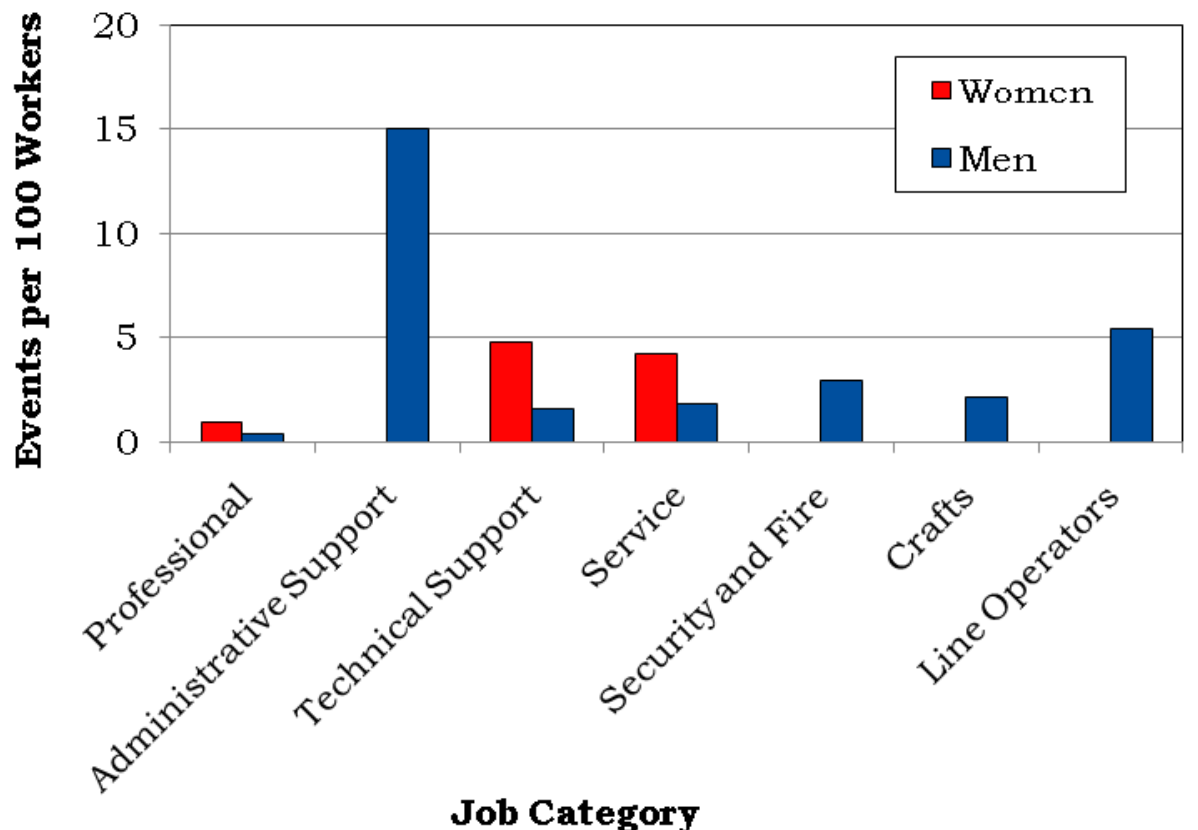

Job Category 


\section{Diagnostic and Accident Categories for OSHA-Recordable Events}

Figure 20. OSHA-Recordable Diagnoses by Diagnostic Category and Gender

\begin{tabular}{|l|c|c|}
\hline \multirow{2}{*}{ Diagnostic Category } & \multicolumn{2}{c|}{ Gender } \\
\cline { 2 - 3 } & Women & M en \\
\hline Musculoskeletal & $\mathbf{7}$ & $\mathbf{1 6}$ \\
\hline Nervous System & $\mathbf{0}$ & $\mathbf{2}$ \\
\hline Skin & $\mathbf{1}$ & $\mathbf{0}$ \\
\hline Unspecified Symptoms & $\mathbf{1}$ & $\mathbf{1}$ \\
\hline Injury & $\mathbf{1 9}$ & $\mathbf{2 9}$ \\
\hline Fractures - Upper Limb & $\mathbf{2}$ & $\mathbf{1}$ \\
\hline Fractures - Lower Limb & $\mathbf{2}$ & $\mathbf{0}$ \\
\hline Dislocations & $\mathbf{0}$ & $\mathbf{1}$ \\
\hline Back Sprains \& Strains & $\mathbf{2}$ & $\mathbf{4}$ \\
\hline Other Sprains \& Strains & $\mathbf{3}$ & $\mathbf{3}$ \\
\hline Open Wounds - Head, Neck, Trunk & $\mathbf{0}$ & $\mathbf{3}$ \\
\hline Open Wounds - Upper Limb & $\mathbf{3}$ & $\mathbf{4}$ \\
\hline Superficial Injuries & $\mathbf{2}$ & $\mathbf{1}$ \\
\hline Bruises & $\mathbf{0}$ & $\mathbf{4}$ \\
\hline Foreign Bodies Entering Orifice & $\mathbf{0}$ & $\mathbf{2}$ \\
\hline Unspecified Injuries & $\mathbf{5}$ & $\mathbf{4}$ \\
\hline Adverse Reactions to Non-Medical Substances & $\mathbf{0}$ & $\mathbf{2}$ \\
\hline
\end{tabular}


Figure 21. OSHA-Recordable Accidents by Type and Gender

\begin{tabular}{|l|c|c|}
\hline \multirow{2}{*}{ Accident Category } & \multicolumn{2}{c|}{ Gender } \\
\cline { 2 - 3 } & Women & M en \\
\cline { 2 - 3 } & $\begin{array}{c}\text { Number of } \\
\text { Accidents }\end{array}$ & $\begin{array}{c}\text { Number of } \\
\text { Accidents }\end{array}$ \\
\hline Surgical and Me dical Procedures & $\mathbf{0}$ & $\mathbf{1}$ \\
\hline Falls & $\mathbf{5}$ & $\mathbf{2}$ \\
\hline Natural/Environmental Factors & $\mathbf{0}$ & $\mathbf{2}$ \\
\hline Submersion/Suffocation/Foreign Bodies & $\mathbf{0}$ & $\mathbf{2}$ \\
\hline Other Accidents & $\mathbf{9}$ & $\mathbf{2 1}$ \\
\hline Struck by an Object & $\mathbf{1}$ & $\mathbf{8}$ \\
\hline Caught Between Objects & $\mathbf{1}$ & $\mathbf{2}$ \\
\hline Machinery & $\mathbf{0}$ & $\mathbf{1}$ \\
\hline Cutting/Piercing Instrument/Object & $\mathbf{2}$ & $\mathbf{2}$ \\
\hline Overexertion/Strenuous Movements & $\mathbf{3}$ & $\mathbf{8}$ \\
\hline Repetitive Trauma & $\mathbf{2}$ & $\mathbf{0}$ \\
\hline Total & $\mathbf{1 4}$ & $\mathbf{2 8}$ \\
\hline
\end{tabular}




\section{Rates of OSHA-Recordable Events}

Figure 22. OSHA-Recordable Rates by Age and Job Categories Among Women, All Diagnoses Combined

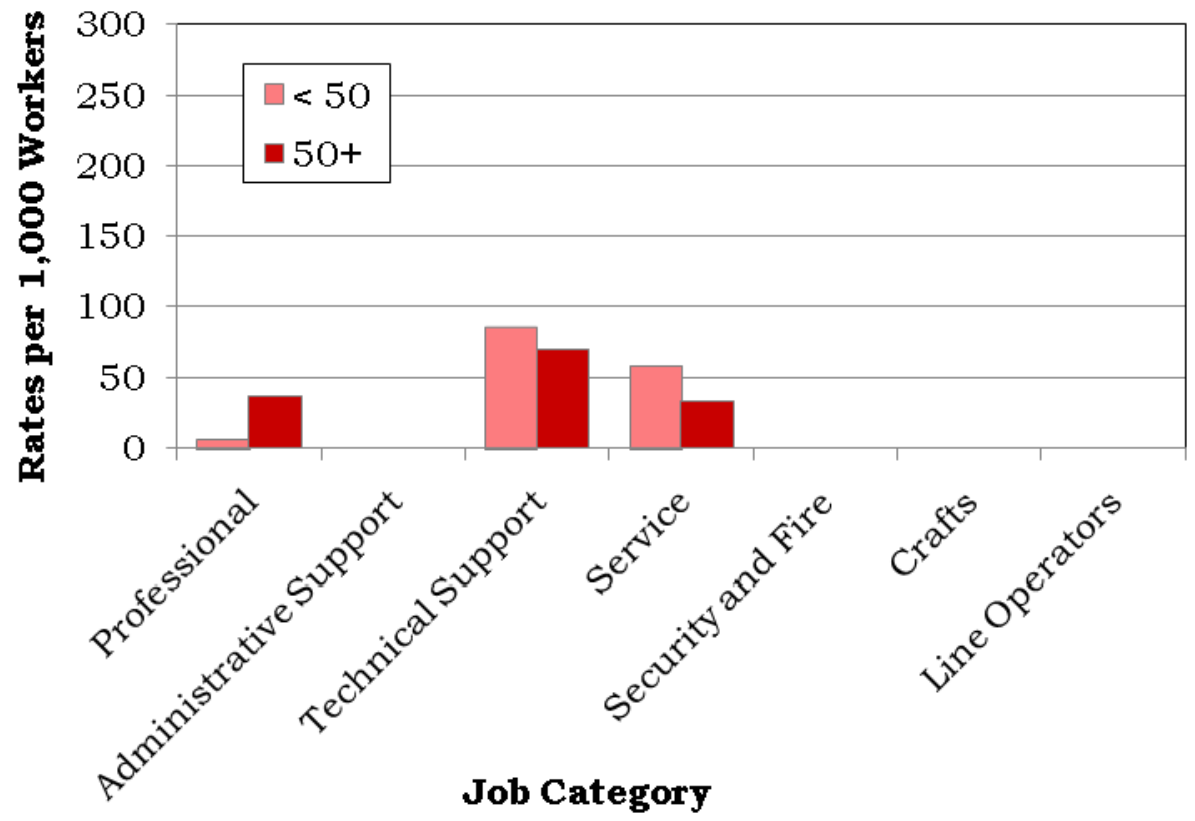

Figure 23. OSHA-Recordable Rates by Age and Job Categories Among Men, All Diagnoses Combined

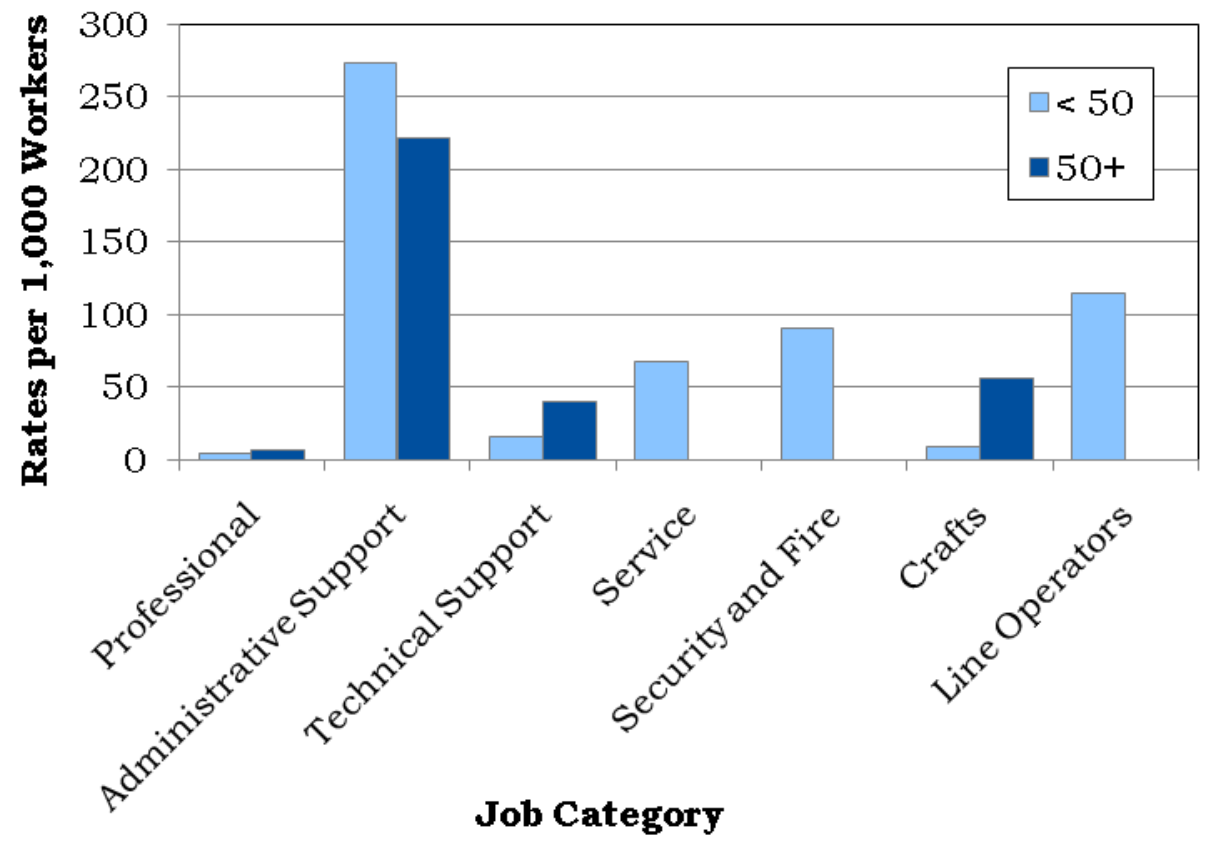




\section{Time Trends for OSHA-Recordable Events}

Figure 24. Age-Adjusted Rates for All OSHA-Recordable Diagnoses Combined Among Women and Men by Job Category from 2006 to 2010*

Professional

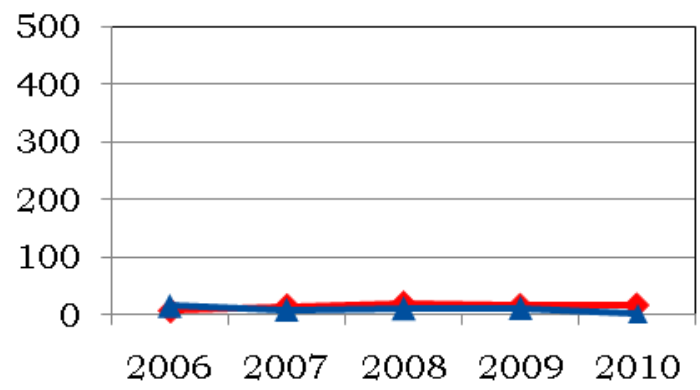

Administrative Sup port

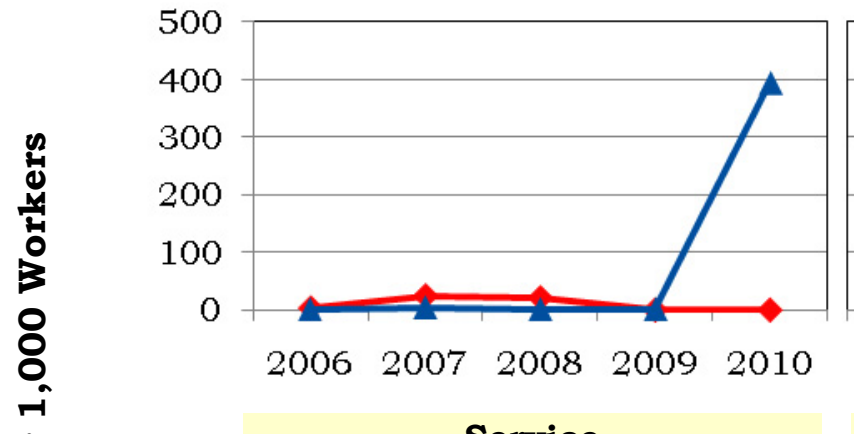

Service

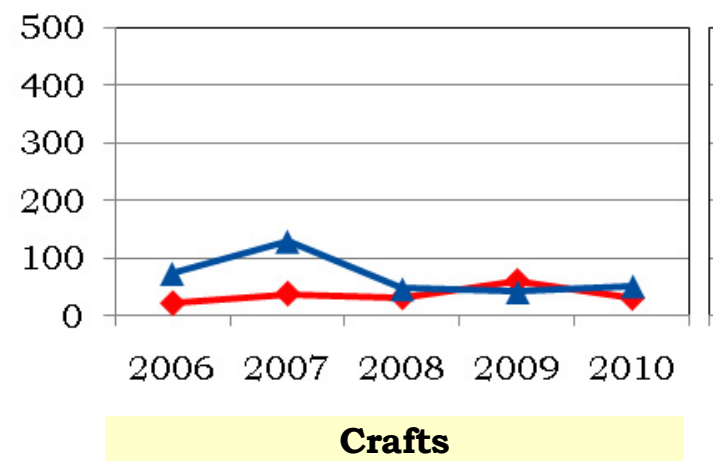

500

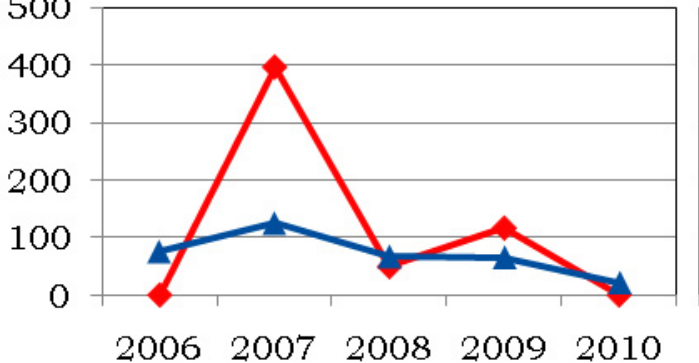

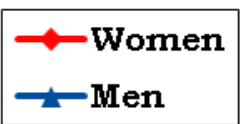

Technical Supp ort

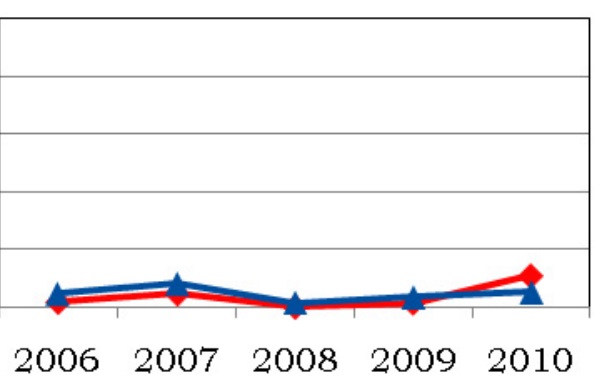

Security and Fire

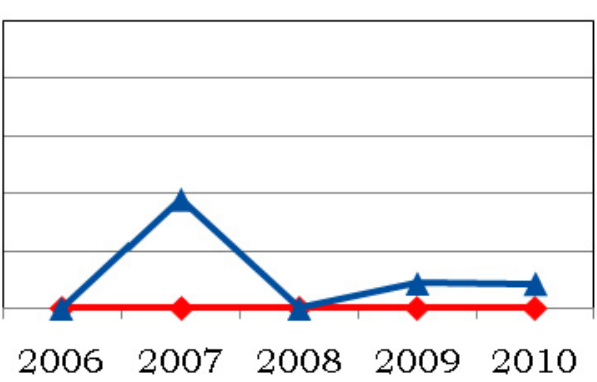

Line Op erators

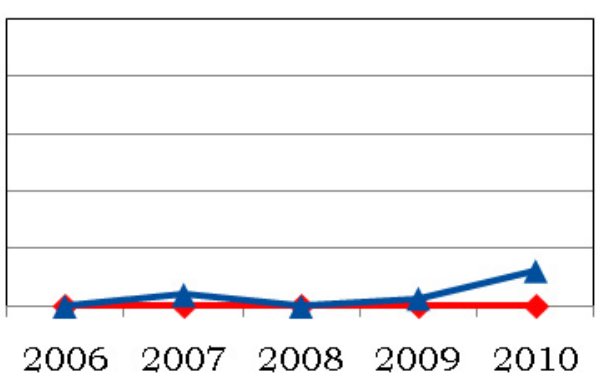

Year

*Standardized to age distribution of 2000 U.S. population. 


\section{Appendices}


Oak Ridge National Laboratory 2010

Absence Data

Appendix A. Work Force by Gender, Age, and Job Category

\begin{tabular}{|c|c|c|c|c|c|c|c|c|c|c|c|}
\hline \multirow{3}{*}{ Job Category } & \multicolumn{5}{|c|}{ Women } & \multicolumn{5}{|c|}{ Men } & \multirow[b]{3}{*}{ TOTAL } \\
\hline & \multicolumn{4}{|c|}{ Age Group } & \multirow[b]{2}{*}{ TOTAL } & \multicolumn{4}{|c|}{ Age Group } & \multirow[b]{2}{*}{ TOTAL } & \\
\hline & $16-29$ & 30 - 39 & $40-49$ & $50+$ & & $16-29$ & $30-39$ & $40-49$ & $50+$ & & \\
\hline Professional & 57 & 120 & 229 & 354 & 760 & 145 & 467 & 656 & 1,295 & 2,563 & 3,323 \\
\hline Administrative Support & 37 & 59 & 147 & 286 & 529 & 7 & 2 & 2 & 9 & 20 & 549 \\
\hline Technical Support & 9 & 20 & 40 & 57 & 126 & 23 & 63 & 100 & 125 & 311 & 437 \\
\hline Service & 2 & 4 & 11 & 30 & 47 & 30 & 21 & 23 & 35 & 109 & 156 \\
\hline Security and Fire & 0 & 2 & 3 & 4 & 9 & 2 & 11 & 9 & 12 & 34 & 43 \\
\hline Crafts & 2 & 1 & 12 & 17 & 32 & 17 & 77 & 117 & 249 & 460 & 492 \\
\hline Line Operators & 0 & 0 & 1 & 1 & 2 & 6 & 17 & 12 & 20 & 55 & 57 \\
\hline TOTAL & 107 & 206 & 443 & 749 & 1,505 & 230 & 658 & 919 & 1,745 & 3,552 & 5,057 \\
\hline
\end{tabular}

Appendix B. Age Distribution of the Work Force by Gender

\begin{tabular}{|l|r|r|r|r|r|r|r|r|}
\hline \multirow{3}{*}{ Year } & \multicolumn{4}{|c|}{ Women } & \multicolumn{4}{c|}{ Men } \\
\cline { 2 - 9 } & \multicolumn{1}{|c|}{ Percent Distribution by Age Group } & \multicolumn{1}{c|}{ Percent Distribution by Age Group } \\
\cline { 2 - 9 } & $\mathbf{1 6}-\mathbf{2 9}$ & $\mathbf{3 0}-\mathbf{3 9}$ & $\mathbf{4 0}-\mathbf{4 9}$ & $\mathbf{5 0}+$ & $\mathbf{1 6}-\mathbf{2 9}$ & $\mathbf{3 0}-\mathbf{3 9}$ & $\mathbf{4 0}-\mathbf{4 9}$ & $\mathbf{5 0}+$ \\
\hline $\mathbf{1 9 9 9}$ & 3.31 & 24.96 & 44.75 & 26.98 & 2.72 & 18.64 & 42.04 & 36.60 \\
\hline $\mathbf{2 0 0 0}$ & 3.34 & 22.32 & 44.37 & 29.97 & 2.57 & 17.20 & 40.03 & 40.20 \\
\hline $\mathbf{2 0 0 1}$ & 3.49 & 19.15 & 43.57 & 33.80 & 1.70 & 15.81 & 39.34 & 43.15 \\
\hline $\mathbf{2 0 0 2}$ & 2.16 & 19.68 & 44.02 & 34.14 & 1.82 & 15.47 & 38.48 & 44.23 \\
\hline $\mathbf{2 0 0 3}$ & 2.05 & 17.95 & 40.90 & 39.10 & 2.33 & 14.95 & 35.36 & 47.36 \\
\hline $\mathbf{2 0 0 4}$ & 2.89 & 16.85 & 39.88 & 40.38 & 2.55 & 14.57 & 35.71 & 47.16 \\
\hline $\mathbf{2 0 0 5}$ & 4.01 & 14.97 & 38.19 & 42.82 & 3.48 & 14.40 & 33.13 & 48.99 \\
\hline $\mathbf{2 0 0 6}$ & 3.69 & 14.82 & 35.14 & 46.35 & 4.39 & 14.96 & 31.32 & 49.33 \\
\hline $\mathbf{2 0 0 7}$ & 5.11 & 14.31 & 32.84 & 47.74 & 4.80 & 15.39 & 29.86 & 49.95 \\
\hline $\mathbf{2 0 0 8}$ & 5.58 & 14.55 & 31.06 & 48.81 & 5.27 & 16.75 & 28.02 & 49.95 \\
\hline $\mathbf{2 0 0 9}$ & 6.91 & 13.20 & 30.75 & 49.14 & 6.10 & 17.92 & 26.89 & 49.09 \\
\hline $\mathbf{2 0 1 0}$ & 7.11 & 13.69 & 29.44 & 49.77 & 6.48 & 18.52 & 25.87 & 49.13 \\
\hline
\end{tabular}


Oak Ridge National Laboratory 2010

Absence Data

Appendix C. Total Number of Workers Who Reported at Least One Absence by Gender, Age, and Job Category*

\begin{tabular}{|c|c|c|c|c|c|c|c|c|c|c|c|}
\hline \multirow{3}{*}{ Job Category } & \multicolumn{5}{|c|}{ Women } & \multicolumn{5}{|c|}{ Men } & \multirow[b]{3}{*}{ TOTAL } \\
\hline & \multicolumn{4}{|c|}{ Age Group } & \multirow[b]{2}{*}{ TOTAL } & \multicolumn{4}{|c|}{ Age Group } & \multirow[b]{2}{*}{ TOTAL } & \\
\hline & $16-29$ & 30 - 39 & $40-49$ & $50+$ & & $16-29$ & 30 - 39 & $40-49$ & $50+$ & & \\
\hline Professional & 8 & 13 & 30 & 59 & 110 & 10 & 35 & 51 & 153 & 249 & 359 \\
\hline Administrative Support & 3 & 19 & 38 & 83 & 143 & 0 & 0 & 1 & 2 & 3 & 146 \\
\hline Technical Support & 1 & 4 & 7 & 13 & 25 & 5 & 14 & 15 & 26 & 60 & 85 \\
\hline Service & 0 & 3 & 3 & 8 & 14 & 4 & 6 & 7 & 13 & 30 & 44 \\
\hline Security and Fire & 0 & 0 & 0 & 2 & 2 & 0 & 4 & 2 & 4 & 10 & 12 \\
\hline Crafts & 1 & 0 & 6 & 5 & 12 & 4 & 11 & 23 & 64 & 102 & 114 \\
\hline Line Operators & 0 & 0 & 0 & 0 & 0 & 0 & 1 & 4 & 8 & 13 & 13 \\
\hline TOTAL & 13 & 39 & 84 & 170 & 306 & 23 & 71 & 103 & 270 & 467 & 773 \\
\hline
\end{tabular}

*Only those job categories and gender/age combinations with at least one absence appear in this table.

Appendix D. Total Number of Absences by Gender, Age, and Job Category*

\begin{tabular}{|c|c|c|c|c|c|c|c|c|c|c|c|}
\hline \multirow{3}{*}{ Job Category } & \multicolumn{5}{|c|}{ Women } & \multicolumn{5}{|c|}{ Men } & \multirow[b]{3}{*}{ TOTAL } \\
\hline & \multicolumn{4}{|c|}{ Age Group } & \multirow[b]{2}{*}{ TOTAL } & \multicolumn{4}{|c|}{ Age Group } & \multirow[b]{2}{*}{ TOTAL } & \\
\hline & $16-29$ & 30 - 39 & $40-49$ & $50+$ & & $16-29$ & 30 - 39 & $40-49$ & $50+$ & & \\
\hline Professional & 9 & 17 & 34 & 76 & 136 & 10 & 39 & 58 & 197 & 304 & 440 \\
\hline Administrative Support & 3 & 29 & 57 & 105 & 194 & 0 & 0 & 1 & 3 & 4 & 198 \\
\hline Technical Support & 1 & 7 & 9 & 15 & 32 & 5 & 15 & 16 & 34 & 70 & 102 \\
\hline Service & 0 & 6 & 4 & 12 & 22 & 5 & 7 & 9 & 17 & 38 & 60 \\
\hline Security and Fire & 0 & 0 & 0 & 4 & 4 & 0 & 7 & 2 & 6 & 15 & 19 \\
\hline Crafts & 1 & 0 & 8 & 6 & 15 & 5 & 12 & 28 & 79 & 124 & 139 \\
\hline Line Operators & 0 & 0 & 0 & 0 & 0 & 0 & 1 & 8 & 8 & 17 & 17 \\
\hline TOTAL & 14 & 59 & 112 & 218 & 403 & 25 & 81 & 122 & 344 & 572 & 975 \\
\hline
\end{tabular}

*Only those job categories and gender/age combinations with at least one absence appear in this table. 
Oak Ridge National Laboratory 2010

Absence Data

Appendix E. Distribution of the Number of Calendar Days Missed per Absence by Gender and Age*

\begin{tabular}{|c|c|c|c|c|c|c|c|c|c|c|c|}
\hline \multirow{3}{*}{$\begin{array}{c}\text { Number of } \\
\text { Calendar Days }\end{array}$} & \multicolumn{5}{|c|}{ Women } & \multicolumn{5}{|c|}{ Men } & \multirow[b]{3}{*}{ TOTAL } \\
\hline & \multicolumn{4}{|c|}{ Age Group } & \multirow[b]{2}{*}{ TOTAL } & \multicolumn{4}{|c|}{ Age Group } & \multirow[b]{2}{*}{ TOTAL } & \\
\hline & $16-29$ & 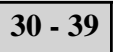 & 40 - 49 & $50+$ & & $16-29$ & $30-39$ & 40 - 49 & $50+$ & & \\
\hline$<15$ & 12 & 38 & 74 & 119 & 243 & 20 & 47 & 81 & 179 & 327 & 570 \\
\hline $15-28$ & 1 & 11 & 15 & 50 & 77 & 3 & 13 & 14 & 63 & 93 & 170 \\
\hline $29-42$ & 1 & 2 & 7 & 20 & 30 & 0 & 9 & 9 & 35 & 53 & 83 \\
\hline $43-56$ & 0 & 1 & 6 & 12 & 19 & 1 & 6 & 7 & 31 & 45 & 64 \\
\hline $57-91$ & 0 & 5 & 9 & 12 & 26 & 1 & 5 & 8 & 16 & 30 & 56 \\
\hline $92-182$ & 0 & 1 & 1 & 5 & 7 & 0 & 0 & 1 & 20 & 21 & 28 \\
\hline $183+$ & 0 & 1 & 0 & 0 & 1 & 0 & 1 & 2 & 0 & 3 & 4 \\
\hline TOTAL & 14 & 59 & 112 & 218 & 403 & 25 & 81 & 122 & 344 & 572 & 975 \\
\hline
\end{tabular}

*Only those gender/age combinations with at least one absence appear in this table. 
Oak Ridge National Laboratory 2010

Absence Data

Appendix F. Distribution of the Number of Calendar Days Missed per Absence by Gender and Job Category*

Women

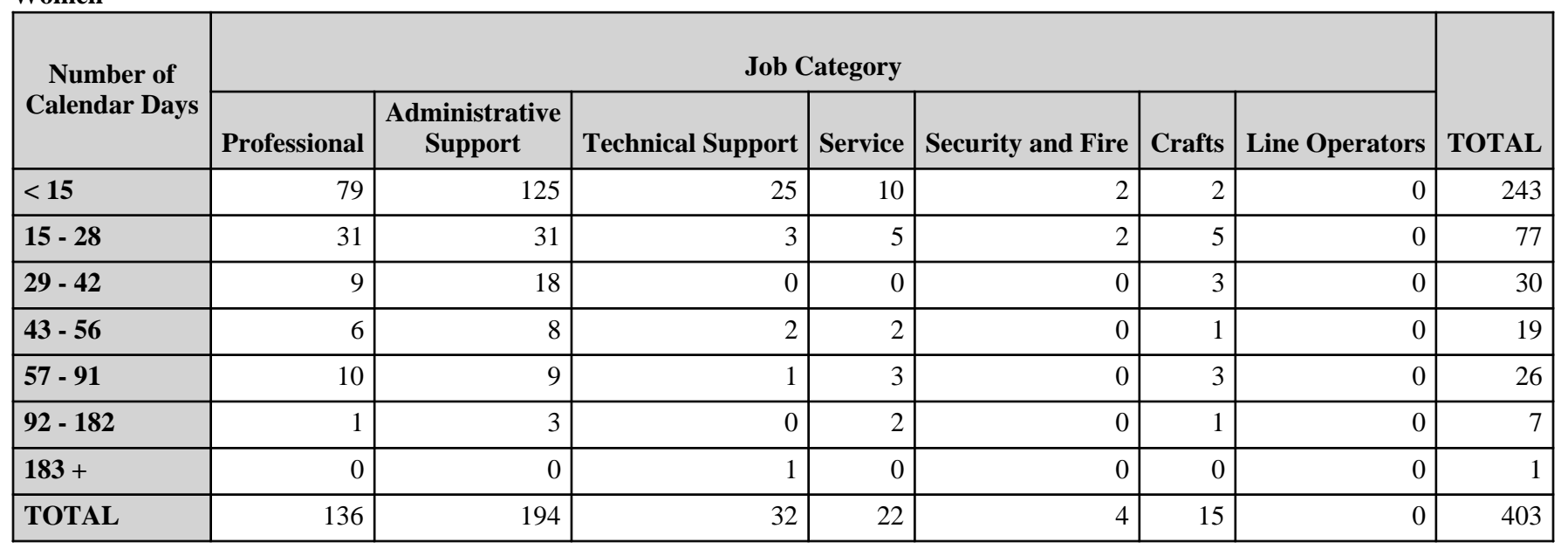

Men

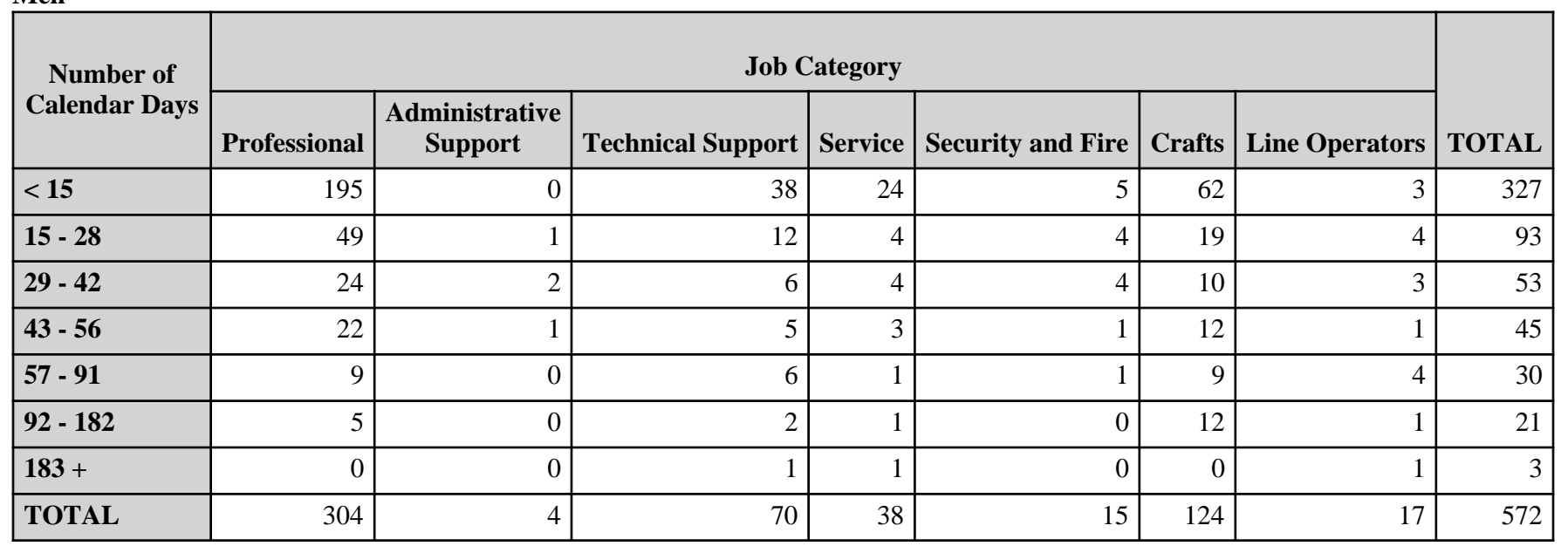

*Only those gender/job category combinations with at least one absence appear in this table. 
Oak Ridge National Laboratory 2010

Absence Data

Appendix G. Number of Diagnoses in Each Diagnostic Category by Gender and Age*

\begin{tabular}{|c|c|c|c|c|c|c|}
\hline & & \multicolumn{5}{|c|}{ Women } \\
\hline & & \multicolumn{4}{|c|}{ Age Group } & \multirow[b]{2}{*}{ TOTAL } \\
\hline & & $16-29$ & $30-39$ & $40-49$ & $50+$ & \\
\hline Diagnostic Category & ICD-9-CM Code & \multirow[b]{2}{*}{2} & \multirow[b]{2}{*}{5} & \multirow[b]{2}{*}{4} & \multirow[b]{2}{*}{8} & \multirow[b]{2}{*}{19} \\
\hline INFECTIOUS \& PARASITIC DISEASES (DIS) & 001-139 & & & & & \\
\hline -Intestinal Infectious Dis & 001-009 & 0 & 1 & 0 & 2 & 3 \\
\hline -Other Bacterial Dis & 030-041 & 1 & 3 & 1 & 2 & 7 \\
\hline -Viral Dis with Exanthem & 050-059 & 0 & 0 & 1 & 1 & 2 \\
\hline -Other Viral Dis \& Chlamydiae & 070-079 & 1 & 1 & 2 & 2 & 6 \\
\hline -Rickettsioses \& Other Arthropod & 080-088 & 0 & 0 & 0 & 1 & 1 \\
\hline MALIGNANT NEOPLASMS & $\begin{array}{l}140-208 \\
209.0-209.3 \\
230-234\end{array}$ & 0 & 1 & 4 & 8 & 13 \\
\hline -Lip, Oral Cavity, Pharynx & $140-149$ & 0 & 0 & 0 & 0 & 0 \\
\hline -Digestive \& Peritoneal & 150-159 & 0 & 0 & 1 & 0 & 1 \\
\hline -Bone, Connective Tissue, Skin & $170-173,176$ & 0 & 0 & 0 & 2 & 2 \\
\hline -Breast & 174-175 & 0 & 0 & 2 & 1 & 3 \\
\hline -Genitourinary & 179-189 & 0 & 0 & 0 & 1 & 1 \\
\hline -Endocrine & $\begin{array}{l}193-194, \\
209.0-209.3\end{array}$ & 0 & 0 & 0 & 1 & 1 \\
\hline -Other \& Unspecified Sites & 195-199, 209.7 & 0 & 0 & 1 & 3 & 4 \\
\hline -Lymphatic \& Hematopoietic & $200-208$ & 0 & 1 & 0 & 0 & 1 \\
\hline BENIGN \& UNCERTAIN NEOPLASMS & \begin{tabular}{|l|}
$209.4-209.6$ \\
$210-229$, \\
$235-239$
\end{tabular} & 0 & 0 & 6 & 5 & 11 \\
\hline ENDOCRINE/METABOLIC/IMMUNITY & $240-279$ & 0 & 0 & 1 & 6 & 7 \\
\hline -Thyroid Gland Disorders & $240-246$ & 0 & 0 & 1 & 2 & 3 \\
\hline -Other Endocrine Gland Dis & 249-259 & 0 & 0 & 0 & 0 & 0 \\
\hline -Other Metabolic \& Immunity Disorders & $270-279$ & 0 & 0 & 0 & 4 & 4 \\
\hline BLOOD \& BLOOD-FORMING ORGANS & 280-289 & 0 & 1 & 0 & 1 & 2 \\
\hline MENTAL DISORDERS & 290-319 & 0 & 4 & 1 & 4 & 9 \\
\hline -Psychoses & $290-299$ & 0 & 0 & 0 & 0 & 0 \\
\hline -Non-Psychotic Disorders & $300-302,306-316$ & 0 & 4 & 1 & 4 & 9 \\
\hline -Alcohol Dependence & 303 & 0 & 0 & 0 & 0 & 0 \\
\hline -Drug Dependence & $304-305$ & 0 & 0 & 0 & 0 & 0 \\
\hline NERVOUS SYSTEM (NS) \& SENSE ORGANS & 320-389 & 1 & 7 & 5 & 28 & 41 \\
\hline -Organic Sleep Disorders & 327 & 0 & 0 & 0 & 0 & 0 \\
\hline -Hereditary/Degenerative Central NS Dis & $330-337$ & 0 & 2 & 0 & 1 & 3 \\
\hline -Other Headache Syndromes & 339 & 0 & 0 & 0 & 0 & 0 \\
\hline -Other Disorders of Central NS & $340-349$ & 1 & 0 & 0 & 3 & $\overline{4}$ \\
\hline -Disorders of Peripheral NS & $350-359$ & 0 & 1 & 1 & 6 & 8 \\
\hline -Disorders of Eye & $360-379$ & 0 & 0 & 1 & 11 & 12 \\
\hline -Diseases of Ear \& Mastoid & 380-389 & 0 & 4 & 3 & 7 & 14 \\
\hline
\end{tabular}

(Continued)

*Only those diagnostic categories and gender/age combinations with at least one occurrence appear in this table. 
Oak Ridge National Laboratory 2010

Absence Data

Appendix G. Number of Diagnoses in Each Diagnostic Category by Gender and Age*

\begin{tabular}{|c|c|c|c|c|c|c|c|}
\hline & & \multicolumn{5}{|c|}{ Men } & \multirow[b]{3}{*}{ TOTAL } \\
\hline & & \multicolumn{4}{|c|}{ Age Group } & \multirow[b]{2}{*}{ TOTAL } & \\
\hline & & $16-29$ & $30-39$ & $40-49$ & $50+$ & & \\
\hline INFECTIOUS \& PARASITIC DISEASES (DIS) & 001-139 & 4 & 4 & 4 & 14 & 26 & 45 \\
\hline -Intestinal Infectious Dis & 001-009 & 1 & 0 & 0 & 0 & 1 & 4 \\
\hline -Viral Dis with Exanthem & $050-059$ & 0 & 0 & 1 & 2 & 3 & 5 \\
\hline -Other Viral Dis \& Chlamydiae & 070-079 & 2 & 1 & 2 & 6 & 11 & 17 \\
\hline -Rickettsioses \& Other Arthropod & 080-088 & 0 & 0 & 0 & 0 & 0 & 1 \\
\hline MALIGNANT NEOPLASMS & $\begin{array}{l}140-208, \\
209.0-209.3 \\
230-234\end{array}$ & 0 & 0 & 0 & 7 & 7 & 20 \\
\hline -Digestive \& Peritoneal & $150-159$ & 0 & 0 & 0 & 0 & 0 & 1 \\
\hline -Bone, Connective Tissue, Skin & $170-173,176$ & 0 & 0 & 0 & 0 & 0 & 2 \\
\hline -Breast & $174-175$ & 0 & 0 & 0 & 0 & 0 & 3 \\
\hline -Genitourinary & 179-189 & 0 & 0 & 0 & 6 & 6 & 7 \\
\hline -Endocrine & \begin{tabular}{|l}
$193-194$ \\
$209.0-209.3$
\end{tabular} & 0 & 0 & 0 & 0 & 0 & 1 \\
\hline -Other \& Unspecified Sites & 195-199, 209.7 & 0 & 0 & 0 & 0 & 0 & 4 \\
\hline -Lymphatic \& Hematopoietic & $200-208$ & 0 & 0 & 0 & 0 & 0 & 1 \\
\hline BENIGN \& UNCERTAIN NEOPLASMS & $\begin{array}{l}209.4-209.6 \\
210-229,235-239\end{array}$ & 0 & 1 & 3 & 12 & 16 & 27 \\
\hline MENTAL DISORDERS & 290-319 & 0 & 2 & 7 & 5 & 14 & 23 \\
\hline -Psychoses & 290-299 & 0 & 0 & 1 & 0 & 1 & 1 \\
\hline -Non-Psychotic Disorders & $300-302,306-316$ & 0 & 0 & 2 & 5 & 7 & 16 \\
\hline -Alcohol Dependence & 303 & 0 & 0 & 2 & 0 & 2 & 2 \\
\hline -Drug Dependence & 304-305 & 0 & 2 & 2 & 0 & 4 & 4 \\
\hline NERVOUS SYSTEM (NS) \& SENSE ORGANS & $320-389$ & 0 & 4 & 10 & 35 & 49 & 90 \\
\hline -Organic Sleep Disorders & 327 & 0 & 2 & 1 & 0 & 3 & 3 \\
\hline -Hereditary/Degenerative Central NS Dis & 330-337 & 0 & 0 & 0 & 1 & 1 & 4 \\
\hline -Other Headache Syndromes & 339 & 0 & 0 & 0 & 1 & 1 & 1 \\
\hline -Other Disorders of Central NS & $340-349$ & 0 & 0 & 2 & 0 & 2 & 6 \\
\hline -Disorders of Peripheral NS & 350-359 & 0 & 1 & 2 & 11 & 14 & 22 \\
\hline -Disorders of Eye & $360-379$ & 0 & 0 & 2 & 12 & 14 & 26 \\
\hline -Diseases of Ear \& Mastoid & $380-389$ & 0 & 1 & 3 & 10 & 14 & 28 \\
\hline
\end{tabular}

*Only those diagnostic categories and gender/age combinations with at least one occurrence appear in this table. 
Oak Ridge National Laboratory 2010

Absence Data

Appendix G. Number of Diagnoses in Each Diagnostic Category by Gender and Age*

\begin{tabular}{|c|c|c|c|c|c|c|}
\hline & & \multicolumn{5}{|c|}{ Women } \\
\hline & & \multicolumn{4}{|c|}{ Age Group } & \multirow[b]{2}{*}{ TOTAL } \\
\hline & & $16-29$ & $30-39$ & $40-49$ & $50+$ & \\
\hline Diagnostic Category & ICD-9-CM Code & \multirow[b]{2}{*}{0} & \multirow[b]{2}{*}{1} & \multirow[b]{2}{*}{1} & \multirow[b]{2}{*}{11} & \multirow[b]{2}{*}{13} \\
\hline CIRCULATORY SYSTEM & $390-459$ & & & & & \\
\hline -Hypertensive Dis & $401-405$ & 0 & 1 & 0 & 2 & 3 \\
\hline -Ischemic Heart Dis & $410-414$ & 0 & 0 & 0 & 4 & 4 \\
\hline -Dis of Pulmonary Circulation & 415-417 & 0 & 0 & 0 & 0 & 0 \\
\hline -Other Heart Dis & $420-429$ & 0 & 0 & 0 & 2 & 2 \\
\hline -Cerebrovascular Dis & $430-438$ & 0 & 0 & 1 & 2 & 3 \\
\hline -Dis of Arteries \& Capillaries & $440-449$ & 0 & 0 & 0 & 0 & 0 \\
\hline -Dis of Veins, Lymphatics, Other & 451-459 & 0 & 0 & 0 & 1 & 1 \\
\hline RESPIRATORY SYSTEM & $460-519$ & 7 & 23 & 33 & 58 & 121 \\
\hline -Acute Respiratory Infections & $460-466$ & 4 & 6 & 5 & 22 & 37 \\
\hline -Other Dis Upper Respiratory Tract & $470-478$ & 2 & 11 & 18 & 15 & 46 \\
\hline -Pneumonia \& Influenza & $480-488$ & 0 & 4 & 5 & 8 & 17 \\
\hline -Chronic Obstructive Dis & $490-496$ & 1 & 2 & 5 & 13 & 21 \\
\hline -Other Respiratory Dis & $510-519$ & 0 & 0 & 0 & 0 & 0 \\
\hline DIGESTIVE SYSTEM & $520-579$ & 3 & 12 & 25 & 33 & 73 \\
\hline -Oral Cavity, Saliva Glands, Jaw & $520-529$ & 2 & 0 & 4 & 6 & 12 \\
\hline -Esophagus, Stomach, Duodenum & $530-538$ & 0 & 2 & 0 & 4 & 6 \\
\hline -Appendicitis & $540-543$ & 0 & 0 & 0 & 1 & 1 \\
\hline -Hernias & $550-553$ & 0 & 0 & 1 & 2 & 3 \\
\hline -Enteritis, Colitis & $555-558$ & 1 & 5 & 11 & 10 & 27 \\
\hline -Other Intestinal Dis & $560-569$ & 0 & 3 & 4 & 5 & 12 \\
\hline -Other Digestive Dis & 570-579 & 0 & 2 & 5 & 5 & 12 \\
\hline GENITOURINARY SYSTEM & $580-629$ & 0 & 10 & 16 & 14 & 40 \\
\hline -Nephritis, Nephrosis & $580-589$ & 0 & 0 & 0 & 1 & 1 \\
\hline -Other Urinary Dis & $590-599$ & 0 & 1 & 2 & 2 & 5 \\
\hline -Male Genital Organ Dis & $600-608$ & 0 & 0 & 0 & 0 & 0 \\
\hline -Breast Disorders & $610-612$ & 0 & 2 & 1 & 2 & 5 \\
\hline -Pelvic Inflammatory Dis & 614-616 & 0 & 2 & 1 & 1 & 4 \\
\hline -Other Female Disorders & $617-629$ & 0 & 5 & 12 & 8 & 25 \\
\hline SKIN AND SUBCUTANEOUS TISSUE & 680-709 & 0 & 0 & 5 & 3 & 8 \\
\hline -Infections & $680-686$ & 0 & 0 & 1 & 1 & 2 \\
\hline -Other Inflammatory Conditions & 690-698 & 0 & 0 & 2 & 0 & 2 \\
\hline -Other & 700-709 & 0 & 0 & 2 & 2 & 4 \\
\hline $\begin{array}{l}\text { MUSCULOSKELETAL \& CONNECTIVE } \\
\text { TISSUE }\end{array}$ & $710-739$ & 1 & 11 & 19 & 55 & 86 \\
\hline -Arthropathies & $710-719$ & 0 & 4 & 7 & 23 & 34 \\
\hline -Dorsopathies & $720-724$ & 1 & 6 & 5 & 20 & 32 \\
\hline
\end{tabular}

(Continued)

*Only those diagnostic categories and gender/age combinations with at least one occurrence appear in this table. 
Oak Ridge National Laboratory 2010

Absence Data

Appendix G. Number of Diagnoses in Each Diagnostic Category by Gender and Age*

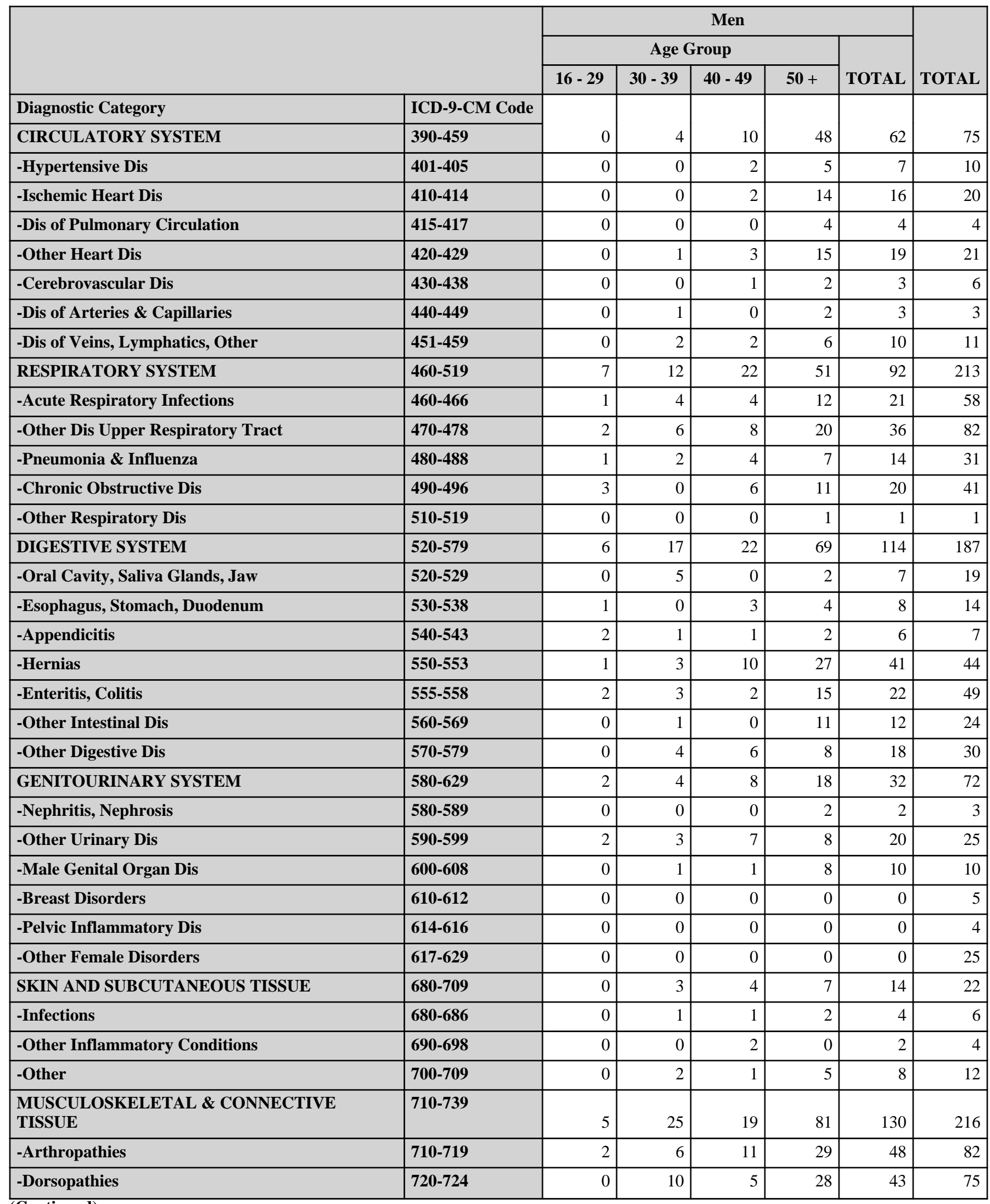

(Continued)

*Only those diagnostic categories and gender/age combinations with at least one occurrence appear in this table. 
Oak Ridge National Laboratory 2010

Absence Data

Appendix G. Number of Diagnoses in Each Diagnostic Category by Gender and Age*

\begin{tabular}{|c|c|c|c|c|c|c|}
\hline & & \multicolumn{5}{|c|}{ Women } \\
\hline & & \multicolumn{4}{|c|}{ Age Group } & \multirow[b]{2}{*}{ TOTAL } \\
\hline & & $16-29$ & $30-39$ & $40-49$ & $50+$ & \\
\hline Diagnostic Category & ICD-9-CM Code & \multirow[b]{2}{*}{0} & \multirow[b]{2}{*}{1} & \multirow[b]{2}{*}{4} & \multirow[b]{2}{*}{10} & \multirow[b]{2}{*}{15} \\
\hline -Rheumatism, Excluding Back & $725-729$ & & & & & \\
\hline -Other Dis \& Acquired Deformities & 730-739 & 0 & 0 & 3 & 2 & 5 \\
\hline CONGENITAL ANOMALIES & $740-759$ & 0 & 1 & 0 & 0 & 1 \\
\hline $\begin{array}{l}\text { SYMPTOMS, SIGNS, \& ILL-DEFINED } \\
\text { CONDITIONS }\end{array}$ & $780-799$ & 0 & 10 & 5 & 18 & 33 \\
\hline -Symptoms & 780-789 & 0 & 9 & 5 & 16 & 30 \\
\hline -Non-Specific Abnormal Findings & $790-796$ & 0 & 1 & 0 & 2 & 3 \\
\hline INJURY \& POISONING & $800-999$ & 5 & 6 & 16 & 40 & 67 \\
\hline -Fracture - Skull & $800-804$ & 3 & 1 & 0 & 0 & 4 \\
\hline -Fracture - Neck, Trunk & $805-809$ & 0 & 1 & 0 & 2 & 3 \\
\hline -Fracture - Upper Limb & $810-819$ & 0 & 0 & 2 & 2 & 4 \\
\hline -Fracture - Lower Limb & $820-829$ & 0 & 1 & 2 & 6 & 9 \\
\hline -Dislocation & 830-839 & 0 & 0 & 1 & 11 & 12 \\
\hline -Sprains \& Strains - Back & 846-847 & 1 & 0 & 4 & 1 & 6 \\
\hline -Sprains \& Strains - Other & $840-845,848$ & 0 & 2 & 3 & 8 & 13 \\
\hline -Intracranial Injury & $850-854$ & 0 & 0 & 0 & 0 & 0 \\
\hline -Internal Injury - Thorax, Abdomen, Pelvis & $860-869$ & 0 & 0 & 0 & 0 & 0 \\
\hline -Open Wound - Head, Neck, Trunk & $870-879$ & 1 & 0 & 1 & 0 & 2 \\
\hline -Open Wound - Upper Limb & $880-887$ & 0 & 0 & 0 & 0 & 0 \\
\hline -Open Wound - Lower Limb & $890-897$ & 0 & 0 & 0 & 1 & 1 \\
\hline -Late Effects of Accident & 905-909 & 0 & 0 & 0 & 1 & 1 \\
\hline -Superficial Injury & 910-919 & 0 & 0 & 0 & 0 & 0 \\
\hline -Contusion & $920-924$ & 0 & 0 & 0 & 4 & 4 \\
\hline -Burns & 940-949 & 0 & 0 & 0 & 0 & 0 \\
\hline -Injury to Nerves \& Spinal Cord & $950-957$ & 0 & 0 & 0 & 0 & 0 \\
\hline -Unspecified Effects - External Causes & $990-995$ & 0 & 0 & 1 & 0 & 1 \\
\hline -Complications of Surgical/Medical Care & 996-999 & 0 & 1 & 2 & 4 & 7 \\
\hline $\begin{array}{l}\text { HEALTH STATUS/HEALTH SERVICE } \\
\text { CONTACT }\end{array}$ & V01-V89 & 0 & 1 & 3 & 6 & 10 \\
\hline -Personal \& Family History & V10-V19 & 0 & 0 & 1 & 0 & 1 \\
\hline -Health Services Reproduction/Development & V20-V29 & 0 & 1 & 0 & 0 & 1 \\
\hline -Health Status & V40-V49 & 0 & 0 & 0 & 2 & 2 \\
\hline -Specific Procedures/Aftercare & V50-V59 & 0 & 0 & 2 & 4 & 6 \\
\hline -Examination \& Investigation & V70-V82 & 0 & 0 & 0 & 0 & 0 \\
\hline
\end{tabular}

*Only those diagnostic categories and gender/age combinations with at least one occurrence appear in this table. 
Oak Ridge National Laboratory 2010

Absence Data

Appendix G. Number of Diagnoses in Each Diagnostic Category by Gender and Age*

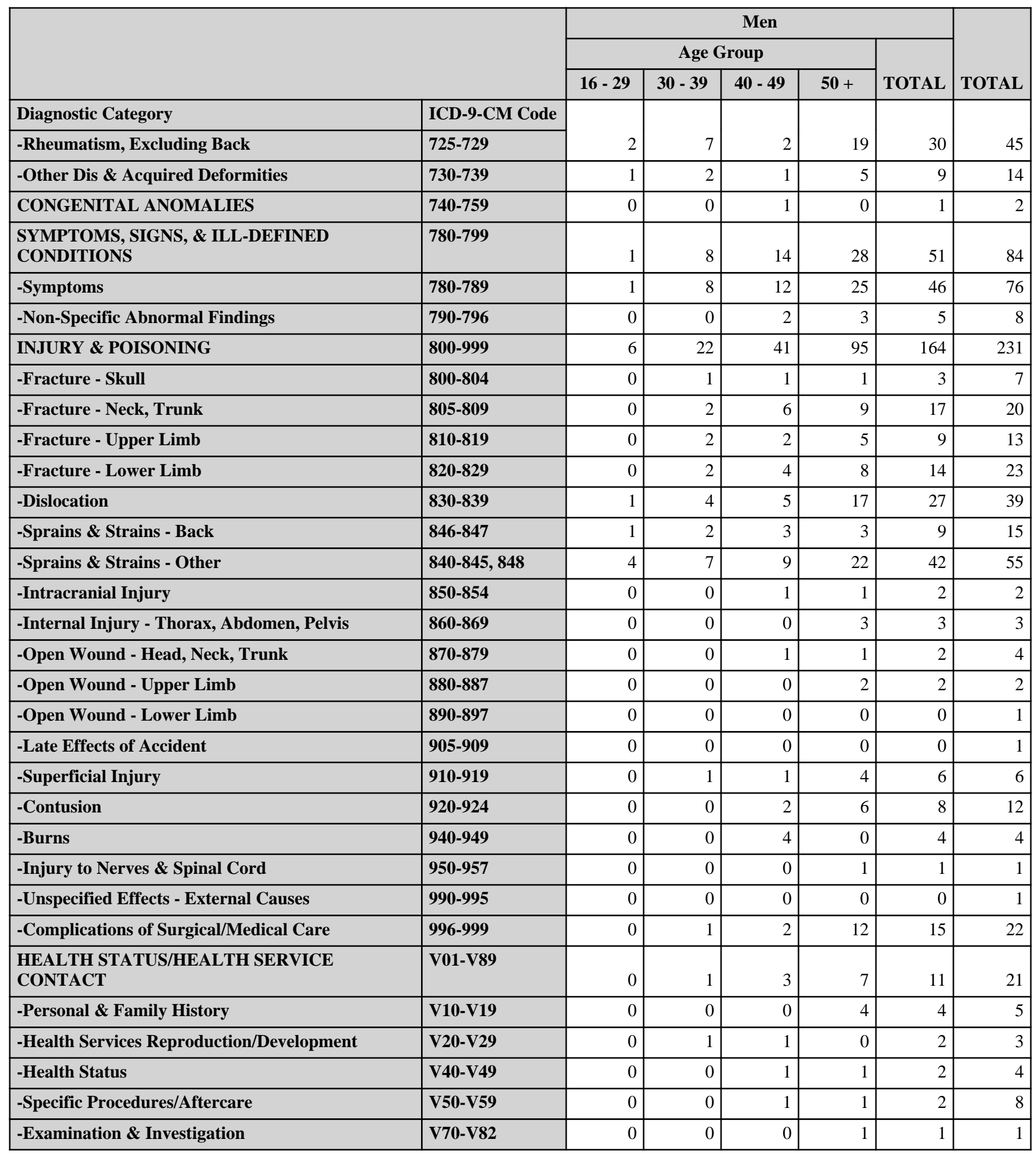

*Only those diagnostic categories and gender/age combinations with at least one occurrence appear in this table. 
Oak Ridge National Laboratory 2010

Absence Data

Appendix G. Number of Diagnoses in Each Diagnostic Category by Gender and Age*

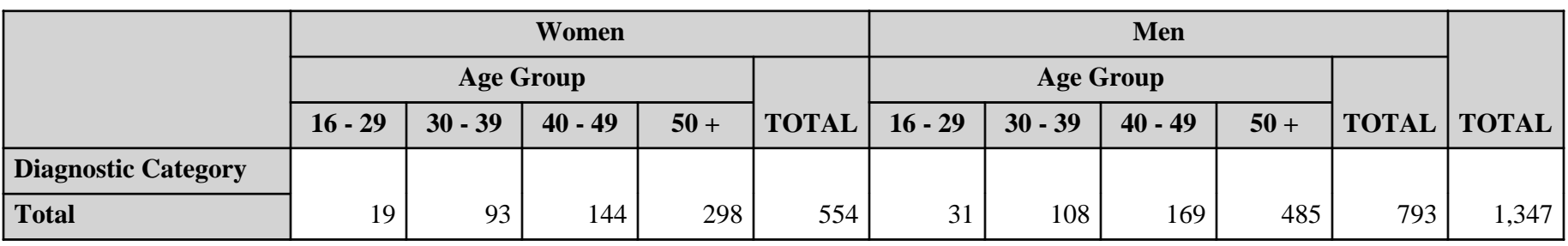

*Only those diagnostic categories and gender/age combinations with at least one occurrence appear in this table. 
Oak Ridge National Laboratory 2010

Absence Data

Appendix H. Total Number of Calendar Days Absent in Each Diagnostic Category by Gender and Age*

\begin{tabular}{|c|c|c|c|c|c|c|}
\hline & & \multicolumn{5}{|c|}{ Women } \\
\hline & & \multicolumn{4}{|c|}{ Age Group } & \multirow[b]{2}{*}{ TOTAL } \\
\hline & & $16-29$ & $30-39$ & $40-49$ & $\mathbf{5 0 +}$ & \\
\hline Diagnostic Category & ICD-9-CM Code & \multirow[b]{2}{*}{17} & \multirow[b]{2}{*}{39} & \multirow[b]{2}{*}{26} & \multirow[b]{2}{*}{154} & \multirow[b]{2}{*}{236} \\
\hline INFECTIOUS \& PARASITIC DISEASES (DIS) & 001-139 & & & & & \\
\hline MALIGNANT NEOPLASMS & $\begin{array}{l}140-208 \\
209.0-209.3 \\
230-234\end{array}$ & 0 & 2 & 121 & 122 & 245 \\
\hline BENIGN \& UNCERTAIN NEOPLASMS & $\begin{array}{l}209.4-209.6 \\
210-229,235-239\end{array}$ & 0 & 0 & 158 & 85 & 243 \\
\hline ENDOCRINE/METABOLIC/IMMUNITY & $240-279$ & 0 & 0 & 7 & 94 & 101 \\
\hline BLOOD \& BLOOD-FORMING ORGANS & $280-289$ & 0 & 13 & 0 & 6 & 19 \\
\hline MENTAL DISORDERS & 290-319 & 0 & 373 & 84 & 72 & 529 \\
\hline NERVOUS SYSTEM (NS) \& SENSE ORGANS & $320-389$ & 8 & 141 & 92 & 517 & 758 \\
\hline CIRCULATORY SYSTEM & $390-459$ & 0 & 10 & 31 & 283 & 324 \\
\hline RESPIRATORY SYSTEM & $460-519$ & 51 & 151 & 206 & 531 & 939 \\
\hline DIGESTIVE SYSTEM & $520-579$ & 51 & 250 & 212 & 432 & 945 \\
\hline GENITOURINARY SYSTEM & $580-629$ & 0 & 190 & 412 & 292 & 894 \\
\hline SKIN AND SUBCUTANEOUS TISSUE & 680-709 & 0 & 0 & 62 & 153 & 215 \\
\hline $\begin{array}{l}\text { MUSCULOSKELETAL \& CONNECTIVE } \\
\text { TISSUE }\end{array}$ & 710-739 & 9 & 274 & 682 & 2,066 & 3,031 \\
\hline CONGENITAL ANOMALIES & 740-759 & 0 & 22 & 0 & 0 & 22 \\
\hline $\begin{array}{l}\text { SYMPTOMS, SIGNS, \& ILL-DEFINED } \\
\text { CONDITIONS }\end{array}$ & $780-799$ & 0 & 110 & 46 & 189 & 345 \\
\hline INJURY \& POISONING & $800-999$ & 44 & 203 & 407 & 970 & 1,624 \\
\hline
\end{tabular}

\footnotetext{
*Absences with >1 ICD-9-CM code in the same diagnostic category were counted only once. Only those diagnostic categories and gender/age combinations with at least one occurrence appear in this table.
} 
Oak Ridge National Laboratory 2010

Absence Data

Appendix H. Total Number of Calendar Days Absent in Each Diagnostic Category by Gender and Age*

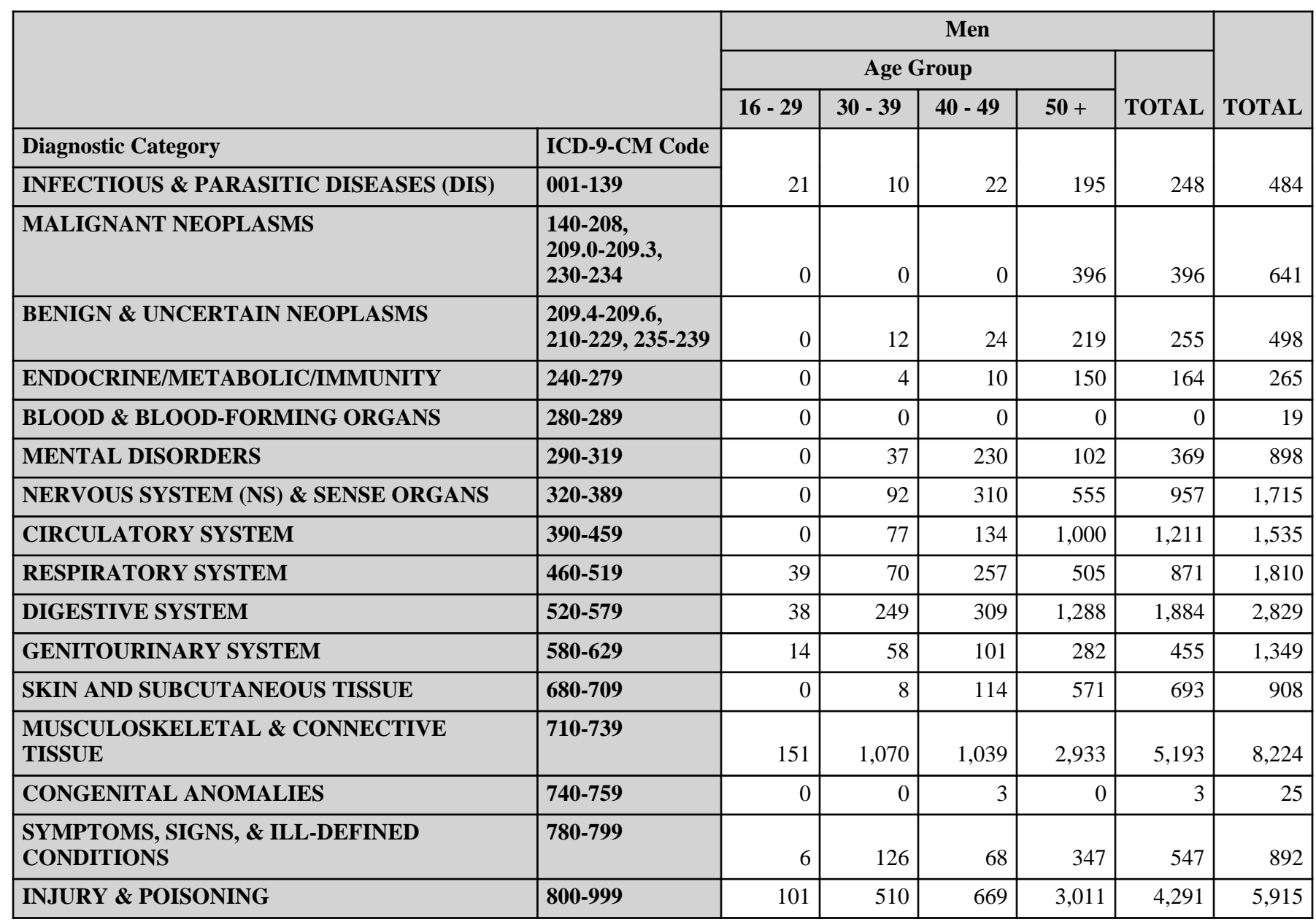

\footnotetext{
*Absences with >1 ICD-9-CM code in the same diagnostic category were counted only once. Only those diagnostic categories and gender/age combinations with at least one occurrence appear in this table.
} 
Oak Ridge National Laboratory 2010

Absence Data

Appendix I. Number of Diagnoses in Each Diagnostic Category by Gender and Job Category*

\begin{tabular}{|c|c|c|c|c|}
\hline & \multicolumn{3}{|c|}{ Women } \\
\hline & & \multicolumn{3}{|c|}{ Job Category } \\
\hline & & Professional & $\begin{array}{c}\text { Administrative } \\
\text { Support }\end{array}$ & Technical Support \\
\hline Diagnostic Category & ICD-9-CM Code & \multirow[b]{2}{*}{9} & \multirow[b]{2}{*}{7} & \multirow[b]{2}{*}{1} \\
\hline INFECTIOUS \& PARASITIC DISEASES (DIS) & 001-139 & & & \\
\hline -Intestinal Infectious Dis & 001-009 & 2 & 1 & 0 \\
\hline -Other Bacterial Dis & 030-041 & 3 & 2 & 0 \\
\hline -Viral Dis with Exanthem & 050-059 & 1 & 1 & 0 \\
\hline -Other Viral Dis \& Chlamydiae & 070-079 & 2 & 3 & 1 \\
\hline -Rickettsioses \& Other Arthropod & $080-088$ & 1 & 0 & 0 \\
\hline MALIGNANT NEOPLASMS & $\begin{array}{l}140-208 \\
209.0-209.3 \\
230-234\end{array}$ & 10 & 2 & 1 \\
\hline -Digestive \& Peritoneal & $150-159$ & 1 & 0 & 0 \\
\hline -Bone, Connective Tissue, Skin & 170-173, 176 & 2 & 0 & 0 \\
\hline -Breast & 174-175 & 2 & 1 & 0 \\
\hline -Genitourinary & $179-189$ & 0 & 0 & 1 \\
\hline -Endocrine & \begin{tabular}{|l|} 
193-194, \\
$209.0-209.3$
\end{tabular} & 1 & 0 & 0 \\
\hline -Other \& Unspecified Sites & 195-199, 209.7 & 4 & 0 & 0 \\
\hline -Lymphatic \& Hematopoietic & $200-208$ & 0 & 1 & $\overline{0}$ \\
\hline BENIGN \& UNCERTAIN NEOPLASMS & \begin{tabular}{|l|}
$209.4-209.6$ \\
$210-229$, \\
$235-239$
\end{tabular} & 3 & 8 & 0 \\
\hline ENDOCRINE/METABOLIC/IMMUNITY & $240-279$ & 2 & 4 & 0 \\
\hline -Thyroid Gland Disorders & $240-246$ & 1 & 1 & 0 \\
\hline -Other Metabolic \& Immunity Disorders & $270-279$ & 1 & 3 & 0 \\
\hline BLOOD \& BLOOD-FORMING ORGANS & $280-289$ & 0 & 2 & 0 \\
\hline MENTAL DISORDERS & $290-319$ & 3 & 2 & 2 \\
\hline -Non-Psychotic Disorders & $300-302,306-316$ & 3 & 2 & 2 \\
\hline NERVOUS SYSTEM (NS) \& SENSE ORGANS & 320-389 & 16 & 18 & 4 \\
\hline -Hereditary/Degenerative Central NS Dis & $330-337$ & 2 & 0 & 1 \\
\hline -Other Disorders of Central NS & $340-349$ & 2 & 2 & 0 \\
\hline -Disorders of Peripheral NS & $350-359$ & 5 & 1 & 2 \\
\hline -Disorders of Eye & $360-379$ & 5 & 4 & 1 \\
\hline -Diseases of Ear \& Mastoid & $380-389$ & 2 & 11 & 0 \\
\hline CIRCULATORY SYSTEM & $390-459$ & 5 & 7 & 0 \\
\hline -Hypertensive Dis & $401-405$ & 1 & 1 & 0 \\
\hline -Ischemic Heart Dis & $410-414$ & 2 & 2 & 0 \\
\hline -Other Heart Dis & $420-429$ & 0 & 2 & 0 \\
\hline -Cerebrovascular Dis & $430-438$ & 1 & 2 & 0 \\
\hline -Dis of Veins, Lymphatics, Other & 451-459 & 1 & 0 & 0 \\
\hline
\end{tabular}

(Continued)

*Only those diagnostic categories and gender/job category combinations with at least one occurrence appear in this table. 
Oak Ridge National Laboratory 2010

Absence Data

Appendix I. Number of Diagnoses in Each Diagnostic Category by Gender and Job Category*

\begin{tabular}{|c|c|c|c|c|c|}
\hline & & \multicolumn{4}{|c|}{ Women } \\
\hline & & \multicolumn{3}{|c|}{ Job Category } & \multirow[b]{2}{*}{ TOTAL } \\
\hline & & Service & Security and Fire & Crafts & \\
\hline Diagnostic Category & ICD-9-CM Code & \multirow[b]{2}{*}{2} & \multirow[b]{2}{*}{0} & \multirow[b]{2}{*}{0} & \multirow[b]{2}{*}{19} \\
\hline INFECTIOUS \& PARASITIC DISEASES (DIS) & 001-139 & & & & \\
\hline -Intestinal Infectious Dis & 001-009 & 0 & 0 & 0 & 3 \\
\hline -Other Bacterial Dis & 030-041 & 2 & 0 & 0 & 7 \\
\hline -Viral Dis with Exanthem & $050-059$ & 0 & 0 & 0 & 2 \\
\hline -Other Viral Dis \& Chlamydiae & 070-079 & 0 & 0 & 0 & 6 \\
\hline -Rickettsioses \& Other Arthropod & $080-088$ & 0 & 0 & 0 & 1 \\
\hline MALIGNANT NEOPLASMS & $\begin{array}{l}140-208, \\
209.0-209.3 \\
230-234\end{array}$ & 0 & 0 & 0 & 13 \\
\hline -Digestive \& Peritoneal & $150-159$ & 0 & 0 & 0 & 1 \\
\hline -Bone, Connective Tissue, Skin & 170-173, 176 & 0 & 0 & 0 & 2 \\
\hline -Breast & $174-175$ & 0 & 0 & 0 & 3 \\
\hline -Genitourinary & 179-189 & 0 & 0 & 0 & 1 \\
\hline -Endocrine & $\begin{array}{l}\text { 193-194, } \\
\text { 209.0-209.3 }\end{array}$ & 0 & 0 & 0 & 1 \\
\hline -Other \& Unspecified Sites & 195-199, 209.7 & 0 & 0 & 0 & 4 \\
\hline -Lymphatic \& Hematopoietic & $200-208$ & 0 & 0 & 0 & 1 \\
\hline BENIGN \& UNCERTAIN NEOPLASMS & $\begin{array}{l}209.4-209.6 \\
210-229,235-239\end{array}$ & 0 & 0 & 0 & 11 \\
\hline ENDOCRINE/METABOLIC/IMMUNITY & $240-279$ & 0 & 1 & 0 & 7 \\
\hline -Thyroid Gland Disorders & $240-246$ & 0 & 1 & 0 & 3 \\
\hline -Other Metabolic \& Immunity Disorders & 270-279 & 0 & 0 & 0 & 4 \\
\hline BLOOD \& BLOOD-FORMING ORGANS & $280-289$ & 0 & 0 & 0 & 2 \\
\hline MENTAL DISORDERS & $290-319$ & 2 & 0 & 0 & 9 \\
\hline -Non-Psychotic Disorders & $300-302,306-316$ & 2 & 0 & 0 & 9 \\
\hline NERVOUS SYSTEM (NS) \& SENSE ORGANS & $320-389$ & 1 & 2 & 0 & 41 \\
\hline -Hereditary/Degenerative Central NS Dis & 330-337 & 0 & 0 & 0 & 3 \\
\hline -Other Disorders of Central NS & $340-349$ & 0 & 0 & 0 & 4 \\
\hline -Disorders of Peripheral NS & $350-359$ & 0 & 0 & 0 & 8 \\
\hline -Disorders of Eye & $360-379$ & 0 & 2 & 0 & 12 \\
\hline -Diseases of Ear \& Mastoid & 380-389 & 1 & 0 & 0 & 14 \\
\hline CIRCULATORY SYSTEM & $390-459$ & 1 & 0 & 0 & 13 \\
\hline -Hypertensive Dis & $401-405$ & 1 & 0 & 0 & 3 \\
\hline -Ischemic Heart Dis & $410-414$ & 0 & 0 & 0 & 4 \\
\hline -Other Heart Dis & 420-429 & 0 & 0 & 0 & 2 \\
\hline -Cerebrovascular Dis & $430-438$ & 0 & 0 & 0 & 3 \\
\hline -Dis of Veins, Lymphatics, Other & 451-459 & 0 & 0 & 0 & 1 \\
\hline
\end{tabular}

(Continued)

*Only those diagnostic categories and gender/job category combinations with at least one occurrence appear in this table. 
Oak Ridge National Laboratory 2010

Absence Data

Appendix I. Number of Diagnoses in Each Diagnostic Category by Gender and Job Category*

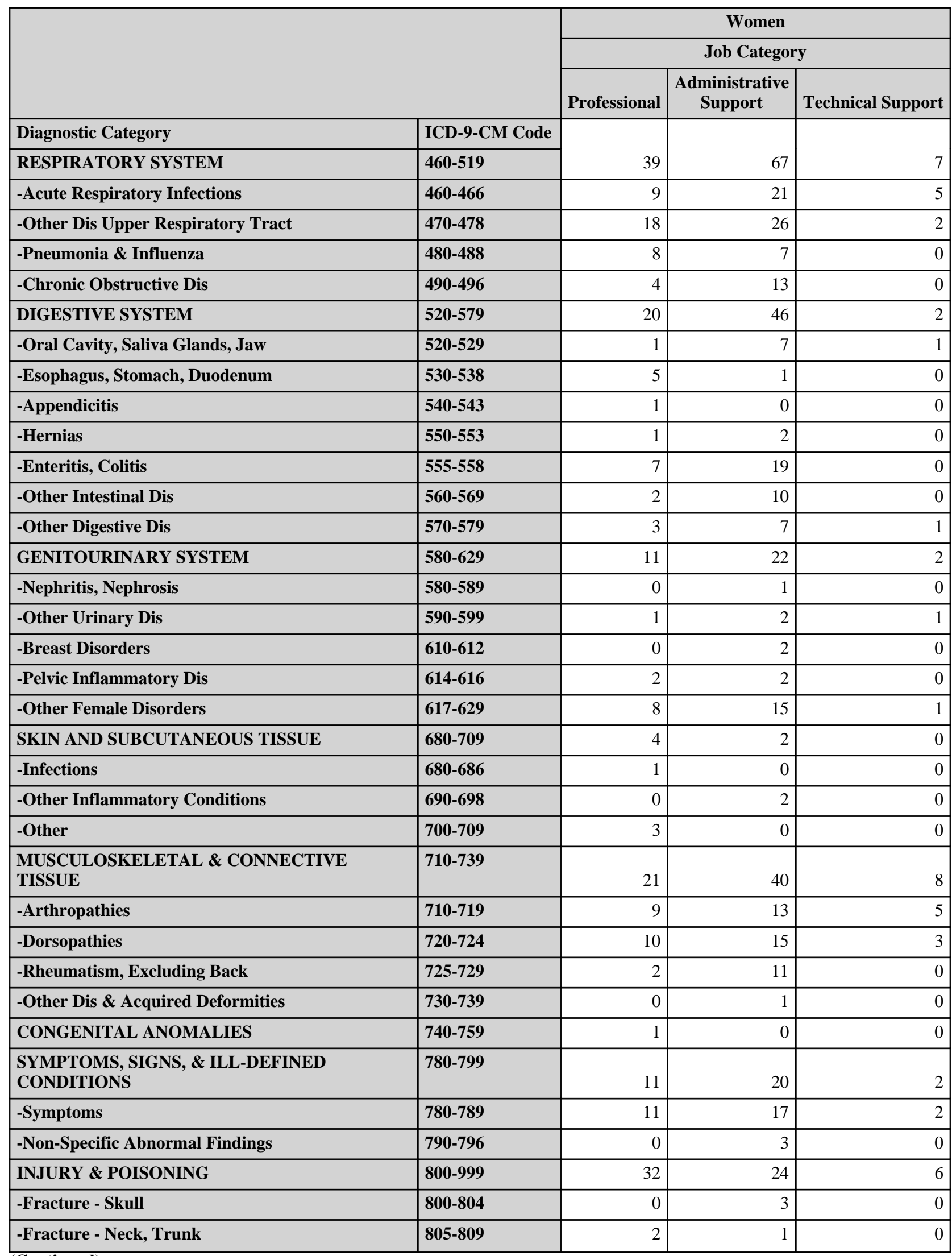

(Continued)

*Only those diagnostic categories and gender/job category combinations with at least one occurrence appear in this table. 
Oak Ridge National Laboratory 2010

Absence Data

Appendix I. Number of Diagnoses in Each Diagnostic Category by Gender and Job Category*

\begin{tabular}{|c|c|c|c|c|c|}
\hline & & \multicolumn{4}{|c|}{ Women } \\
\hline & & \multicolumn{3}{|c|}{ Job Category } & \multirow[b]{2}{*}{ TOTAL } \\
\hline & & Service & Security and Fire & Crafts & \\
\hline Diagnostic Category & ICD-9-CM Code & \multirow[b]{2}{*}{5} & \multirow[b]{2}{*}{2} & \multirow[b]{2}{*}{1} & \multirow[b]{2}{*}{121} \\
\hline RESPIRATORY SYSTEM & $460-519$ & & & & \\
\hline -Acute Respiratory Infections & $460-466$ & 1 & 0 & 1 & 37 \\
\hline -Other Dis Upper Respiratory Tract & $470-478$ & 0 & 0 & 0 & 46 \\
\hline -Pneumonia \& Influenza & $480-488$ & 1 & 1 & 0 & 17 \\
\hline -Chronic Obstructive Dis & $490-496$ & 3 & 1 & 0 & 21 \\
\hline DIGESTIVE SYSTEM & $520-579$ & 3 & 0 & 2 & 73 \\
\hline -Oral Cavity, Saliva Glands, Jaw & $520-529$ & 2 & 0 & 1 & 12 \\
\hline -Esophagus, Stomach, Duodenum & $530-538$ & 0 & 0 & 0 & 6 \\
\hline -Appendicitis & $540-543$ & 0 & 0 & 0 & 1 \\
\hline -Hernias & $550-553$ & 0 & 0 & 0 & 3 \\
\hline -Enteritis, Colitis & $555-558$ & 1 & 0 & 0 & 27 \\
\hline -Other Intestinal Dis & $560-569$ & 0 & 0 & 0 & 12 \\
\hline -Other Digestive Dis & $570-579$ & 0 & 0 & 1 & 12 \\
\hline GENITOURINARY SYSTEM & $580-629$ & 3 & 0 & 2 & 40 \\
\hline -Nephritis, Nephrosis & $580-589$ & 0 & 0 & 0 & 1 \\
\hline -Other Urinary Dis & $590-599$ & 1 & 0 & 0 & 5 \\
\hline -Breast Disorders & $610-612$ & 2 & 0 & 1 & 5 \\
\hline -Pelvic Inflammatory Dis & 614-616 & 0 & 0 & 0 & 4 \\
\hline -Other Female Disorders & $617-629$ & 0 & 0 & 1 & 25 \\
\hline SKIN AND SUBCUTANEOUS TISSUE & $680-709$ & 2 & 0 & 0 & 8 \\
\hline -Infections & $680-686$ & 1 & 0 & 0 & 2 \\
\hline -Other Inflammatory Conditions & $690-698$ & 0 & 0 & 0 & 2 \\
\hline -Other & 700-709 & 1 & 0 & 0 & 4 \\
\hline $\begin{array}{l}\text { MUSCULOSKELETAL \& CONNECTIVE } \\
\text { TISSUE }\end{array}$ & 710-739 & 9 & 0 & 8 & 86 \\
\hline -Arthropathies & $710-719$ & 6 & 0 & 1 & 34 \\
\hline -Dorsopathies & $720-724$ & 0 & 0 & 4 & 32 \\
\hline -Rheumatism, Excluding Back & 725-729 & 1 & 0 & 1 & 15 \\
\hline -Other Dis \& Acquired Deformities & $730-739$ & 2 & 0 & 2 & 5 \\
\hline CONGENITAL ANOMALIES & $740-759$ & 0 & 0 & 0 & 1 \\
\hline $\begin{array}{l}\text { SYMPTOMS, SIGNS, \& ILL-DEFINED } \\
\text { CONDITIONS }\end{array}$ & 780-799 & 0 & 0 & 0 & 33 \\
\hline -Symptoms & 780-789 & 0 & 0 & 0 & 30 \\
\hline -Non-Specific Abnormal Findings & $790-796$ & 0 & 0 & 0 & 3 \\
\hline INJURY \& POISONING & $800-999$ & 2 & 0 & 3 & 67 \\
\hline -Fracture - Skull & $800-804$ & 0 & 0 & 1 & 4 \\
\hline -Fracture - Neck, Trunk & \begin{tabular}{|l|}
$805-809$ \\
\end{tabular} & 0 & 0 & 0 & 3 \\
\hline
\end{tabular}

(Continued)

*Only those diagnostic categories and gender/job category combinations with at least one occurrence appear in this table. 
Oak Ridge National Laboratory 2010

Absence Data

Appendix I. Number of Diagnoses in Each Diagnostic Category by Gender and Job Category*

\begin{tabular}{|c|c|c|c|c|}
\hline & & \multicolumn{3}{|c|}{ Women } \\
\hline & & \multicolumn{3}{|c|}{ Job Category } \\
\hline & & Professional & $\begin{array}{l}\text { Administrative } \\
\text { Support }\end{array}$ & Technical Support \\
\hline Diagnostic Category & ICD-9-CM Code & \multirow[b]{2}{*}{0} & \multirow[b]{2}{*}{2} & \multirow[b]{2}{*}{1} \\
\hline -Fracture - Upper Limb & 810-819 & & & \\
\hline -Fracture - Lower Limb & $820-829$ & 6 & 2 & 1 \\
\hline -Dislocation & 830-839 & 6 & 5 & 1 \\
\hline -Sprains \& Strains - Back & 846-847 & 2 & 2 & 2 \\
\hline -Sprains \& Strains - Other & $840-845,848$ & 8 & 3 & 1 \\
\hline -Open Wound - Head, Neck, Trunk & 870-879 & 0 & 2 & 0 \\
\hline -Open Wound - Lower Limb & 890-897 & 1 & 0 & 0 \\
\hline -Late Effects of Accident & 905-909 & 0 & 0 & 0 \\
\hline -Contusion & $920-924$ & 0 & 3 & 0 \\
\hline -Unspecified Effects - External Causes & 990-995 & 1 & 0 & 0 \\
\hline -Complications of Surgical/Medical Care & 996-999 & 6 & 1 & 0 \\
\hline $\begin{array}{l}\text { HEALTH STATUS/HEALTH SERVICE } \\
\text { CONTACT }\end{array}$ & V01-V89 & 6 & 1 & 1 \\
\hline -Personal \& Family History & V10-V19 & 1 & 0 & 0 \\
\hline -Health Services Reproduction/Development & V20-V29 & 1 & 0 & 0 \\
\hline -Health Status & V40-V49 & 2 & 0 & 0 \\
\hline -Specific Procedures/Aftercare & V50-V59 & 2 & 1 & 1 \\
\hline
\end{tabular}

*Only those diagnostic categories and gender/job category combinations with at least one occurrence appear in this table. 
Oak Ridge National Laboratory 2010

Absence Data

Appendix I. Number of Diagnoses in Each Diagnostic Category by Gender and Job Category*

\begin{tabular}{|c|c|c|c|c|c|}
\hline & & \multicolumn{4}{|c|}{ Women } \\
\hline & & \multicolumn{3}{|c|}{ Job Category } & \multirow[b]{2}{*}{ TOTAL } \\
\hline & & Service & Security and Fire & Crafts & \\
\hline Diagnostic Category & ICD-9-CM Code & \multirow[b]{2}{*}{0} & \multirow[b]{2}{*}{0} & \multirow[b]{2}{*}{1} & \multirow[b]{2}{*}{4} \\
\hline -Fracture - Upper Limb & 810-819 & & & & \\
\hline -Fracture - Lower Limb & $820-829$ & 0 & 0 & 0 & 9 \\
\hline -Dislocation & $830-839$ & 0 & 0 & 0 & 12 \\
\hline -Sprains \& Strains - Back & 846-847 & 0 & 0 & 0 & 6 \\
\hline -Sprains \& Strains - Other & $840-845,848$ & 1 & 0 & 0 & 13 \\
\hline -Open Wound - Head, Neck, Trunk & 870-879 & 0 & 0 & 0 & 2 \\
\hline -Open Wound - Lower Limb & $890-897$ & 0 & 0 & 0 & 1 \\
\hline -Late Effects of Accident & $905-909$ & 1 & 0 & 0 & 1 \\
\hline -Contusion & $920-924$ & 0 & 0 & 1 & 4 \\
\hline -Unspecified Effects - External Causes & $990-995$ & 0 & 0 & 0 & 1 \\
\hline -Complications of Surgical/Medical Care & 996-999 & 0 & 0 & 0 & 7 \\
\hline $\begin{array}{l}\text { HEALTH STATUS/HEALTH SERVICE } \\
\text { CONTACT }\end{array}$ & V01-V89 & 2 & 0 & 0 & 10 \\
\hline -Personal \& Family History & V10-V19 & 0 & 0 & 0 & 1 \\
\hline -Health Services Reproduction/Development & V20-V29 & 0 & 0 & 0 & 1 \\
\hline -Health Status & V40-V49 & 0 & 0 & 0 & 2 \\
\hline -Specific Procedures/Aftercare & V50-V59 & 2 & 0 & 0 & 6 \\
\hline
\end{tabular}

\begin{tabular}{|c|c|c|c|c|c|c|c|}
\hline & \multicolumn{7}{|c|}{ Women } \\
\hline & \multicolumn{6}{|c|}{ Job Category } & \multirow[b]{2}{*}{ TOTAL } \\
\hline & Professional & $\begin{array}{c}\text { Administrative } \\
\text { Support }\end{array}$ & Technical Support & Service & Security and Fire & Crafts & \\
\hline Diagnostic Category & \multirow[b]{2}{*}{193} & \multirow[b]{2}{*}{272} & \multirow[b]{2}{*}{36} & \multirow[b]{2}{*}{32} & \multirow[b]{2}{*}{5} & \multirow[b]{2}{*}{16} & \multirow[b]{2}{*}{554} \\
\hline Total & & & & & & & \\
\hline
\end{tabular}

*Only those diagnostic categories and gender/job category combinations with at least one occurrence appear in this table. 
Oak Ridge National Laboratory 2010

Absence Data

Appendix I. Number of Diagnoses in Each Diagnostic Category by Gender and Job Category*

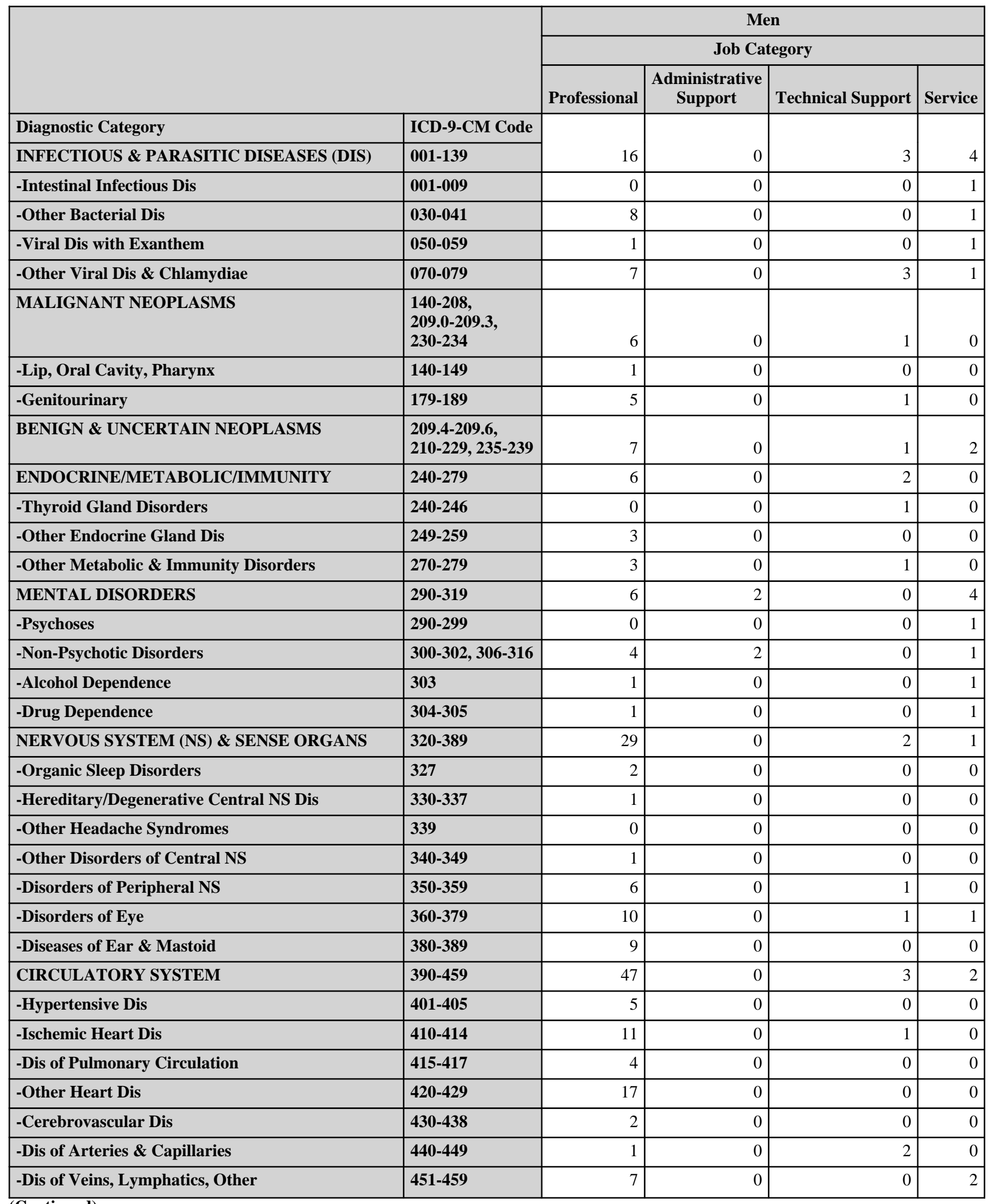

*Only those diagnostic categories and gender/job category combinations with at least one occurrence appear in this table. 
Oak Ridge National Laboratory 2010

Absence Data

Appendix I. Number of Diagnoses in Each Diagnostic Category by Gender and Job Category*

\begin{tabular}{|c|c|c|c|c|c|}
\hline & & \multicolumn{4}{|c|}{ Men } \\
\hline & & \multicolumn{3}{|c|}{ Job Category } & \multirow[b]{2}{*}{ TOTAL } \\
\hline & & Security and Fire & Crafts & Line Operators & \\
\hline Diagnostic Category & ICD-9-CM Code & \multirow[b]{2}{*}{0} & \multirow[b]{2}{*}{3} & \multirow[b]{2}{*}{0} & \multirow[b]{2}{*}{26} \\
\hline INFECTIOUS \& PARASITIC DISEASES (DIS) & 001-139 & & & & \\
\hline -Intestinal Infectious Dis & 001-009 & 0 & 0 & 0 & 1 \\
\hline -Other Bacterial Dis & 030-041 & 0 & 2 & 0 & 11 \\
\hline -Viral Dis with Exanthem & 050-059 & 0 & 1 & 0 & 3 \\
\hline -Other Viral Dis \& Chlamydiae & 070-079 & 0 & 0 & 0 & 11 \\
\hline MALIGNANT NEOPLASMS & \begin{tabular}{|l|}
$140-208$ \\
$209.0-209.3$ \\
$230-234$
\end{tabular} & 0 & 0 & 0 & 7 \\
\hline -Lip, Oral Cavity, Pharynx & $140-149$ & 0 & 0 & 0 & 1 \\
\hline -Genitourinary & 179-189 & 0 & 0 & 0 & 6 \\
\hline BENIGN \& UNCERTAIN NEOPLASMS & $\begin{array}{l}209.4-209.6 \\
210-229,235-239\end{array}$ & 1 & 5 & 0 & 16 \\
\hline ENDOCRINE/METABOLIC/IMMUNITY & $240-279$ & 0 & 2 & 0 & 10 \\
\hline -Thyroid Gland Disorders & $240-246$ & 0 & 0 & 0 & 1 \\
\hline -Other Endocrine Gland Dis & $249-259$ & 0 & 0 & 0 & 3 \\
\hline -Other Metabolic \& Immunity Disorders & 270-279 & 0 & 2 & 0 & 6 \\
\hline MENTAL DISORDERS & 290-319 & 0 & 1 & 1 & 14 \\
\hline -Psychoses & 290-299 & 0 & 0 & 0 & 1 \\
\hline -Non-Psychotic Disorders & $300-302,306-316$ & 0 & 0 & 0 & 7 \\
\hline -Alcohol Dependence & 303 & 0 & 0 & 0 & 2 \\
\hline -Drug Dependence & $304-305$ & 0 & 1 & 1 & 4 \\
\hline NERVOUS SYSTEM (NS) \& SENSE ORGANS & 320-389 & 0 & 12 & 5 & 49 \\
\hline -Organic Sleep Disorders & 327 & 0 & 1 & 0 & 3 \\
\hline -Hereditary/Degenerative Central NS Dis & 330-337 & 0 & 0 & 0 & 1 \\
\hline -Other Headache Syndromes & 339 & 0 & 1 & 0 & 1 \\
\hline -Other Disorders of Central NS & 340-349 & 0 & 0 & 1 & 2 \\
\hline -Disorders of Peripheral NS & $350-359$ & 0 & 5 & 2 & 14 \\
\hline -Disorders of Eye & $360-379$ & 0 & 1 & 1 & 14 \\
\hline -Diseases of Ear \& Mastoid & 380-389 & 0 & 4 & 1 & $\overline{14}$ \\
\hline CIRCULATORY SYSTEM & $390-459$ & 1 & 8 & 1 & 62 \\
\hline -Hypertensive Dis & $401-405$ & 0 & 2 & 0 & 7 \\
\hline -Ischemic Heart Dis & $410-414$ & 0 & 3 & 1 & 16 \\
\hline -Dis of Pulmonary Circulation & $415-417$ & 0 & 0 & 0 & 4 \\
\hline -Other Heart Dis & $420-429$ & 0 & 2 & 0 & 19 \\
\hline -Cerebrovascular Dis & $430-438$ & 0 & 1 & 0 & 3 \\
\hline -Dis of Arteries \& Capillaries & $440-449$ & 0 & 0 & 0 & 3 \\
\hline -Dis of Veins, Lymphatics, Other & $451-459$ & 1 & 0 & 0 & 10 \\
\hline
\end{tabular}

*Only those diagnostic categories and gender/job category combinations with at least one occurrence appear in this table. 
Oak Ridge National Laboratory 2010

Absence Data

Appendix I. Number of Diagnoses in Each Diagnostic Category by Gender and Job Category*

\begin{tabular}{|c|c|c|c|c|c|}
\hline & \multicolumn{4}{|c|}{ Men } \\
\hline & & \multicolumn{4}{|c|}{ Job Category } \\
\hline & & Professional & $\begin{array}{c}\text { Administrative } \\
\text { Support }\end{array}$ & Technical Support & Service \\
\hline Diagnostic Category & ICD-9-CM Code & \multirow[b]{2}{*}{39} & \multirow[b]{2}{*}{0} & \multirow[b]{2}{*}{12} & \multirow[b]{2}{*}{9} \\
\hline RESPIRATORY SYSTEM & $460-519$ & & & & \\
\hline -Acute Respiratory Infections & $460-466$ & 8 & 0 & 4 & 3 \\
\hline -Other Dis Upper Respiratory Tract & $470-478$ & 19 & 0 & 5 & 0 \\
\hline -Pneumonia \& Influenza & $480-488$ & 5 & 0 & 1 & 3 \\
\hline -Chronic Obstructive Dis & $490-496$ & 6 & 0 & 2 & 3 \\
\hline -Other Respiratory Dis & $510-519$ & 1 & 0 & 0 & 0 \\
\hline DIGESTIVE SYSTEM & $520-579$ & 53 & 1 & 21 & 9 \\
\hline -Oral Cavity, Saliva Glands, Jaw & $520-529$ & 5 & 0 & 1 & 0 \\
\hline -Esophagus, Stomach, Duodenum & $530-538$ & 2 & 0 & 0 & 2 \\
\hline -Appendicitis & $540-543$ & 2 & 1 & 2 & 0 \\
\hline -Hernias & $550-553$ & 20 & 0 & 8 & 1 \\
\hline -Enteritis, Colitis & $555-558$ & 8 & 0 & 4 & 5 \\
\hline -Other Intestinal Dis & $560-569$ & 7 & 0 & 2 & 0 \\
\hline -Other Digestive Dis & $570-579$ & 9 & 0 & 4 & 1 \\
\hline GENITOURINARY SYSTEM & $580-629$ & 13 & 0 & 2 & 0 \\
\hline -Nephritis, Nephrosis & $580-589$ & 2 & 0 & 0 & 0 \\
\hline -Other Urinary Dis & $590-599$ & 7 & 0 & 0 & 0 \\
\hline -Male Genital Organ Dis & $600-608$ & 4 & 0 & 2 & 0 \\
\hline SKIN AND SUBCUTANEOUS TISSUE & 680-709 & 5 & 0 & 0 & 0 \\
\hline -Infections & $680-686$ & 2 & 0 & 0 & 0 \\
\hline -Other Inflammatory Conditions & 690-698 & 0 & 0 & 0 & 0 \\
\hline -Other & 700-709 & 3 & 0 & 0 & 0 \\
\hline $\begin{array}{l}\text { MUSCULOSKELETAL \& CONNECTIVE } \\
\text { TISSUE }\end{array}$ & 710-739 & 70 & 0 & 13 & 6 \\
\hline -Arthropathies & $710-719$ & 27 & 0 & 7 & 2 \\
\hline -Dorsopathies & $720-724$ & 19 & 0 & 3 & 1 \\
\hline -Rheumatism, Excluding Back & $725-729$ & 20 & 0 & 1 & 3 \\
\hline -Other Dis \& Acquired Deformities & 730-739 & 4 & 0 & 2 & 0 \\
\hline CONGENITAL ANOMALIES & $740-759$ & 1 & 0 & 0 & 0 \\
\hline $\begin{array}{l}\text { SYMPTOMS, SIGNS, \& ILL-DEFINED } \\
\text { CONDITIONS }\end{array}$ & 780-799 & 20 & 0 & 13 & 4 \\
\hline -Symptoms & 780-789 & 18 & 0 & 13 & 4 \\
\hline -Non-Specific Abnormal Findings & $790-796$ & 2 & 0 & 0 & 0 \\
\hline INJURY \& POISONING & $800-999$ & 96 & 1 & 15 & 5 \\
\hline -Fracture - Skull & $800-804$ & 1 & 0 & 1 & 0 \\
\hline -Fracture - Neck, Trunk & 805-809 & 14 & 0 & 1 & 0 \\
\hline -Fracture - Upper Limb & 810-819 & 5 & 0 & 2 & 0 \\
\hline
\end{tabular}

(Continued)

*Only those diagnostic categories and gender/job category combinations with at least one occurrence appear in this table. 
Oak Ridge National Laboratory 2010

Absence Data

Appendix I. Number of Diagnoses in Each Diagnostic Category by Gender and Job Category*

\begin{tabular}{|c|c|c|c|c|c|}
\hline & & \multicolumn{4}{|c|}{ Men } \\
\hline & & \multicolumn{3}{|c|}{ Job Category } & \multirow[b]{2}{*}{ TOTAL } \\
\hline & & Security and Fire & Crafts & Line Operators & \\
\hline Diagnostic Category & ICD-9-CM Code & \multirow[b]{2}{*}{4} & \multirow[b]{2}{*}{24} & \multirow[b]{2}{*}{4} & \multirow[b]{2}{*}{92} \\
\hline RESPIRATORY SYSTEM & 460-519 & & & & \\
\hline -Acute Respiratory Infections & $460-466$ & 0 & 6 & 0 & 21 \\
\hline -Other Dis Upper Respiratory Tract & $470-478$ & 1 & 10 & 1 & 36 \\
\hline -Pneumonia \& Influenza & $480-488$ & 0 & 4 & 1 & 14 \\
\hline -Chronic Obstructive Dis & $490-496$ & 3 & 4 & 2 & 20 \\
\hline -Other Respiratory Dis & $510-519$ & 0 & 0 & 0 & 1 \\
\hline DIGESTIVE SYSTEM & $520-579$ & 5 & 23 & 2 & 114 \\
\hline -Oral Cavity, Saliva Glands, Jaw & $520-529$ & 1 & 0 & 0 & 7 \\
\hline -Esophagus, Stomach, Duodenum & $530-538$ & 0 & 4 & 0 & 8 \\
\hline -Appendicitis & $540-543$ & 1 & 0 & 0 & 6 \\
\hline -Hernias & $550-553$ & 1 & 10 & 1 & 41 \\
\hline -Enteritis, Colitis & $555-558$ & 1 & 4 & 0 & 22 \\
\hline -Other Intestinal Dis & $560-569$ & 0 & 3 & 0 & 12 \\
\hline -Other Digestive Dis & $570-579$ & 1 & 2 & 1 & 18 \\
\hline GENITOURINARY SYSTEM & $580-629$ & 2 & 13 & 2 & 32 \\
\hline -Nephritis, Nephrosis & $580-589$ & 0 & 0 & 0 & 2 \\
\hline -Other Urinary Dis & $590-599$ & 1 & 10 & 2 & 20 \\
\hline -Male Genital Organ Dis & $600-608$ & 1 & 3 & 0 & 10 \\
\hline SKIN AND SUBCUTANEOUS TISSUE & 680-709 & 1 & 8 & 0 & 14 \\
\hline -Infections & $680-686$ & 0 & 2 & 0 & 4 \\
\hline -Other Inflammatory Conditions & 690-698 & 0 & 2 & 0 & 2 \\
\hline -Other & 700-709 & 1 & 4 & 0 & 8 \\
\hline $\begin{array}{l}\text { MUSCULOSKELETAL \& CONNECTIVE } \\
\text { TISSUE }\end{array}$ & $710-739$ & 4 & 31 & 6 & 130 \\
\hline -Arthropathies & 710-719 & 0 & 11 & 1 & 48 \\
\hline -Dorsopathies & $720-724$ & 4 & 12 & 4 & 43 \\
\hline -Rheumatism, Excluding Back & \begin{tabular}{|l|}
$725-729$ \\
\end{tabular} & 0 & 6 & 0 & 30 \\
\hline -Other Dis \& Acquired Deformities & 730-739 & 0 & 2 & 1 & 9 \\
\hline CONGENITAL ANOMALIES & $740-759$ & 0 & 0 & 0 & 1 \\
\hline $\begin{array}{l}\text { SYMPTOMS, SIGNS, \& ILL-DEFINED } \\
\text { CONDITIONS }\end{array}$ & $780-799$ & 1 & 12 & 1 & 51 \\
\hline -Symptoms & 780-789 & 1 & 9 & 1 & 46 \\
\hline -Non-Specific Abnormal Findings & $790-796$ & 0 & 3 & 0 & 5 \\
\hline INJURY \& POISONING & $800-999$ & 4 & 37 & 6 & 164 \\
\hline -Fracture - Skull & $800-804$ & 0 & 1 & 0 & 3 \\
\hline -Fracture - Neck, Trunk & $805-809$ & 0 & 1 & 1 & 17 \\
\hline -Fracture - Upper Limb & $810-819$ & 0 & 2 & 0 & 9 \\
\hline
\end{tabular}

(Continued)

*Only those diagnostic categories and gender/job category combinations with at least one occurrence appear in this table. 
Oak Ridge National Laboratory 2010

Absence Data

Appendix I. Number of Diagnoses in Each Diagnostic Category by Gender and Job Category*

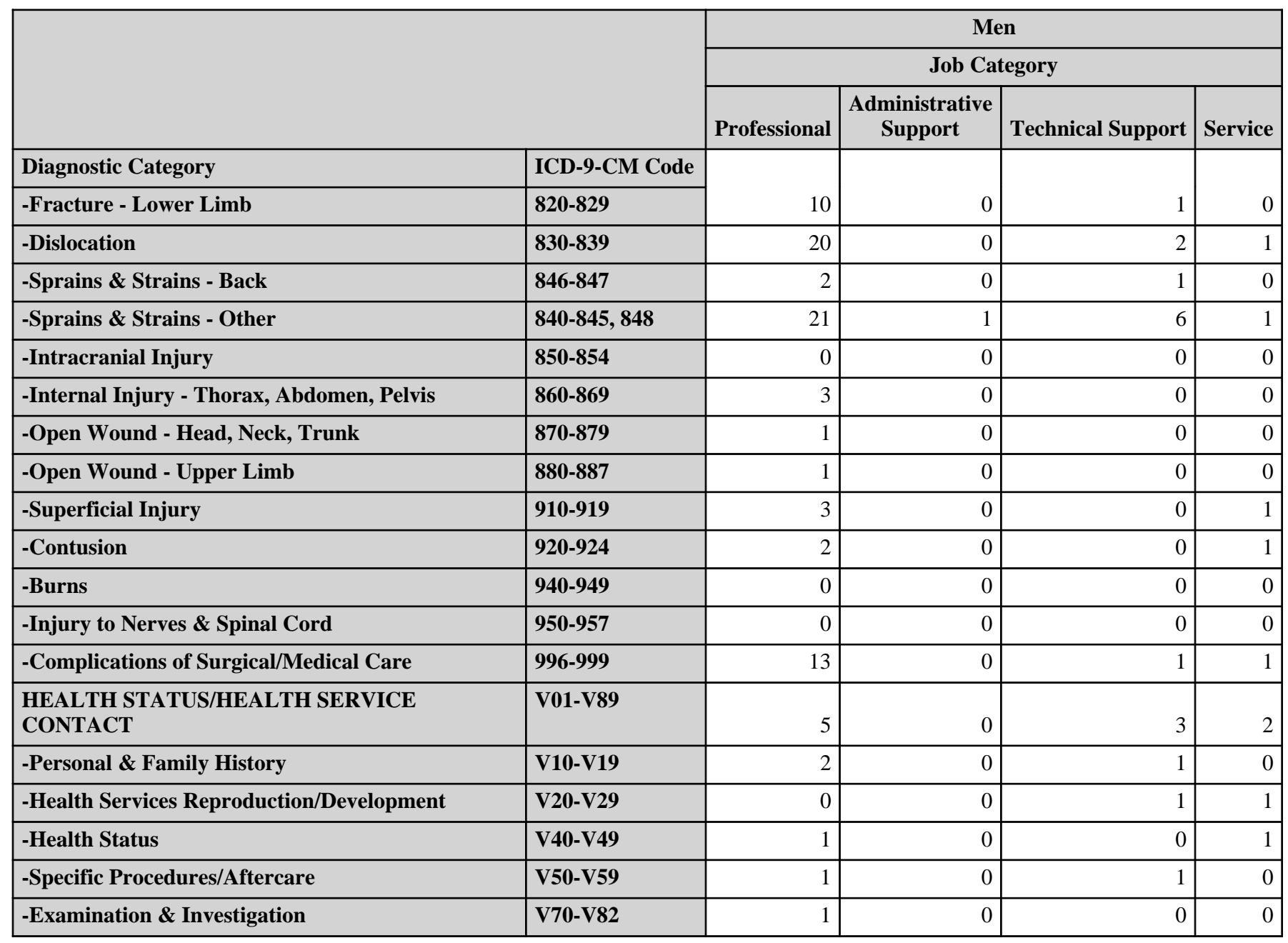

*Only those diagnostic categories and gender/job category combinations with at least one occurrence appear in this table. 
Oak Ridge National Laboratory 2010

Absence Data

Appendix I. Number of Diagnoses in Each Diagnostic Category by Gender and Job Category*

\begin{tabular}{|c|c|c|c|c|c|}
\hline & & \multicolumn{4}{|c|}{ Men } \\
\hline & & \multicolumn{3}{|c|}{ Job Category } & \multirow[b]{2}{*}{ TOTAL } \\
\hline & & Security and Fire & Crafts & Line Operators & \\
\hline Diagnostic Category & ICD-9-CM Code & \multirow[b]{2}{*}{1} & \multirow[b]{2}{*}{1} & \multirow[b]{2}{*}{1} & \multirow[b]{2}{*}{14} \\
\hline -Fracture - Lower Limb & $820-829$ & & & & \\
\hline -Dislocation & $830-839$ & 0 & 4 & 0 & 27 \\
\hline -Sprains \& Strains - Back & 846-847 & 2 & 3 & 1 & 9 \\
\hline -Sprains \& Strains - Other & $840-845,848$ & 1 & 10 & 2 & 42 \\
\hline -Intracranial Injury & $850-854$ & 0 & 1 & 1 & 2 \\
\hline -Internal Injury - Thorax, Abdomen, Pelvis & $860-869$ & 0 & 0 & 0 & 3 \\
\hline -Open Wound - Head, Neck, Trunk & $870-879$ & 0 & 1 & 0 & 2 \\
\hline -Open Wound - Upper Limb & 880-887 & 0 & 1 & 0 & 2 \\
\hline -Superficial Injury & 910-919 & 0 & 2 & 0 & 6 \\
\hline -Contusion & $920-924$ & 0 & 5 & 0 & 8 \\
\hline -Burns & 940-949 & 0 & 4 & 0 & 4 \\
\hline -Injury to Nerves \& Spinal Cord & 950-957 & 0 & 1 & 0 & 1 \\
\hline -Complications of Surgical/Medical Care & 996-999 & 0 & 0 & 0 & 15 \\
\hline $\begin{array}{l}\text { HEALTH STATUS/HEALTH SERVICE } \\
\text { CONTACT }\end{array}$ & V01-V89 & 0 & 1 & 0 & 11 \\
\hline -Personal \& Family History & V10-V19 & 0 & 1 & 0 & 4 \\
\hline -Health Services Reproduction/Development & V20-V29 & 0 & 0 & 0 & 2 \\
\hline -Health Status & V40-V49 & 0 & 0 & 0 & 2 \\
\hline -Specific Procedures/Aftercare & V50-V59 & 0 & 0 & 0 & 2 \\
\hline -Examination \& Investigation & V70-V82 & 0 & 0 & 0 & 1 \\
\hline
\end{tabular}

\begin{tabular}{|c|c|c|c|c|c|c|c|c|}
\hline & \multicolumn{8}{|c|}{ Men } \\
\hline & \multicolumn{7}{|c|}{ Job Category } & \multirow[b]{2}{*}{ TOTAL } \\
\hline & Professional & $\begin{array}{c}\text { Administrative } \\
\text { Support }\end{array}$ & $\begin{array}{l}\text { Technical } \\
\text { Support }\end{array}$ & Service & $\begin{array}{l}\text { Security and } \\
\text { Fire }\end{array}$ & Crafts & $\begin{array}{c}\text { Line } \\
\text { Operators }\end{array}$ & \\
\hline Diagnostic Category & \multirow[b]{2}{*}{419} & \multirow[b]{2}{*}{4} & \multirow[b]{2}{*}{91} & \multirow[b]{2}{*}{48} & \multirow[b]{2}{*}{23} & \multirow[b]{2}{*}{180} & \multirow[b]{2}{*}{28} & \multirow[b]{2}{*}{793} \\
\hline Total & & & & & & & & \\
\hline
\end{tabular}

*Only those diagnostic categories and gender/job category combinations with at least one occurrence appear in this table. 
Oak Ridge National Laboratory 2010

Absence Data

Appendix J. Total Number of Calendar Days Absent in Each Diagnostic Category by Gender and Job Category*

\begin{tabular}{|c|c|c|c|c|}
\hline & & \multicolumn{3}{|c|}{ Women } \\
\hline & & \multicolumn{3}{|c|}{ Job Category } \\
\hline & & Professional & $\begin{array}{l}\text { Administrative } \\
\text { Support }\end{array}$ & Technical Support \\
\hline Diagnostic Category & ICD-9-CM Code & \multirow[b]{2}{*}{145} & \multirow[b]{2}{*}{56} & \multirow[b]{2}{*}{14} \\
\hline INFECTIOUS \& PARASITIC DISEASES (DIS) & 001-139 & & & \\
\hline MALIGNANT NEOPLASMS & $\begin{array}{l}140-208 \\
209.0-209.3 \\
230-234\end{array}$ & 176 & 22 & 47 \\
\hline BENIGN \& UNCERTAIN NEOPLASMS & $\begin{array}{l}209.4-209.6 \\
210-229,235-239\end{array}$ & 35 & 208 & 0 \\
\hline ENDOCRINE/METABOLIC/IMMUNITY & $240-279$ & 56 & 20 & 0 \\
\hline BLOOD \& BLOOD-FORMING ORGANS & $280-289$ & 0 & 19 & 0 \\
\hline MENTAL DISORDERS & 290-319 & 110 & 28 & 280 \\
\hline NERVOUS SYSTEM (NS) \& SENSE ORGANS & $320-389$ & 381 & 281 & 56 \\
\hline CIRCULATORY SYSTEM & $390-459$ & 130 & 184 & 0 \\
\hline RESPIRATORY SYSTEM & $460-519$ & 315 & 504 & 23 \\
\hline DIGESTIVE SYSTEM & $520-579$ & 300 & 556 & 24 \\
\hline GENITOURINARY SYSTEM & $580-629$ & 250 & 507 & 35 \\
\hline SKIN AND SUBCUTANEOUS TISSUE & $680-709$ & 71 & 8 & 0 \\
\hline $\begin{array}{l}\text { MUSCULOSKELETAL \& CONNECTIVE } \\
\text { TISSUE }\end{array}$ & $710-739$ & 736 & 1,142 & 153 \\
\hline CONGENITAL ANOMALIES & $740-759$ & 22 & 0 & 0 \\
\hline $\begin{array}{l}\text { SYMPTOMS, SIGNS, \& ILL-DEFINED } \\
\text { CONDITIONS }\end{array}$ & 780-799 & 75 & 241 & 29 \\
\hline INJURY \& POISONING & $800-999$ & 711 & 586 & 40 \\
\hline
\end{tabular}

\footnotetext{
*Absences with >1 ICD-9-CM code in the same diagnostic category were counted only once. Only those diagnostic categories and gender/job category combinations with at least one occurrence appear in this table.
} 
Oak Ridge National Laboratory 2010

Absence Data

Appendix J. Total Number of Calendar Days Absent in Each Diagnostic Category by Gender and Job Category*

\begin{tabular}{|c|c|c|c|c|c|}
\hline & & \multicolumn{4}{|c|}{ Women } \\
\hline & & \multicolumn{3}{|c|}{ Job Category } & \multirow[b]{2}{*}{ TOTAL } \\
\hline & & Service & Security and Fire & Crafts & \\
\hline Diagnostic Category & ICD-9-CM Code & \multirow[b]{2}{*}{21} & \multirow[b]{2}{*}{0} & \multirow[b]{2}{*}{0} & \multirow[b]{2}{*}{236} \\
\hline INFECTIOUS \& PARASITIC DISEASES (DIS) & 001-139 & & & & \\
\hline MALIGNANT NEOPLASMS & $\begin{array}{l}140-208, \\
209.0-209.3 \\
230-234\end{array}$ & 0 & 0 & 0 & 245 \\
\hline BENIGN \& UNCERTAIN NEOPLASMS & \begin{tabular}{|l|}
$209.4-209.6$ \\
$210-229$, \\
\end{tabular} & 0 & 0 & 0 & 243 \\
\hline ENDOCRINE/METABOLIC/IMMUNITY & $240-279$ & 0 & 25 & 0 & 101 \\
\hline BLOOD \& BLOOD-FORMING ORGANS & 280-289 & 0 & 0 & 0 & 19 \\
\hline MENTAL DISORDERS & 290-319 & 111 & 0 & 0 & 529 \\
\hline NERVOUS SYSTEM (NS) \& SENSE ORGANS & $320-389$ & 10 & 30 & 0 & 758 \\
\hline CIRCULATORY SYSTEM & $390-459$ & 10 & 0 & 0 & 324 \\
\hline RESPIRATORY SYSTEM & $460-519$ & 77 & 14 & 6 & 939 \\
\hline DIGESTIVE SYSTEM & $520-579$ & 14 & 0 & 51 & 945 \\
\hline GENITOURINARY SYSTEM & $580-629$ & 39 & 0 & 63 & 894 \\
\hline SKIN AND SUBCUTANEOUS TISSUE & 680-709 & 136 & 0 & 0 & 215 \\
\hline $\begin{array}{l}\text { MUSCULOSKELETAL \& CONNECTIVE } \\
\text { TISSUE }\end{array}$ & 710-739 & 518 & 0 & 482 & 3,031 \\
\hline CONGENITAL ANOMALIES & \begin{tabular}{|l|}
$740-759$ \\
\end{tabular} & 0 & 0 & 0 & 22 \\
\hline $\begin{array}{l}\text { SYMPTOMS, SIGNS, \& ILL-DEFINED } \\
\text { CONDITIONS }\end{array}$ & $780-799$ & 0 & 0 & 0 & 345 \\
\hline INJURY \& POISONING & 800-999 & 209 & 0 & 78 & 1,624 \\
\hline
\end{tabular}

\footnotetext{
*Absences with >1 ICD-9-CM code in the same diagnostic category were counted only once. Only those diagnostic categories and gender/job category combinations with at least one occurrence appear in this table.
} 
Oak Ridge National Laboratory 2010

Absence Data

Appendix J. Total Number of Calendar Days Absent in Each Diagnostic Category by Gender and Job Category*

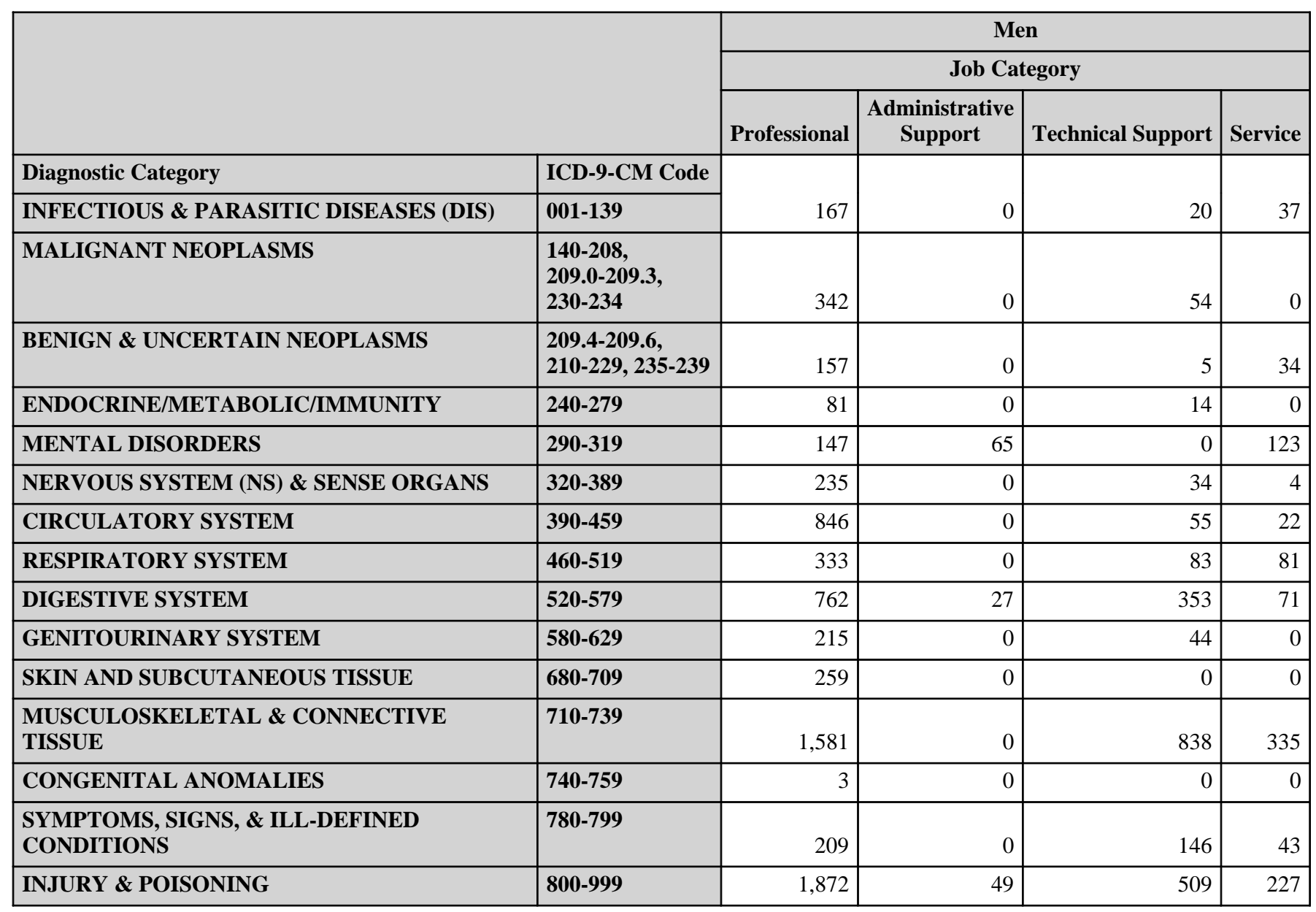

\footnotetext{
*Absences with >1 ICD-9-CM code in the same diagnostic category were counted only once. Only those diagnostic categories and gender/job category combinations with at least one occurrence appear in this table.
} 
Oak Ridge National Laboratory 2010

Absence Data

Appendix J. Total Number of Calendar Days Absent in Each Diagnostic Category by Gender and Job Category*

\begin{tabular}{|c|c|c|c|c|c|}
\hline & & \multicolumn{4}{|c|}{ Men } \\
\hline & & \multicolumn{3}{|c|}{ Job Category } & \multirow[b]{2}{*}{ TOTAL } \\
\hline & & Security and Fire & Crafts & Line Operators & \\
\hline Diagnostic Category & ICD-9-CM Code & \multirow[b]{2}{*}{0} & \multirow[b]{2}{*}{24} & \multirow[b]{2}{*}{0} & \multirow[b]{2}{*}{248} \\
\hline INFECTIOUS \& PARASITIC DISEASES (DIS) & 001-139 & & & & \\
\hline MALIGNANT NEOPLASMS & \begin{tabular}{|l|}
$140-208$ \\
$209.0-209.3$ \\
$230-234$
\end{tabular} & 0 & 0 & 0 & 396 \\
\hline BENIGN \& UNCERTAIN NEOPLASMS & \begin{tabular}{|l|}
$209.4-209.6$ \\
$210-229$, \\
\end{tabular} & 12 & 47 & 0 & 255 \\
\hline ENDOCRINE/METABOLIC/IMMUNITY & 240-279 & 0 & 69 & 0 & 164 \\
\hline MENTAL DISORDERS & $290-319$ & 0 & 8 & 26 & 369 \\
\hline NERVOUS SYSTEM (NS) \& SENSE ORGANS & 320-389 & 0 & 512 & 172 & 957 \\
\hline CIRCULATORY SYSTEM & $390-459$ & 42 & 229 & 17 & 1,211 \\
\hline RESPIRATORY SYSTEM & $460-519$ & 40 & 220 & 114 & 871 \\
\hline DIGESTIVE SYSTEM & $520-579$ & 117 & 484 & 70 & 1,884 \\
\hline GENITOURINARY SYSTEM & $580-629$ & 36 & 131 & 29 & 455 \\
\hline SKIN AND SUBCUTANEOUS TISSUE & $680-709$ & 66 & 368 & 0 & 693 \\
\hline $\begin{array}{l}\text { MUSCULOSKELETAL \& CONNECTIVE } \\
\text { TISSUE }\end{array}$ & $710-739$ & 69 & 1,777 & 593 & 5,193 \\
\hline CONGENITAL ANOMALIES & 740-759 & 0 & 0 & 0 & 3 \\
\hline $\begin{array}{l}\text { SYMPTOMS, SIGNS, \& ILL-DEFINED } \\
\text { CONDITIONS }\end{array}$ & \begin{tabular}{|l|l}
$\mid 780-799$ \\
\end{tabular} & 12 & 120 & 17 & 547 \\
\hline INJURY \& POISONING & $800-999$ & 54 & 1,264 & 316 & 4,291 \\
\hline
\end{tabular}

\footnotetext{
*Absences with >1 ICD-9-CM code in the same diagnostic category were counted only once. Only those diagnostic categories and gender/job category combinations with at least one occurrence appear in this table.
} 
Oak Ridge National Laboratory 2010

Absence Data

Appendix K. Age-Adjusted Illness and Injury Rates by Diagnostic Category*

Part 1. Men

\begin{tabular}{|c|c|c|c|c|c|}
\hline & & $\begin{array}{l}\text { Number of } \\
\text { Diagnoses }\end{array}$ & $\begin{array}{c}\text { Age-Adjusted } \\
\text { Rate per } 1,000 * *\end{array}$ & $\begin{array}{c}\text { Lower } 95 \% \\
\text { Confidence } \\
\text { Limit per } 1,000\end{array}$ & $\begin{array}{c}\text { Upper } 95 \% \\
\text { Confidence } \\
\text { Limit per } 1,000\end{array}$ \\
\hline Diagnostic Category & ICD-9-CM Code & & & & \\
\hline INFECTIOUS \& PARASITIC DISEASES (DIS) & 001-139 & 26 & 9.1 & 5.3 & 15.5 \\
\hline -Intestinal Infectious Dis & 001-009 & 1 & 1.1 & 0.2 & 8.0 \\
\hline -Other Bacterial Dis & 030-041 & 11 & 3.4 & 1.6 & 7.5 \\
\hline -Viral Dis with Exanthem & 050-059 & 3 & 0.6 & 0.2 & 1.9 \\
\hline -Other Viral Dis \& Chlamydiae & 070-079 & 11 & 3.9 & 1.7 & 9.2 \\
\hline MALIGNANT NEOPLASMS & $\begin{array}{l}140-208 \\
209.0-209.3 \\
230-234\end{array}$ & 7 & 1.3 & 0.6 & 2.8 \\
\hline -Lip, Oral Cavity, Pharynx & $140-149$ & 1 & 0.2 & 0.0 & 1.5 \\
\hline -Genitourinary & 179-189 & 6 & 1.1 & 0.5 & 2.5 \\
\hline BENIGN \& UNCERTAIN NEOPLASMS & \begin{tabular}{|l|}
$209.4-209.6$ \\
$210-229$, \\
$235-239$
\end{tabular} & 16 & 2.9 & 1.7 & 5.0 \\
\hline ENDOCRINE/METABOLIC/IMMUNITY & $240-279$ & 10 & 1.8 & 0.9 & 3.4 \\
\hline -Thyroid Gland Disorders & $240-246$ & 1 & 0.3 & 0.0 & 1.8 \\
\hline -Other Endocrine Gland Dis & $249-259$ & 3 & 0.4 & 0.1 & 1.3 \\
\hline -Other Metabolic \& Immunity Disorders & $270-279$ & 6 & 1.1 & 0.5 & 2.7 \\
\hline MENTAL DISORDERS & $290-319$ & 14 & 3.2 & 1.8 & 5.5 \\
\hline -Psychoses & 290-299 & 1 & 0.3 & 0.0 & 1.8 \\
\hline -Non-Psychotic Disorders & $300-302,306-316$ & 7 & 1.2 & 0.5 & 2.6 \\
\hline -Alcohol Dependence & 303 & 2 & 0.5 & 0.1 & 2.0 \\
\hline -Drug Dependence & $304-305$ & 4 & 1.2 & 0.5 & 3.3 \\
\hline NERVOUS SYSTEM (NS) \& SENSE ORGANS & 320-389 & 49 & 9.5 & 7.0 & 12.7 \\
\hline -Organic Sleep Disorders & 327 & 3 & 1.0 & 0.3 & 3.1 \\
\hline -Hereditary/Degenerative Central NS Dis & 330-337 & 1 & 0.1 & 0.0 & 1.0 \\
\hline -Other Headache Syndromes & 339 & 1 & 0.1 & 0.0 & 1.0 \\
\hline -Other Disorders of Central NS & $340-349$ & 2 & 0.5 & 0.1 & 2.0 \\
\hline -Disorders of Peripheral NS & $350-359$ & 14 & 2.6 & 1.5 & 4.5 \\
\hline -Disorders of Eye & $360-379$ & 14 & 2.5 & 1.5 & 4.4 \\
\hline -Diseases of Ear \& Mastoid & $380-389$ & 14 & 2.6 & 1.5 & 4.5 \\
\hline CIRCULATORY SYSTEM & $390-459$ & 62 & 11.3 & 8.7 & 14.8 \\
\hline -Hypertensive Dis & $401-405$ & 7 & 1.2 & 0.6 & 2.7 \\
\hline -Ischemic Heart Dis & $410-414$ & 16 & 2.6 & 1.6 & 4.3 \\
\hline -Dis of Pulmonary Circulation & $415-417$ & 4 & 0.6 & 0.2 & 1.7 \\
\hline -Other Heart Dis & $420-429$ & 19 & 3.4 & 2.1 & 5.5 \\
\hline -Cerebrovascular Dis & $430-438$ & 3 & 0.6 & 0.2 & 1.9 \\
\hline -Dis of Arteries \& Capillaries & $440-449$ & 3 & 0.8 & 0.2 & 2.5 \\
\hline
\end{tabular}

(Continued)

* Only those diagnostic categories with at least one occurrence appear in this table.

**Standardized to age distribution of 2000 U.S. population. 
Oak Ridge National Laboratory 2010

Absence Data

Appendix K. Age-Adjusted Illness and Injury Rates by Diagnostic Category*

Part 1. Men

\begin{tabular}{|c|c|c|c|c|c|}
\hline & & $\begin{array}{l}\text { Number of } \\
\text { Diagnoses }\end{array}$ & $\begin{array}{l}\text { Age-Adjusted } \\
\text { Rate per } 1,000 * *\end{array}$ & $\begin{array}{c}\text { Lower } 95 \% \\
\text { Confidence } \\
\text { Limit per } 1,000\end{array}$ & $\begin{array}{c}\text { Upper } 95 \% \\
\text { Confidence } \\
\text { Limit per 1,000 }\end{array}$ \\
\hline Diagnostic Category & ICD-9-CM Code & \multirow[b]{2}{*}{10} & \multirow[b]{2}{*}{2.1} & \multirow[b]{2}{*}{1.1} & \multirow[b]{2}{*}{4.1} \\
\hline -Dis of Veins, Lymphatics, Other & 451-459 & & & & \\
\hline RESPIRATORY SYSTEM & $460-519$ & 92 & 25.5 & 19.3 & 33.7 \\
\hline -Acute Respiratory Infections & $460-466$ & 21 & 5.5 & 3.2 & 9.5 \\
\hline -Other Dis Upper Respiratory Tract & $470-478$ & 36 & 9.3 & 6.1 & 14.4 \\
\hline -Pneumonia \& Influenza & $480-488$ & 14 & 3.9 & 2.0 & 7.9 \\
\hline -Chronic Obstructive Dis & $490-496$ & 20 & 6.5 & 3.5 & 12.3 \\
\hline -Other Respiratory Dis & $510-519$ & 1 & 0.2 & 0.0 & 1.5 \\
\hline DIGESTIVE SYSTEM & $520-579$ & 114 & 28.8 & 22.6 & 36.8 \\
\hline -Oral Cavity, Saliva Glands, Jaw & $520-529$ & 7 & 2.1 & 0.9 & 4.5 \\
\hline -Esophagus, Stomach, Duodenum & $530-538$ & 8 & 2.5 & 0.9 & 6.7 \\
\hline -Appendicitis & $540-543$ & 6 & 3.1 & 1.1 & 8.9 \\
\hline -Hernias & $550-553$ & 41 & 8.9 & 6.1 & 13.0 \\
\hline -Enteritis, Colitis & $555-558$ & 22 & 6.0 & 3.3 & 11.0 \\
\hline -Other Intestinal Dis & $560-569$ & 12 & 2.1 & 1.1 & 3.8 \\
\hline -Other Digestive Dis & $570-579$ & 18 & 4.2 & 2.6 & 6.8 \\
\hline GENITOURINARY SYSTEM & $580-629$ & 32 & 8.4 & 5.2 & 13.3 \\
\hline -Nephritis, Nephrosis & $580-589$ & 2 & 0.3 & 0.1 & 1.1 \\
\hline -Other Urinary Dis & $590-599$ & 20 & 6.3 & 3.5 & 11.3 \\
\hline -Male Genital Organ Dis & $600-608$ & 10 & 1.8 & 0.9 & 3.6 \\
\hline SKIN AND SUBCUTANEOUS TISSUE & $680-709$ & 14 & 3.4 & 2.0 & 5.9 \\
\hline -Infections & $680-686$ & 4 & 1.0 & 0.3 & 2.7 \\
\hline -Other Inflammatory Conditions & $690-698$ & 2 & 0.5 & 0.1 & 2.0 \\
\hline -Other & 700-709 & 8 & 1.9 & 0.9 & 4.0 \\
\hline $\begin{array}{l}\text { MUSCULOSKELETAL \& CONNECTIVE } \\
\text { TISSUE }\end{array}$ & $710-739$ & 130 & 31.5 & 25.2 & 39.3 \\
\hline -Arthropathies & $710-719$ & 48 & 11.4 & 7.8 & 16.5 \\
\hline -Dorsopathies & $720-724$ & 43 & 9.0 & 6.5 & 12.5 \\
\hline -Rheumatism, Excluding Back & 725-729 & 30 & 8.3 & 5.1 & 13.4 \\
\hline -Other Dis \& Acquired Deformities & 730-739 & 9 & 2.8 & 1.2 & 7.0 \\
\hline CONGENITAL ANOMALIES & $740-759$ & 1 & 0.3 & 0.0 & 1.8 \\
\hline $\begin{array}{l}\text { SYMPTOMS, SIGNS, \& ILL-DEFINED } \\
\text { CONDITIONS }\end{array}$ & $780-799$ & 51 & 11.9 & 8.6 & 16.5 \\
\hline -Symptoms & 780-789 & 46 & 10.8 & 7.7 & 15.3 \\
\hline -Non-Specific Abnormal Findings & $790-796$ & 5 & 1.0 & 0.4 & 2.6 \\
\hline INJURY \& POISONING & $800-999$ & 164 & 39.8 & 32.8 & 48.3 \\
\hline -Fracture - Skull & $800-804$ & 20 & 4.4 & 2.8 & 7.0 \\
\hline
\end{tabular}

(Continued)

* Only those diagnostic categories with at least one occurrence appear in this table.

**Standardized to age distribution of 2000 U.S. population. 
Oak Ridge National Laboratory 2010

Absence Data

Appendix K. Age-Adjusted Illness and Injury Rates by Diagnostic Category*

Part 1. Men

\begin{tabular}{|c|c|c|c|c|c|}
\hline & & $\begin{array}{l}\text { Number of } \\
\text { Diagnoses }\end{array}$ & $\begin{array}{c}\text { Age-Adjusted } \\
\text { Rate per } 1,000 * *\end{array}$ & $\begin{array}{c}\text { Lower } 95 \% \\
\text { Confidence } \\
\text { Limit per 1,000 }\end{array}$ & $\begin{array}{c}\text { Upper } 95 \% \\
\text { Confidence } \\
\text { Limit per 1,000 }\end{array}$ \\
\hline Diagnostic Category & ICD-9-CM Code & \multirow[b]{2}{*}{9} & \multirow[b]{2}{*}{2.0} & \multirow[b]{2}{*}{1.0} & \multirow[b]{2}{*}{4.1} \\
\hline -Fracture - Upper Limb & $810-819$ & & & & \\
\hline -Fracture - Lower Limb & $820-829$ & 14 & 3.1 & 1.8 & 5.4 \\
\hline -Dislocation & $830-839$ & 27 & 6.3 & 3.9 & 10.3 \\
\hline -Sprains \& Strains - Back & 846-847 & 9 & 3.1 & 1.3 & 7.2 \\
\hline -Sprains \& Strains - Other & $840-845,848$ & 42 & 12.6 & 8.3 & 19.1 \\
\hline -Intracranial Injury & $850-854$ & 2 & 0.4 & 0.1 & 1.6 \\
\hline -Internal Injury - Thorax, Abdomen, Pelvis & $860-869$ & 3 & 0.4 & 0.1 & 1.3 \\
\hline -Open Wound - Head, Neck, Trunk & $870-879$ & 4 & 0.7 & 0.3 & 2.0 \\
\hline -Superficial Injury & 910-919 & 6 & 1.2 & 0.5 & 2.8 \\
\hline -Contusion & $920-924$ & 8 & 1.4 & 0.7 & 2.8 \\
\hline -Burns & $940-949$ & 4 & 1.0 & 0.4 & 2.7 \\
\hline -Injury to Nerves \& Spinal Cord & $950-957$ & 1 & 0.1 & 0.0 & 1.0 \\
\hline -Complications of Surgical/Medical Care & 996-999 & 15 & 3.0 & 1.8 & 5.2 \\
\hline Total & & 782 & 188.5 & 172.2 & 206.4 \\
\hline
\end{tabular}

*Only those diagnostic categories with at least one occurrence appear in this table.

**Standardized to age distribution of 2000 U.S. population. 
Oak Ridge National Laboratory 2010

Absence Data

Appendix K. Age-Adjusted Illness and Injury Rates by Diagnostic Category*

Part 2. Women

\begin{tabular}{|c|c|c|c|c|c|}
\hline & & $\begin{array}{l}\text { Number of } \\
\text { Diagnoses }\end{array}$ & $\begin{array}{c}\text { Age-Adjusted } \\
\text { Rate per } 1,000 * *\end{array}$ & \begin{tabular}{c|} 
Lower $95 \%$ \\
Confidence \\
Limit per 1,000
\end{tabular} & $\begin{array}{c}\text { Upper 95\% } \\
\text { Confidence } \\
\text { Limit per 1,000 }\end{array}$ \\
\hline Diagnostic Category & ICD-9-CM Code & & & & \\
\hline INFECTIOUS \& PARASITIC DISEASES (DIS) & 001-139 & 19 & 15.6 & 8.9 & 27.2 \\
\hline -Intestinal Infectious Dis & 001-009 & 3 & 2.6 & 0.8 & 8.3 \\
\hline -Other Bacterial Dis & 030-041 & 7 & 6.8 & 2.8 & 16.6 \\
\hline -Viral Dis with Exanthem & 050-059 & 2 & 0.8 & 0.2 & 3.4 \\
\hline -Other Viral Dis \& Chlamydiae & 070-079 & 6 & 5.1 & 1.8 & 14.4 \\
\hline -Rickettsioses \& Other Arthropod & 080-088 & 1 & 0.3 & 0.0 & 2.1 \\
\hline MALIGNANT NEOPLASMS & $\begin{array}{l}140-208 \\
209.0-209.3 \\
230-234\end{array}$ & 13 & 5.5 & 3.0 & 10.3 \\
\hline -Digestive \& Peritoneal & 150-159 & 1 & 0.5 & 0.1 & 3.7 \\
\hline -Bone, Connective Tissue, Skin & $170-173,176$ & 2 & 0.6 & 0.1 & 2.3 \\
\hline -Breast & 174-175 & 3 & 1.3 & 0.4 & 4.3 \\
\hline -Genitourinary & 179-189 & 1 & 0.3 & 0.0 & 2.1 \\
\hline -Endocrine & \begin{tabular}{|l|}
$193-194$, \\
$209.0-209.3$
\end{tabular} & 1 & 0.3 & 0.0 & 2.1 \\
\hline -Other \& Unspecified Sites & $195-199,209.7$ & 4 & 1.4 & 0.5 & 3.9 \\
\hline -Lymphatic \& Hematopoietic & $200-208$ & 1 & 1.1 & 0.2 & 8.0 \\
\hline BENIGN \& UNCERTAIN NEOPLASMS & \begin{tabular}{|l|}
$209.4-209.6$ \\
$210-229,235-239$
\end{tabular} & 11 & 5.5 & 3.0 & 10.2 \\
\hline ENDOCRINE/METABOLIC/IMMUNITY & $240-279$ & 7 & 3.2 & 1.4 & 7.1 \\
\hline -Thyroid Gland Disorders & $240-246$ & 3 & 1.1 & 0.3 & 3.6 \\
\hline -Other Metabolic \& Immunity Disorders & $270-279$ & 4 & 2.1 & 0.7 & 6.1 \\
\hline BLOOD \& BLOOD-FORMING ORGANS & $280-289$ & 2 & 1.9 & 0.5 & 7.7 \\
\hline MENTAL DISORDERS & $290-319$ & 9 & 6.2 & 2.9 & 13.2 \\
\hline -Non-Psychotic Disorders & $300-302,306-316$ & 9 & 6.2 & 2.9 & 13.2 \\
\hline NERVOUS SYSTEM (NS) \& SENSE ORGANS & 320-389 & 41 & 24.6 & 17.0 & 35.7 \\
\hline -Hereditary/Degenerative Central NS Dis & 330-337 & 3 & 2.6 & 0.7 & 8.9 \\
\hline -Other Disorders of Central NS & $340-349$ & 4 & 3.2 & 0.7 & 13.6 \\
\hline -Disorders of Peripheral NS & $350-359$ & 8 & 3.9 & 1.7 & 8.7 \\
\hline -Disorders of Eye & $360-379$ & 12 & 5.1 & 2.7 & 9.5 \\
\hline -Diseases of Ear \& Mastoid & $380-389$ & 14 & 10.0 & 5.6 & 17.6 \\
\hline CIRCULATORY SYSTEM & $390-459$ & 13 & 7.6 & 4.2 & 13.8 \\
\hline -Hypertensive Dis & $401-405$ & 3 & 2.2 & 0.6 & 7.6 \\
\hline -Ischemic Heart Dis & $410-414$ & 4 & 2.5 & 0.9 & 7.1 \\
\hline -Other Heart Dis & $420-429$ & 2 & 1.5 & 0.4 & 6.0 \\
\hline -Cerebrovascular Dis & $430-438$ & 3 & 1.1 & 0.3 & 3.6 \\
\hline -Dis of Veins, Lymphatics, Other & 451-459 & 1 & 0.3 & 0.0 & 2.1 \\
\hline
\end{tabular}

(Continued)

* Only those diagnostic categories with at least one occurrence appear in this table.

**Standardized to age distribution of 2000 U.S. population. 
Oak Ridge National Laboratory 2010

Absence Data

Appendix K. Age-Adjusted Illness and Injury Rates by Diagnostic Category*

Part 2. Women

\begin{tabular}{|c|c|c|c|c|c|}
\hline & & $\begin{array}{l}\text { Number of } \\
\text { Diagnoses }\end{array}$ & $\begin{array}{l}\text { Age-Adjusted } \\
\text { Rate per } 1,000 * *\end{array}$ & $\begin{array}{c}\text { Lower } 95 \% \\
\text { Confidence } \\
\text { Limit per } 1,000\end{array}$ & $\begin{array}{c}\text { Upper } 95 \% \\
\text { Confidence } \\
\text { Limit per } 1,000\end{array}$ \\
\hline Diagnostic Category & ICD-9-CM Code & \multirow[b]{2}{*}{121} & \multirow[b]{2}{*}{79.0} & \multirow[b]{2}{*}{63.0} & \multirow[b]{2}{*}{99.1} \\
\hline RESPIRATORY SYSTEM & $460-519$ & & & & \\
\hline -Acute Respiratory Infections & $460-466$ & 37 & 26.4 & 17.1 & 40.6 \\
\hline -Other Dis Upper Respiratory Tract & $470-478$ & 46 & 31.3 & 22.0 & 44.4 \\
\hline -Pneumonia \& Influenza & $480-488$ & 17 & 9.9 & 5.7 & 17.1 \\
\hline -Chronic Obstructive Dis & $490-496$ & 21 & 11.4 & 6.5 & 20.1 \\
\hline DIGESTIVE SYSTEM & $520-579$ & 73 & 44.1 & 33.0 & 58.7 \\
\hline -Oral Cavity, Saliva Glands, Jaw & $520-529$ & 12 & 8.4 & 3.8 & 19.0 \\
\hline -Esophagus, Stomach, Duodenum & $530-538$ & 6 & 3.9 & 1.5 & 9.8 \\
\hline -Appendicitis & $540-543$ & 1 & 0.3 & 0.0 & 2.1 \\
\hline -Hernias & $550-553$ & 3 & 1.1 & 0.3 & 3.6 \\
\hline -Enteritis, Colitis & $555-558$ & 27 & 16.6 & 10.4 & 26.5 \\
\hline -Other Intestinal Dis & $560-569$ & 12 & 7.4 & 3.9 & 14.0 \\
\hline -Other Digestive Dis & $570-579$ & 12 & 6.3 & 3.3 & 12.1 \\
\hline GENITOURINARY SYSTEM & $580-629$ & 40 & 26.0 & 18.5 & 36.7 \\
\hline -Nephritis, Nephrosis & $580-589$ & 1 & 0.7 & 0.1 & 5.3 \\
\hline -Other Urinary Dis & $590-599$ & 5 & 2.8 & 1.0 & 7.5 \\
\hline -Breast Disorders & $610-612$ & 5 & 3.4 & 1.2 & 9.2 \\
\hline -Pelvic Inflammatory Dis & 614-616 & 4 & 3.5 & 1.3 & 9.8 \\
\hline -Other Female Disorders & $617-629$ & 25 & 15.6 & 10.2 & 24.0 \\
\hline SKIN AND SUBCUTANEOUS TISSUE & $680-709$ & 8 & 3.5 & 1.7 & 7.1 \\
\hline -Infections & $680-686$ & 2 & 0.8 & 0.2 & 3.4 \\
\hline -Other Inflammatory Conditions & $690-698$ & 2 & 1.0 & 0.3 & 4.2 \\
\hline -Other & 700-709 & 4 & 1.6 & 0.6 & 4.5 \\
\hline $\begin{array}{l}\text { MUSCULOSKELETAL \& CONNECTIVE } \\
\text { TISSUE }\end{array}$ & $710-739$ & 86 & 48.0 & 37.4 & 61.4 \\
\hline -Arthropathies & $710-719$ & 34 & 18.1 & 12.3 & 26.4 \\
\hline -Dorsopathies & $720-724$ & 32 & 19.8 & 12.9 & 30.3 \\
\hline -Rheumatism, Excluding Back & 725-729 & 15 & 7.5 & 4.3 & 13.2 \\
\hline -Other Dis \& Acquired Deformities & 730-739 & 5 & 2.6 & 1.1 & 6.5 \\
\hline CONGENITAL ANOMALIES & $740-759$ & 1 & 1.1 & 0.2 & 8.0 \\
\hline $\begin{array}{l}\text { SYMPTOMS, SIGNS, \& ILL-DEFINED } \\
\text { CONDITIONS }\end{array}$ & 780-799 & 33 & 21.0 & 14.2 & 31.0 \\
\hline -Symptoms & 780-789 & 30 & 18.8 & 12.5 & 28.4 \\
\hline -Non-Specific Abnormal Findings & $790-796$ & 3 & 2.2 & 0.6 & 7.6 \\
\hline INJURY \& POISONING & $800-999$ & 67 & 40.6 & 29.4 & 56.0 \\
\hline -Fracture - Skull & $800-804$ & 7 & 9.8 & 4.1 & 23.2 \\
\hline
\end{tabular}

(Continued)

* Only those diagnostic categories with at least one occurrence appear in this table.

**Standardized to age distribution of 2000 U.S. population. 
Oak Ridge National Laboratory 2010

Absence Data

Appendix K. Age-Adjusted Illness and Injury Rates by Diagnostic Category*

Part 2. Women

\begin{tabular}{|c|c|c|c|c|c|}
\hline & & $\begin{array}{l}\text { Number of } \\
\text { Diagnoses }\end{array}$ & $\begin{array}{c}\text { Age-Adjusted } \\
\text { Rate per } 1,000 * *\end{array}$ & $\begin{array}{c}\text { Lower } 95 \% \\
\text { Confidence } \\
\text { Limit per 1,000 }\end{array}$ & $\begin{array}{c}\text { Upper } 95 \% \\
\text { Confidence } \\
\text { Limit per 1,000 }\end{array}$ \\
\hline Diagnostic Category & ICD-9-CM Code & \multirow[b]{2}{*}{4} & \multirow[b]{2}{*}{2.1} & \multirow[b]{2}{*}{0.7} & \multirow[b]{2}{*}{5.8} \\
\hline -Fracture - Upper Limb & 810-819 & & & & \\
\hline -Fracture - Lower Limb & $820-829$ & 9 & 4.4 & 2.1 & 9.3 \\
\hline -Dislocation & $830-839$ & 12 & 3.7 & 2.1 & 6.6 \\
\hline -Sprains \& Strains - Back & 846-847 & 6 & 4.7 & 1.6 & 13.6 \\
\hline -Sprains \& Strains - Other & $840-845,848$ & 13 & 6.2 & 3.2 & 11.7 \\
\hline -Open Wound - Head, Neck, Trunk & $870-879$ & 3 & 3.6 & 0.9 & 13.9 \\
\hline -Late Effects of Accident & 905-909 & 1 & 0.3 & 0.0 & 2.1 \\
\hline -Contusion & $920-924$ & 4 & 1.2 & 0.4 & 3.1 \\
\hline -Unspecified Effects - External Causes & $990-995$ & 1 & 0.5 & 0.1 & 3.7 \\
\hline -Complications of Surgical/Medical Care & 996-999 & 7 & 4.3 & 1.9 & 9.6 \\
\hline Total & & 544 & 333.3 & 300.5 & 369.6 \\
\hline
\end{tabular}

* Only those diagnostic categories with at least one occurrence appear in this table.

**Standardized to age distribution of 2000 U.S. population. 
Oak Ridge National Laboratory 2010

Absence Data

Appendix K. Age-Adjusted Illness and Injury Rates by Diagnostic Category*

Part 3. Men and Women

\begin{tabular}{|c|c|c|c|c|c|}
\hline & & $\begin{array}{l}\text { Number of } \\
\text { Diagnoses }\end{array}$ & $\begin{array}{c}\text { Age-Adjusted } \\
\text { Rate per } 1,000 * *\end{array}$ & $\begin{array}{c}\text { Lower } 95 \% \\
\text { Confidence } \\
\text { Limit per } 1,000\end{array}$ & $\begin{array}{c}\text { Upper } 95 \% \\
\text { Confidence } \\
\text { Limit per 1,000 }\end{array}$ \\
\hline Diagnostic Category & ICD-9-CM Code & \multirow[b]{2}{*}{45} & \multirow[b]{2}{*}{10.7} & \multirow[b]{2}{*}{7.3} & \multirow[b]{2}{*}{15.9} \\
\hline INFECTIOUS \& PARASITIC DISEASES (DIS) & 001-139 & & & & \\
\hline -Intestinal Infectious Dis & 001-009 & 4 & 1.4 & 0.4 & 4.5 \\
\hline -Other Bacterial Dis & 030-041 & 18 & 4.4 & 2.4 & 7.9 \\
\hline -Viral Dis with Exanthem & 050-059 & 5 & 0.7 & 0.3 & 1.7 \\
\hline -Other Viral Dis \& Chlamydiae & 070-079 & 17 & 4.2 & 2.2 & 8.2 \\
\hline -Rickettsioses \& Other Arthropod & 080-088 & 1 & 0.1 & 0.0 & 0.7 \\
\hline MALIGNANT NEOPLASMS & $\begin{array}{l}140-208 \\
209.0-209.3 \\
230-234\end{array}$ & 20 & 2.7 & 1.7 & 4.3 \\
\hline -Lip, Oral Cavity, Pharynx & 140-149 & 1 & 0.2 & 0.0 & 1.2 \\
\hline -Digestive \& Peritoneal & 150-159 & 1 & 0.2 & 0.0 & 1.2 \\
\hline -Bone, Connective Tissue, Skin & $170-173,176$ & 2 & 0.2 & 0.0 & 0.7 \\
\hline -Breast & 174-175 & 3 & 0.4 & 0.1 & 1.4 \\
\hline -Genitourinary & 179-189 & 7 & 0.9 & 0.4 & 2.0 \\
\hline -Endocrine & $\begin{array}{l}\text { 193-194, } \\
209.0-209.3\end{array}$ & 1 & 0.1 & 0.0 & 0.7 \\
\hline -Other \& Unspecified Sites & 195-199, 209.7 & 4 & 0.4 & 0.2 & 1.2 \\
\hline -Lymphatic \& Hematopoietic & $200-208$ & 1 & 0.3 & 0.0 & 1.9 \\
\hline BENIGN \& UNCERTAIN NEOPLASMS & $\begin{array}{l}209.4-209.6 \\
210-229,235-239\end{array}$ & 27 & 3.7 & 2.5 & 5.6 \\
\hline ENDOCRINE/METABOLIC/IMMUNITY & $240-279$ & 17 & 2.1 & 1.3 & 3.6 \\
\hline -Thyroid Gland Disorders & $240-246$ & 4 & 0.5 & 0.2 & 1.5 \\
\hline -Other Endocrine Gland Dis & $249-259$ & 3 & 0.3 & 0.1 & 0.9 \\
\hline -Other Metabolic \& Immunity Disorders & $270-279$ & 10 & 1.3 & 0.7 & 2.6 \\
\hline BLOOD \& BLOOD-FORMING ORGANS & $280-289$ & 2 & 0.4 & 0.1 & 1.8 \\
\hline MENTAL DISORDERS & $290-319$ & 23 & 3.8 & 2.5 & 6.0 \\
\hline -Psychoses & $290-299$ & 1 & 0.2 & 0.0 & 1.2 \\
\hline -Non-Psychotic Disorders & $300-302,306-316$ & 16 & 2.4 & 1.4 & 4.2 \\
\hline -Alcohol Dependence & 303 & 2 & 0.3 & 0.1 & 1.4 \\
\hline -Drug Dependence & $304-305$ & 4 & 0.9 & 0.3 & 2.4 \\
\hline NERVOUS SYSTEM (NS) \& SENSE ORGANS & 320-389 & 90 & 13.5 & 10.7 & 17.2 \\
\hline -Organic Sleep Disorders & 327 & 3 & 0.7 & 0.2 & 2.3 \\
\hline -Hereditary/Degenerative Central NS Dis & 330-337 & 4 & 0.7 & 0.2 & 2.2 \\
\hline -Other Headache Syndromes & 339 & 1 & 0.1 & 0.0 & 0.7 \\
\hline -Other Disorders of Central NS & $340-349$ & 6 & 1.4 & 0.4 & 4.3 \\
\hline -Disorders of Peripheral NS & $350-359$ & 22 & 2.9 & 1.9 & 4.6 \\
\hline -Disorders of Eye & $360-379$ & 26 & 3.3 & 2.2 & 4.9 \\
\hline
\end{tabular}

(Continued)

* Only those diagnostic categories with at least one occurrence appear in this table.

**Standardized to age distribution of 2000 U.S. population. 
Oak Ridge National Laboratory 2010

Absence Data

Appendix K. Age-Adjusted Illness and Injury Rates by Diagnostic Category*

Part 3. Men and Women

\begin{tabular}{|c|c|c|c|c|c|}
\hline & & $\begin{array}{l}\text { Number of } \\
\text { Diagnoses }\end{array}$ & $\begin{array}{c}\text { Age-Adjusted } \\
\text { Rate per } 1,000^{* *}\end{array}$ & $\begin{array}{c}\text { Lower } 95 \% \\
\text { Confidence } \\
\text { Limit per } 1,000\end{array}$ & $\begin{array}{c}\text { Upper 95\% } \\
\text { Confidence } \\
\text { Limit per 1,000 }\end{array}$ \\
\hline Diagnostic Category & ICD-9-CM Code & \multirow[b]{2}{*}{28} & \multirow[b]{2}{*}{4.4} & \multirow[b]{2}{*}{2.9} & \multirow[b]{2}{*}{6.6} \\
\hline -Diseases of Ear \& Mastoid & 380-389 & & & & \\
\hline CIRCULATORY SYSTEM & $390-459$ & 75 & 10.1 & 7.9 & 12.9 \\
\hline -Hypertensive Dis & $401-405$ & 10 & 1.4 & 0.7 & 2.7 \\
\hline -Ischemic Heart Dis & $410-414$ & 20 & 2.4 & 1.5 & 3.9 \\
\hline -Dis of Pulmonary Circulation & 415-417 & 4 & 0.4 & 0.2 & 1.2 \\
\hline -Other Heart Dis & $420-429$ & 21 & 2.8 & 1.8 & 4.4 \\
\hline -Cerebrovascular Dis & $430-438$ & 6 & 0.8 & 0.3 & 1.8 \\
\hline -Dis of Arteries \& Capillaries & $440-449$ & 3 & 0.6 & 0.2 & 1.9 \\
\hline -Dis of Veins, Lymphatics, Other & 451-459 & 11 & 1.6 & 0.8 & 3.1 \\
\hline RESPIRATORY SYSTEM & $460-519$ & 213 & 40.8 & 34.2 & 48.7 \\
\hline -Acute Respiratory Infections & $460-466$ & 58 & 11.7 & 8.3 & 16.5 \\
\hline -Other Dis Upper Respiratory Tract & $470-478$ & 82 & 15.6 & 11.9 & 20.4 \\
\hline -Pneumonia \& Influenza & $480-488$ & 31 & 5.5 & 3.6 & 8.5 \\
\hline -Chronic Obstructive Dis & $490-496$ & 41 & 7.9 & 5.1 & 12.1 \\
\hline -Other Respiratory Dis & $510-519$ & 1 & 0.2 & 0.0 & 1.2 \\
\hline DIGESTIVE SYSTEM & $520-579$ & 187 & 33.3 & 27.7 & 40.1 \\
\hline -Oral Cavity, Saliva Glands, Jaw & $520-529$ & 19 & 4.3 & 2.4 & 7.8 \\
\hline -Esophagus, Stomach, Duodenum & $530-538$ & 14 & 2.7 & 1.3 & 5.4 \\
\hline -Appendicitis & $540-543$ & 7 & 2.2 & 0.8 & 6.0 \\
\hline -Hernias & $550-553$ & 44 & 6.6 & 4.6 & 9.5 \\
\hline -Enteritis, Colitis & $555-558$ & 49 & 9.1 & 6.3 & 13.1 \\
\hline -Other Intestinal Dis & $560-569$ & 24 & 3.5 & 2.3 & 5.5 \\
\hline -Other Digestive Dis & $570-579$ & 30 & 4.9 & 3.3 & 7.1 \\
\hline GENITOURINARY SYSTEM & $580-629$ & 72 & 12.9 & 9.8 & 17.0 \\
\hline -Nephritis, Nephrosis & $580-589$ & 3 & 0.4 & 0.1 & 1.1 \\
\hline -Other Urinary Dis & 590-599 & 25 & 5.1 & 3.1 & 8.5 \\
\hline -Male Genital Organ Dis & $600-608$ & 10 & 1.3 & 0.7 & 2.6 \\
\hline -Breast Disorders & $610-612$ & 5 & 0.9 & 0.3 & 2.4 \\
\hline -Pelvic Inflammatory Dis & $614-616$ & 4 & 0.9 & 0.3 & 2.4 \\
\hline -Other Female Disorders & $617-629$ & 25 & 4.4 & 2.9 & 6.6 \\
\hline SKIN AND SUBCUTANEOUS TISSUE & $680-709$ & 22 & 3.6 & 2.3 & 5.6 \\
\hline -Infections & $680-686$ & 6 & 1.0 & 0.4 & 2.3 \\
\hline -Other Inflammatory Conditions & $690-698$ & 4 & 0.7 & 0.3 & 1.8 \\
\hline -Other & 700-709 & 12 & 2.0 & 1.1 & 3.6 \\
\hline $\begin{array}{l}\text { MUSCULOSKELETAL \& CONNECTIVE } \\
\text { TISSUE }\end{array}$ & $710-739$ & 216 & 35.7 & 30.3 & 42.1 \\
\hline
\end{tabular}

(Continued)

*Only those diagnostic categories with at least one occurrence appear in this table.

**Standardized to age distribution of 2000 U.S. population. 
Oak Ridge National Laboratory 2010

Absence Data

Appendix K. Age-Adjusted Illness and Injury Rates by Diagnostic Category*

Part 3. Men and Women

\begin{tabular}{|c|c|c|c|c|c|}
\hline & & $\begin{array}{l}\text { Number of } \\
\text { Diagnoses }\end{array}$ & $\begin{array}{c}\text { Age-Adjusted } \\
\text { Rate per } 1,000 * *\end{array}$ & \begin{tabular}{|c|} 
Lower $95 \%$ \\
Confidence \\
Limit per 1,000
\end{tabular} & $\begin{array}{c}\text { Upper } 95 \% \\
\text { Confidence } \\
\text { Limit per 1,000 }\end{array}$ \\
\hline Diagnostic Category & ICD-9-CM Code & \multirow[b]{2}{*}{82} & \multirow[b]{2}{*}{12.9} & \multirow[b]{2}{*}{9.9} & \multirow[b]{2}{*}{16.8} \\
\hline -Arthropathies & $710-719$ & & & & \\
\hline -Dorsopathies & $720-724$ & 75 & 12.0 & 9.2 & 15.6 \\
\hline -Rheumatism, Excluding Back & 725-729 & 45 & 8.0 & 5.6 & 11.7 \\
\hline -Other Dis \& Acquired Deformities & 730-739 & 14 & 2.8 & 1.4 & 5.5 \\
\hline CONGENITAL ANOMALIES & $740-759$ & 2 & 0.4 & 0.1 & 1.8 \\
\hline $\begin{array}{l}\text { SYMPTOMS, SIGNS, \& ILL-DEFINED } \\
\text { CONDITIONS }\end{array}$ & 780-799 & 84 & 14.0 & 11.0 & 17.9 \\
\hline -Symptoms & 780-789 & 76 & 12.7 & 9.8 & 16.5 \\
\hline -Non-Specific Abnormal Findings & $790-796$ & 8 & 1.3 & 0.6 & 2.7 \\
\hline INJURY \& POISONING & $800-999$ & 231 & 40.5 & 34.3 & 47.8 \\
\hline -Fracture - Skull & 800-804 & 27 & 6.1 & 3.7 & 10.1 \\
\hline -Fracture - Upper Limb & 810-819 & 13 & 2.1 & 1.2 & 3.7 \\
\hline -Fracture - Lower Limb & 820-829 & 23 & 3.5 & 2.3 & 5.4 \\
\hline -Dislocation & 830-839 & 39 & 5.7 & 3.8 & 8.5 \\
\hline -Sprains \& Strains - Back & 846-847 & 15 & 3.7 & 1.9 & 7.1 \\
\hline -Sprains \& Strains - Other & $840-845,848$ & 55 & 10.6 & 7.5 & 15.1 \\
\hline -Intracranial Injury & $850-854$ & 2 & 0.3 & 0.1 & 1.1 \\
\hline -Internal Injury - Thorax, Abdomen, Pelvis & $860-869$ & 3 & 0.3 & 0.1 & 0.9 \\
\hline -Open Wound - Head, Neck, Trunk & 870-879 & 7 & 1.6 & 0.6 & 4.4 \\
\hline -Late Effects of Accident & 905-909 & 1 & 0.1 & 0.0 & 0.7 \\
\hline -Superficial Injury & 910-919 & 6 & 0.8 & 0.3 & 2.0 \\
\hline -Contusion & 920-924 & 12 & 1.3 & 0.7 & 2.4 \\
\hline -Burns & 940-949 & 4 & 0.7 & 0.3 & 1.8 \\
\hline -Injury to Nerves \& Spinal Cord & $950-957$ & 1 & 0.1 & 0.0 & 0.7 \\
\hline -Unspecified Effects - External Causes & $990-995$ & 1 & 0.2 & 0.0 & 1.2 \\
\hline -Complications of Surgical/Medical Care & 996-999 & 22 & 3.4 & 2.2 & 5.3 \\
\hline Total & & 1326 & 228.6 & 213.7 & 244.6 \\
\hline
\end{tabular}

* Only those diagnostic categories with at least one occurrence appear in this table.

**Standardized to age distribution of 2000 U.S. population. 
Oak Ridge National Laboratory 2010

OSHA Data

Appendix L. Number of Workers with at Least One OSHA Event by Gender, Age, and Job Category*

\begin{tabular}{|c|c|c|c|c|c|c|c|c|c|}
\hline \multirow{3}{*}{ Job Category } & \multicolumn{4}{|c|}{ Women } & \multicolumn{4}{|c|}{ Men } & \multirow[b]{3}{*}{ TOTAL } \\
\hline & \multicolumn{3}{|c|}{ Age Group } & \multirow[b]{2}{*}{ TOTAL } & \multicolumn{3}{|c|}{ Age Group } & \multirow[b]{2}{*}{ TOTAL } & \\
\hline & $30-39$ & $40-49$ & $50+$ & & $30-39$ & $40-49$ & $50+$ & & \\
\hline Professional & 0 & 2 & 5 & 7 & 1 & 0 & 4 & 5 & 12 \\
\hline Administrative Support & 0 & 0 & 0 & 0 & 0 & 1 & 2 & 3 & 3 \\
\hline Technical Support & 1 & 2 & 3 & 6 & 2 & 0 & 3 & 5 & 11 \\
\hline Service & 0 & 1 & 1 & 2 & 1 & 1 & 0 & 2 & 4 \\
\hline Security and Fire & 0 & 0 & 0 & 0 & 1 & 0 & 0 & 1 & 1 \\
\hline Crafts & 0 & 0 & 0 & 0 & 2 & 0 & 8 & 10 & 10 \\
\hline Line Operators & 0 & 0 & 0 & 0 & 2 & 1 & 0 & 3 & 3 \\
\hline TOTAL & 1 & 5 & 9 & 15 & 9 & 3 & 17 & 29 & 44 \\
\hline
\end{tabular}

*Only those job categories and gender/age combinations with at least one OSHA event appear in this table.

Appendix M. Total Number of Workdays Lost or with Restricted Activity from OSHA Events by Gender and Age

\begin{tabular}{|c|c|c|c|c|c|c|c|c|c|}
\hline \multirow[b]{2}{*}{ Age Group } & \multicolumn{3}{|c|}{ Women } & \multicolumn{3}{|c|}{ Men } & \multicolumn{3}{|c|}{ TOTAL } \\
\hline & $\begin{array}{l}\text { Number of } \\
\text { Events }\end{array}$ & $\begin{array}{c}\text { Days } \\
\text { Restricted }\end{array}$ & Days Lost & $\begin{array}{c}\text { Number of } \\
\text { Events }\end{array}$ & $\begin{array}{c}\text { Days } \\
\text { Restricted }\end{array}$ & Days Lost & $\begin{array}{c}\text { Number of } \\
\text { Events }\end{array}$ & $\begin{array}{c}\text { Days } \\
\text { Restricted }\end{array}$ & Days Lost \\
\hline $30-39$ & 1 & 0 & 0 & 9 & 0 & 218 & 10 & 0 & 218 \\
\hline $40-49$ & 5 & 0 & 0 & 3 & 0 & 87 & 8 & 0 & 87 \\
\hline $50+$ & 9 & 94 & 0 & 17 & 0 & 180 & 26 & 94 & 180 \\
\hline TOTAL & 15 & 94 & 0 & 29 & 0 & 485 & 44 & 94 & 485 \\
\hline
\end{tabular}

Appendix N. Total Number of Workdays Lost or with Restricted Activity from OSHA Events by Gender and Job Category*

\begin{tabular}{|c|c|c|c|c|c|c|c|c|c|}
\hline \multirow[b]{2}{*}{ Job Category } & \multicolumn{3}{|c|}{ Women } & \multicolumn{3}{|c|}{ Men } & \multicolumn{3}{|c|}{ TOTAL } \\
\hline & $\begin{array}{c}\text { Number of } \\
\text { Events }\end{array}$ & $\begin{array}{c}\text { Days } \\
\text { Restricted }\end{array}$ & Days Lost & $\begin{array}{c}\text { Number of } \\
\text { Events }\end{array}$ & $\begin{array}{c}\text { Days } \\
\text { Restricted }\end{array}$ & Days Lost & $\begin{array}{c}\text { Number of } \\
\text { Events }\end{array}$ & $\begin{array}{c}\text { Days } \\
\text { Restricted }\end{array}$ & Days Lost \\
\hline $\begin{array}{l}\text { Administrative } \\
\text { Support }\end{array}$ & 0 & 0 & 0 & 3 & 0 & 0 & 3 & 0 & 0 \\
\hline Security and Fire & 0 & 0 & 0 & 1 & 0 & 18 & 1 & 0 & 18 \\
\hline Crafts & 0 & 0 & 0 & 10 & 0 & 180 & 10 & 0 & 180 \\
\hline Line Operators & 0 & 0 & 0 & 3 & 0 & 180 & 3 & 0 & 180 \\
\hline TOTAL & 15 & 94 & 0 & 29 & 0 & 485 & 44 & 94 & 485 \\
\hline
\end{tabular}

*Only those job categories with at least one OSHA event appear in this table. 
Oak Ridge National Laboratory 2010

OSHA Data

Appendix O. Number of Diagnoses in Each Diagnostic Category by Gender and Age*

\begin{tabular}{|c|c|c|c|c|c|}
\hline & & \multicolumn{4}{|c|}{ Women } \\
\hline & & \multicolumn{3}{|c|}{ Age Group } & \multirow[b]{2}{*}{ TOTAL } \\
\hline & & 30 - 39 & $40-49$ & $50+$ & \\
\hline Diagnostic Category & ICD-9-CM code & \multirow[b]{2}{*}{0} & \multirow[b]{2}{*}{0} & \multirow[b]{2}{*}{0} & \multirow[b]{2}{*}{0} \\
\hline NERVOUS SYSTEM (NS) \& SENSE ORGANS & 320-389 & & & & \\
\hline -Disorders of Eye & $360-379$ & 0 & 0 & 0 & 0 \\
\hline -Diseases of Ear \& Mastoid & $380-389$ & 0 & 0 & 0 & 0 \\
\hline SKIN AND SUBCUTANEOUS TISSUE & $680-709$ & 0 & 0 & 1 & 1 \\
\hline -Other Inflammatory Conditions & $690-698$ & 0 & 0 & 1 & 1 \\
\hline $\begin{array}{l}\text { MUSCULOSKELETAL \& CONNECTIVE } \\
\text { TISSUE }\end{array}$ & $710-739$ & 0 & 3 & 4 & 7 \\
\hline -Arthropathies & $710-719$ & 0 & 1 & 3 & 4 \\
\hline -Dorsopathies & $720-724$ & 0 & 2 & 1 & 3 \\
\hline -Rheumatism, Excluding Back & 725-729 & 0 & 0 & 0 & 0 \\
\hline -Other Dis \& Acquired Deformities & $730-739$ & 0 & 0 & 0 & 0 \\
\hline $\begin{array}{l}\text { SYMPTOMS, SIGNS, \& ILL-DEFINED } \\
\text { CONDITIONS }\end{array}$ & $780-799$ & 0 & 0 & 1 & 1 \\
\hline -Symptoms & 780-789 & 0 & 0 & 1 & 1 \\
\hline INJURY \& POISONING & 800-999 & 1 & 6 & 12 & 19 \\
\hline -Fracture - Upper Limb & 810-819 & 0 & 1 & 1 & 2 \\
\hline -Fracture - Lower Limb & $820-829$ & 0 & 0 & 2 & 2 \\
\hline -Dislocation & $830-839$ & 0 & 0 & 0 & 0 \\
\hline -Sprains \& Strains - Back & 846-847 & 0 & 0 & 2 & 2 \\
\hline -Sprains \& Strains - Other & $840-845,848$ & 0 & 1 & 2 & 3 \\
\hline -Open Wound - Head, Neck, Trunk & $870-879$ & 0 & 0 & 0 & $\overline{0}$ \\
\hline -Open Wound - Upper Limb & $880-887$ & 1 & 1 & 1 & 3 \\
\hline -Superficial Injury & $910-919$ & 0 & 0 & 2 & 2 \\
\hline -Contusion & $920-924$ & 0 & 0 & 0 & $\overline{0}$ \\
\hline -Foreign Body Entering Orifice & $930-939$ & 0 & 0 & 0 & $\overline{0}$ \\
\hline -Complications \& Unspecified Injuries & 958-959 & 0 & 3 & 2 & 5 \\
\hline -Toxic Effects - Non-medicinal & 980-989 & 0 & 0 & 0 & 0 \\
\hline
\end{tabular}

*Only those diagnostic categories and gender/age combinations with at least one OSHA event appear in this table. 
Oak Ridge National Laboratory 2010

OSHA Data

Appendix O. Number of Diagnoses in Each Diagnostic Category by Gender and Age*

\begin{tabular}{|c|c|c|c|c|c|c|}
\hline & & \multicolumn{4}{|c|}{ Men } & \multirow[b]{3}{*}{ TOTAL } \\
\hline & & \multicolumn{3}{|c|}{ Age Group } & \multirow[b]{2}{*}{ TOTAL } & \\
\hline & & $30-39$ & $40-49$ & $50+$ & & \\
\hline Diagnostic Category & ICD-9-CM code & & & & & \\
\hline NERVOUS SYSTEM (NS) \& SENSE ORGANS & $320-389$ & 0 & 0 & 2 & 2 & 2 \\
\hline -Disorders of Eye & $360-379$ & 0 & 0 & 1 & 1 & 1 \\
\hline -Diseases of Ear \& Mastoid & $380-389$ & 0 & 0 & 1 & 1 & 1 \\
\hline SKIN AND SUBCUTANEOUS TISSUE & 680-709 & 0 & 0 & 0 & 0 & 1 \\
\hline -Other Inflammatory Conditions & $690-698$ & 0 & 0 & 0 & 0 & 1 \\
\hline $\begin{array}{l}\text { MUSCULOSKELETAL \& CONNECTIVE } \\
\text { TISSUE }\end{array}$ & $710-739$ & 4 & 3 & 9 & 16 & 23 \\
\hline -Arthropathies & $710-719$ & 3 & 1 & 3 & 7 & 11 \\
\hline -Dorsopathies & $720-724$ & 1 & 0 & 4 & 5 & 8 \\
\hline -Rheumatism, Excluding Back & $725-729$ & 0 & 2 & 1 & 3 & 3 \\
\hline -Other Dis \& Acquired Deformities & $730-739$ & 0 & 0 & 1 & 1 & 1 \\
\hline $\begin{array}{l}\text { SYMPTOMS, SIGNS, \& ILL-DEFINED } \\
\text { CONDITIONS }\end{array}$ & $780-799$ & 0 & 1 & 0 & 1 & 2 \\
\hline -Symptoms & $780-789$ & 0 & 1 & 0 & 1 & 2 \\
\hline INJURY \& POISONING & $800-999$ & 9 & 4 & 16 & 29 & 48 \\
\hline -Fracture - Upper Limb & 810-819 & 0 & 0 & 1 & 1 & 3 \\
\hline -Fracture - Lower Limb & $820-829$ & 0 & 0 & 0 & 0 & 2 \\
\hline -Dislocation & $830-839$ & 0 & 0 & 1 & 1 & 1 \\
\hline -Sprains \& Strains - Back & 846-847 & 2 & 0 & 2 & 4 & 6 \\
\hline -Sprains \& Strains - Other & $840-845,848$ & 1 & 0 & 2 & 3 & 6 \\
\hline -Open Wound - Head, Neck, Trunk & $870-879$ & 1 & 0 & 2 & 3 & 3 \\
\hline -Open Wound - Upper Limb & $880-887$ & 2 & 1 & 1 & 4 & 7 \\
\hline -Superficial Injury & 910-919 & 0 & 0 & 1 & 1 & 3 \\
\hline -Contusion & $920-924$ & 1 & 0 & 3 & 4 & 4 \\
\hline -Foreign Body Entering Orifice & 930-939 & 0 & 0 & 2 & 2 & 2 \\
\hline -Complications \& Unspecified Injuries & 958-959 & 1 & 2 & 1 & 4 & 9 \\
\hline -Toxic Effects - Non-medicinal & $980-989$ & 1 & 1 & 0 & 2 & 2 \\
\hline
\end{tabular}

\begin{tabular}{|c|c|c|c|c|c|c|c|c|c|}
\hline & \multicolumn{4}{|c|}{ Women } & \multicolumn{4}{|c|}{ Men } & \multirow[b]{3}{*}{ TOTAL } \\
\hline & \multicolumn{3}{|c|}{ Age Group } & \multirow[b]{2}{*}{ TOTAL } & \multicolumn{3}{|c|}{ Age Group } & \multirow[b]{2}{*}{ TOTAL } & \\
\hline & \begin{tabular}{|l|}
$30-39$ \\
\end{tabular} & $40-49$ & $50+$ & & $30-39$ & $40-49$ & $50+$ & & \\
\hline Diagnostic Category & \multirow[b]{2}{*}{1} & \multirow[b]{2}{*}{9} & \multirow[b]{2}{*}{18} & \multirow[b]{2}{*}{28} & \multirow[b]{2}{*}{13} & \multirow[b]{2}{*}{8} & \multirow[b]{2}{*}{27} & \multirow[b]{2}{*}{48} & \\
\hline Total & & & & & & & & & 76 \\
\hline
\end{tabular}

*Only those diagnostic categories and gender/age combinations with at least one OSHA event appear in this table. 
Oak Ridge National Laboratory 2010

OSHA Data

Appendix P. Number of Workdays Lost or with Restricted Activity in Each Diagnostic Category by Gender and Age*

\begin{tabular}{|c|c|c|c|c|c|c|c|}
\hline & & \multicolumn{6}{|c|}{ Women } \\
\hline & & \multicolumn{6}{|c|}{ Age Group } \\
\hline & & \multicolumn{2}{|c|}{$30-39$} & \multicolumn{2}{|c|}{$40-49$} & \multicolumn{2}{|c|}{$50+$} \\
\hline & & $\begin{array}{c}\text { Days } \\
\text { Restricted }\end{array}$ & Days Lost & \begin{tabular}{|c|} 
Days \\
Restricted
\end{tabular} & Days Lost & \begin{tabular}{|c|} 
Days \\
Restricted
\end{tabular} & Days Lost \\
\hline Diagnostic Category & ICD-9-CM Codes & \multirow[b]{2}{*}{0} & \multirow[b]{2}{*}{0} & \multirow[b]{2}{*}{0} & \multirow[b]{2}{*}{0} & \multirow[b]{2}{*}{0} & \multirow[b]{2}{*}{0} \\
\hline -Other Inflammatory Conditions & $690-698$ & & & & & & \\
\hline -Arthropathies & $710-719$ & 0 & 0 & 0 & 0 & 0 & 0 \\
\hline -Dorsopathies & $720-724$ & 0 & 0 & 0 & 0 & 0 & 0 \\
\hline -Symptoms & $780-789$ & 0 & 0 & 0 & 0 & 0 & 0 \\
\hline -Fracture - Upper Limb & $810-819$ & 0 & 0 & 0 & 0 & 0 & 0 \\
\hline -Fracture - Lower Limb & $820-829$ & 0 & 0 & 0 & 0 & 94 & 0 \\
\hline -Sprains \& Strains - Back & $846-847$ & 0 & 0 & 0 & 0 & 0 & 0 \\
\hline -Sprains \& Strains - Other & $840-845,848$ & 0 & 0 & 0 & 0 & 0 & 0 \\
\hline -Open Wound - Upper Limb & $880-887$ & 0 & 0 & 0 & 0 & 0 & 0 \\
\hline -Superficial Injury & $910-919$ & 0 & 0 & 0 & 0 & 0 & 0 \\
\hline $\begin{array}{l}\text {-Complications \& Unspecified } \\
\text { Injuries }\end{array}$ & 958-959 & 0 & 0 & 0 & 0 & 0 & 0 \\
\hline
\end{tabular}

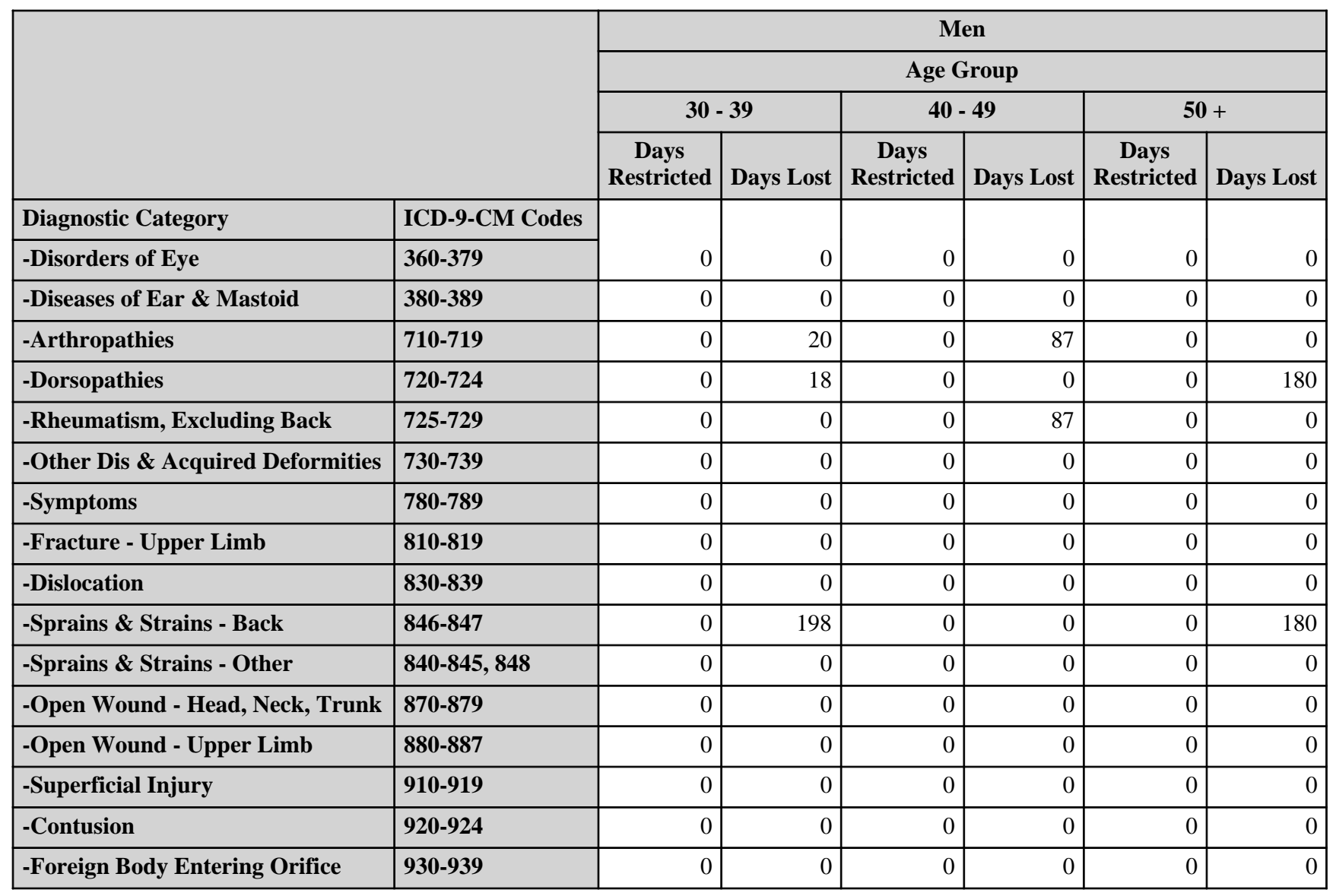

(Continued)

*OSHA events with >1 ICD-9-CM code in the same diagnostic category were counted only once. Only those diagnostic categories and gender/age combinations with at least one occurrence appear in this table. 
Oak Ridge National Laboratory 2010

OSHA Data

Appendix P. Number of Workdays Lost or with Restricted Activity in Each Diagnostic Category by Gender and Age*

\begin{tabular}{|c|c|c|c|c|c|c|c|}
\hline & & \multicolumn{6}{|c|}{ Men } \\
\hline & & \multicolumn{6}{|c|}{ Age Group } \\
\hline & & \multicolumn{2}{|c|}{$30-39$} & \multicolumn{2}{|c|}{$40-49$} & \multicolumn{2}{|c|}{$50+$} \\
\hline & & \begin{tabular}{|c|} 
Days \\
Restricted
\end{tabular} & Days Lost & \begin{tabular}{|c} 
Days \\
Restricted
\end{tabular} & Days Lost & \begin{tabular}{|c} 
Days \\
Restricted
\end{tabular} & Days Lost \\
\hline Diagnostic Category & ICD-9-CM Codes & & & & & & \\
\hline $\begin{array}{l}\text {-Complications \& Unspecified } \\
\text { Injuries }\end{array}$ & 958-959 & 0 & 20 & 0 & 87 & 0 & 0 \\
\hline -Toxic Effects - Non-medicinal & 980-989 & 0 & 0 & 0 & 0 & 0 & 0 \\
\hline
\end{tabular}

*OSHA events with >1 ICD-9-CM code in the same diagnostic category were counted only once. Only those diagnostic categories and gender/age combinations with at least one occurrence appear in this table. 
Oak Ridge National Laboratory 2010

OSHA Data

Appendix Q. Number of Occurrences in Each Accident Category by Gender and Age*

\begin{tabular}{|c|c|c|c|c|c|c|c|c|c|c|}
\hline & & \multicolumn{4}{|c|}{ Women } & \multicolumn{4}{|c|}{ Men } & \multirow[b]{3}{*}{ TOTAL } \\
\hline & & \multicolumn{3}{|c|}{ Age Group } & \multirow[b]{2}{*}{ TOTAL } & \multicolumn{3}{|c|}{ Age Group } & \multirow[b]{2}{*}{ TOTAL } & \\
\hline & & 30 - 39 & $40-49$ & $\mathbf{5 0 +}$ & & $30-39$ & $40-49$ & $50+$ & & \\
\hline Surgical and Medical Procedures & E878-E879 & 0 & 0 & 0 & 0 & 0 & 0 & 1 & 1 & 1 \\
\hline Falls & E880-E888 & 0 & 1 & 4 & 5 & 0 & 0 & 2 & 2 & 7 \\
\hline $\begin{array}{l}\text { Submersion/Suffocation/Foreign } \\
\text { Bodies }\end{array}$ & E910-E915 & 0 & 0 & 0 & 0 & 0 & 0 & 2 & 2 & 2 \\
\hline Other Accidents & E916-E928 & 1 & 4 & 4 & 9 & 8 & 2 & 11 & 21 & 30 \\
\hline
\end{tabular}

*Only those accident types and gender/age combinations with at least one occurrence appear in this table. 
Oak Ridge National Laboratory 2010

OSHA Data

Appendix R. Number of Workdays Lost or with Restricted Activity in Each Accident Category by Gender and Age*

\begin{tabular}{|c|c|c|c|c|c|c|c|}
\hline & & \multicolumn{6}{|c|}{ Women } \\
\hline & & \multicolumn{6}{|c|}{ Age Group } \\
\hline & & \multicolumn{2}{|c|}{30 - 39} & \multicolumn{2}{|c|}{$40-49$} & \multicolumn{2}{|c|}{$50+$} \\
\hline & & $\begin{array}{c}\text { Days } \\
\text { Restricted }\end{array}$ & Days Lost & $\begin{array}{c}\text { Days } \\
\text { Restricted }\end{array}$ & Days Lost & $\begin{array}{c}\text { Days } \\
\text { Restricted }\end{array}$ & Days Lost \\
\hline Type of Accident & E Codes & \multirow[b]{2}{*}{0} & \multirow[b]{2}{*}{0} & \multirow[b]{2}{*}{0} & \multirow[b]{2}{*}{0} & \multirow[b]{2}{*}{94} & \multirow[b]{2}{*}{0} \\
\hline Falls & E880-E888 & & & & & & \\
\hline Other Accidents & E916-E928 & 0 & 0 & 0 & 0 & 0 & 0 \\
\hline
\end{tabular}

\begin{tabular}{|c|c|c|c|c|c|c|c|}
\hline & & \multicolumn{6}{|c|}{ Men } \\
\hline & & \multicolumn{6}{|c|}{ Age Group } \\
\hline & & \multicolumn{2}{|c|}{30 - 39} & \multicolumn{2}{|c|}{$40-49$} & \multicolumn{2}{|c|}{$50+$} \\
\hline & & $\begin{array}{c}\text { Days } \\
\text { Restricted }\end{array}$ & Days Lost & $\begin{array}{c}\text { Days } \\
\text { Restricted }\end{array}$ & Days Lost & $\begin{array}{c}\text { Days } \\
\text { Restricted }\end{array}$ & Days Lost \\
\hline Type of Accident & E Codes & \multirow[b]{2}{*}{0} & \multirow[b]{2}{*}{0} & \multirow[b]{2}{*}{0} & \multirow[b]{2}{*}{0} & \multirow[b]{2}{*}{0} & \multirow[b]{2}{*}{0} \\
\hline Surgical and Medical Procedures & E878-E879 & & & & & & \\
\hline Falls & E880-E888 & 0 & 0 & 0 & 0 & 0 & 0 \\
\hline Natural/Environmental Factors & E900-E909 & 0 & 0 & 0 & 0 & 0 & 0 \\
\hline $\begin{array}{l}\text { Submersion/Suffocation/Foreign } \\
\text { Bodies }\end{array}$ & E910-E915 & 0 & 0 & 0 & 0 & 0 & 0 \\
\hline Other Accidents & E916-E928 & 0 & 218 & 0 & 87 & 0 & 180 \\
\hline
\end{tabular}

*OSHA events with $>1 \mathrm{E}$ code in the same accident type were counted only once. Only those accident types and gender/age combinations with at least one occurrence appear in this table. 
Oak Ridge National Laboratory 2010

OSHA Data

Appendix S. Number of Diagnoses in Each Diagnostic Category by Gender and Job Category*

\begin{tabular}{|c|c|c|c|c|c|}
\hline & & \multicolumn{4}{|c|}{ Women } \\
\hline & & \multicolumn{3}{|c|}{ Job Category } & \multirow[b]{2}{*}{ TOTAL } \\
\hline & & Professional & Technical Support & Service & \\
\hline Diagnostic Category & ICD-9-CM Code & & & & \\
\hline $\begin{array}{l}\text { SKIN AND } \\
\text { SUBCUTANEOUS } \\
\text { TISSUE }\end{array}$ & $680-709$ & 0 & 0 & 1 & 1 \\
\hline $\begin{array}{l}\text {-Other Inflammatory } \\
\text { Conditions }\end{array}$ & 690-698 & 0 & 0 & 1 & 1 \\
\hline $\begin{array}{l}\text { MUSCULOSKELETAL } \\
\text { \& CONNECTIVE } \\
\text { TISSUE }\end{array}$ & $710-739$ & 5 & 2 & 0 & 7 \\
\hline -Arthropathies & $710-719$ & 3 & 1 & 0 & 4 \\
\hline -Dorsopathies & $720-724$ & 2 & 1 & 0 & 3 \\
\hline $\begin{array}{l}\text { SYMPTOMS, } \\
\text { SIGNS, \& ILL-DEFINED } \\
\text { CONDITIONS }\end{array}$ & $780-799$ & 0 & 1 & 0 & 1 \\
\hline -Symptoms & $780-789$ & 0 & 1 & 0 & 1 \\
\hline INJURY \& POISONING & $800-999$ & 11 & 7 & 1 & 19 \\
\hline -Fracture - Upper Limb & $810-819$ & 1 & 1 & 0 & 2 \\
\hline -Fracture - Lower Limb & $820-829$ & 1 & 1 & 0 & 2 \\
\hline -Sprains \& Strains - Back & 846-847 & 2 & 0 & 0 & 2 \\
\hline $\begin{array}{l}\text {-Sprains \& Strains - } \\
\text { Other }\end{array}$ & $840-845,848$ & 3 & 0 & 0 & 3 \\
\hline $\begin{array}{l}\text {-Open Wound - Upper } \\
\text { Limb }\end{array}$ & $880-887$ & 1 & 1 & 1 & 3 \\
\hline -Superficial Injury & $910-919$ & 2 & 0 & 0 & 2 \\
\hline $\begin{array}{l}\text {-Complications \& } \\
\text { Unspecified Injuries }\end{array}$ & 958-959 & 1 & 4 & 0 & 5 \\
\hline
\end{tabular}

\begin{tabular}{|c|c|c|c|c|}
\hline & \multicolumn{4}{|c|}{ Women } \\
\hline & \multicolumn{3}{|c|}{ Job Category } & \multirow[b]{2}{*}{ TOTAL } \\
\hline & Professional & Technical Support & Service & \\
\hline Diagnostic Category & \multirow[b]{2}{*}{16} & \multirow[b]{2}{*}{10} & \multirow[b]{2}{*}{2} & \multirow[b]{2}{*}{28} \\
\hline Total & & & & \\
\hline
\end{tabular}

*Only those diagnostic categories and gender/job category combinations with at least one occurrence appear in this table. 
Oak Ridge National Laboratory 2010

OSHA Data

Appendix S. Number of Diagnoses in Each Diagnostic Category by Gender and Job Category*

\begin{tabular}{|c|c|c|c|c|c|c|c|c|c|}
\hline & \multicolumn{8}{|c|}{ Men } \\
\hline & & \multicolumn{7}{|c|}{ Job Category } & \multirow[b]{2}{*}{ TOTAL } \\
\hline & & Professional & $\begin{array}{c}\text { Administrative } \\
\text { Support }\end{array}$ & \begin{tabular}{|c|} 
Technical \\
Support
\end{tabular} & Service & \begin{tabular}{l|} 
Security \\
and Fire
\end{tabular} & Crafts & \begin{tabular}{|c|} 
Line \\
Operators
\end{tabular} & \\
\hline Diagnostic Category & ICD-9-CM Code & & & & & & & & \\
\hline $\begin{array}{l}\text { NERVOUS SYSTEM } \\
\text { (NS) \& SENSE ORGANS }\end{array}$ & 320-389 & 0 & 0 & 0 & 0 & 0 & 2 & 0 & 2 \\
\hline -Disorders of Eye & $360-379$ & 0 & 0 & 0 & 0 & 0 & 1 & 0 & 1 \\
\hline $\begin{array}{l}\text {-Diseases of } \\
\text { Ear \& Mastoid }\end{array}$ & $380-389$ & 0 & 0 & 0 & 0 & 0 & 1 & 0 & 1 \\
\hline $\begin{array}{l}\text { MUSCULOSKELETAL } \\
\text { \& CONNECTIVE } \\
\text { TISSUE }\end{array}$ & \begin{tabular}{|l|}
$710-739$ \\
\end{tabular} & 3 & 2 & 3 & 2 & 1 & 4 & 1 & 16 \\
\hline -Arthropathies & $710-719$ & 1 & 0 & 2 & 1 & 0 & 2 & 1 & 7 \\
\hline -Dorsopathies & $720-724$ & 1 & 0 & 1 & 0 & 1 & 2 & 0 & 5 \\
\hline $\begin{array}{l}\text {-Rheumatism, Excluding } \\
\text { Back }\end{array}$ & $725-729$ & 0 & 2 & 0 & 1 & 0 & 0 & 0 & 3 \\
\hline $\begin{array}{l}\text {-Other Dis \& Acquired } \\
\text { Deformities }\end{array}$ & \begin{tabular}{|l|}
$730-739$ \\
\end{tabular} & 1 & 0 & 0 & 0 & 0 & 0 & 0 & 1 \\
\hline $\begin{array}{l}\text { SYMPTOMS, } \\
\text { SIGNS, \& ILL-DEFINED } \\
\text { CONDITIONS }\end{array}$ & \begin{tabular}{|l|}
$780-799$ \\
\end{tabular} & 0 & 1 & 0 & 0 & 0 & 0 & 0 & 1 \\
\hline -Symptoms & $780-789$ & 0 & 1 & 0 & 0 & 0 & 0 & 0 & 1 \\
\hline INJURY \& POISONING & $800-999$ & 5 & 2 & 5 & 3 & 1 & 10 & 3 & 29 \\
\hline -Fracture - Upper Limb & $810-819$ & 0 & 0 & 0 & 0 & 0 & 1 & 0 & 1 \\
\hline -Dislocation & $830-839$ & 0 & 0 & 0 & 0 & 0 & 1 & 0 & 1 \\
\hline -Sprains \& Strains - Back & $846-847$ & 1 & 0 & 0 & 0 & 1 & 1 & 1 & 4 \\
\hline $\begin{array}{l}\text {-Sprains \& Strains - } \\
\text { Other }\end{array}$ & $840-845,848$ & 1 & 0 & 1 & 0 & 0 & 1 & 0 & 3 \\
\hline $\begin{array}{l}\text {-Open Wound - Head, } \\
\text { Neck, Trunk }\end{array}$ & $870-879$ & 1 & 0 & 1 & 0 & 0 & 1 & 0 & 3 \\
\hline $\begin{array}{l}\text {-Open Wound - Upper } \\
\text { Limb }\end{array}$ & $880-887$ & 0 & 0 & 1 & 1 & 0 & 1 & 1 & 4 \\
\hline -Superficial Injury & 910-919 & 0 & 0 & 1 & 0 & 0 & 0 & 0 & 1 \\
\hline -Contusion & $920-924$ & 0 & 0 & 0 & 0 & 0 & 3 & 1 & 4 \\
\hline $\begin{array}{l}\text {-Foreign Body Entering } \\
\text { Orifice }\end{array}$ & 930-939 & 0 & 1 & 0 & 0 & 0 & 1 & 0 & 2 \\
\hline $\begin{array}{l}\text {-Complications \& } \\
\text { Unspecified Injuries }\end{array}$ & 958-959 & 2 & 0 & 0 & 2 & 0 & 0 & 0 & 4 \\
\hline $\begin{array}{l}\text {-Toxic } \\
\text { Effects - Non-medicinal }\end{array}$ & \begin{tabular}{|c|}
$980-989$ \\
\end{tabular} & 0 & 1 & 1 & 0 & 0 & 0 & 0 & 2 \\
\hline
\end{tabular}

*Only those diagnostic categories and gender/job category combinations with at least one occurrence appear in this table. 
Oak Ridge National Laboratory 2010

OSHA Data

Appendix S. Number of Diagnoses in Each Diagnostic Category by Gender and Job Category*

\begin{tabular}{|c|c|c|c|c|c|c|c|c|}
\hline & \multicolumn{8}{|c|}{ Men } \\
\hline & \multicolumn{7}{|c|}{ Job Category } & \multirow[b]{2}{*}{ TOTAL } \\
\hline & Professional & $\begin{array}{c}\text { Administrative } \\
\text { Support }\end{array}$ & $\begin{array}{c}\text { Technical } \\
\text { Support }\end{array}$ & Service & $\begin{array}{l}\text { Security and } \\
\text { Fire }\end{array}$ & Crafts & $\begin{array}{c}\text { Line } \\
\text { Operators }\end{array}$ & \\
\hline Diagnostic Category & \multirow[b]{2}{*}{8} & \multirow[b]{2}{*}{5} & \multirow[b]{2}{*}{8} & \multirow[b]{2}{*}{5} & \multirow[b]{2}{*}{2} & \multirow[b]{2}{*}{16} & \multirow[b]{2}{*}{4} & \multirow[b]{2}{*}{48} \\
\hline Total & & & & & & & & \\
\hline
\end{tabular}

*Only those diagnostic categories and gender/job category combinations with at least one occurrence appear in this table. 
Oak Ridge National Laboratory 2010

OSHA Data

Appendix T. Number of Workdays Lost or with Restricted Activity in Each Diagnostic Category by Gender and Job Category*

\begin{tabular}{|c|c|c|c|c|c|c|c|}
\hline & & \multicolumn{6}{|c|}{ Women } \\
\hline & & \multicolumn{6}{|c|}{ Job Category } \\
\hline & & \multicolumn{2}{|c|}{ Professional } & \multicolumn{2}{|c|}{ Technical Support } & \multicolumn{2}{|c|}{ Service } \\
\hline & & $\begin{array}{c}\text { Days } \\
\text { Restricted }\end{array}$ & Days Lost & \begin{tabular}{|c|} 
Days \\
Restricted
\end{tabular} & Days Lost & \begin{tabular}{|c|} 
Days \\
Restricted
\end{tabular} & Days Lost \\
\hline Diagnostic Category & ICD-9-CM Codes & & & & & & \\
\hline -Other Inflammatory Conditions & $690-698$ & 0 & 0 & 0 & 0 & 0 & 0 \\
\hline -Arthropathies & $710-719$ & 0 & 0 & 0 & 0 & 0 & 0 \\
\hline -Dorsopathies & $720-724$ & 0 & 0 & 0 & 0 & 0 & 0 \\
\hline -Symptoms & \begin{tabular}{|l|}
$780-789$ \\
\end{tabular} & 0 & 0 & 0 & 0 & 0 & 0 \\
\hline -Fracture - Upper Limb & $810-819$ & 0 & 0 & 0 & 0 & 0 & 0 \\
\hline -Fracture - Lower Limb & 820-829 & 0 & 0 & 94 & 0 & 0 & 0 \\
\hline -Sprains \& Strains - Back & $846-847$ & 0 & 0 & 0 & 0 & 0 & 0 \\
\hline -Sprains \& Strains - Other & $840-845,848$ & 0 & 0 & 0 & 0 & 0 & 0 \\
\hline -Open Wound - Upper Limb & $880-887$ & 0 & 0 & 0 & 0 & 0 & 0 \\
\hline -Superficial Injury & $910-919$ & 0 & 0 & 0 & 0 & 0 & 0 \\
\hline $\begin{array}{l}\text {-Complications \& Unspecified } \\
\text { Injuries }\end{array}$ & 958-959 & 0 & 0 & 0 & 0 & 0 & 0 \\
\hline
\end{tabular}

*OSHA events with >1 ICD-9-CM code in the same diagnostic category were counted only once. Only those diagnostic categories and gender/job category combinations with at least one occurrence appear in this table. 
Oak Ridge National Laboratory 2010

OSHA Data

Appendix T. Number of Workdays Lost or with Restricted Activity in Each Diagnostic Category by Gender and Job Category*

\begin{tabular}{|c|c|c|c|c|c|c|c|c|c|}
\hline & & \multicolumn{8}{|c|}{ Men } \\
\hline & & \multicolumn{8}{|c|}{ Job Category } \\
\hline & & \multicolumn{2}{|c|}{ Professional } & \multicolumn{2}{|c|}{$\begin{array}{l}\text { Administrative } \\
\text { Support }\end{array}$} & \multicolumn{2}{|c|}{$\begin{array}{l}\text { Technical } \\
\text { Support }\end{array}$} & \multicolumn{2}{|c|}{ Service } \\
\hline & & $\begin{array}{c}\text { Days } \\
\text { Restricted }\end{array}$ & $\begin{array}{l}\text { Days } \\
\text { Lost }\end{array}$ & $\begin{array}{c}\text { Days } \\
\text { Restricted }\end{array}$ & $\begin{array}{l}\text { Days } \\
\text { Lost }\end{array}$ & \begin{tabular}{c|} 
Days \\
Restricted
\end{tabular} & \begin{tabular}{l|} 
Days \\
Lost
\end{tabular} & $\begin{array}{c}\text { Days } \\
\text { Restricted }\end{array}$ & $\begin{array}{l}\text { Days } \\
\text { Lost }\end{array}$ \\
\hline Diagnostic Category & ICD-9-CM Codes & & & & & & & & \\
\hline -Disorders of Eye & $360-379$ & 0 & 0 & 0 & 0 & 0 & 0 & 0 & 0 \\
\hline -Diseases of Ear \& Mastoid & 380-389 & 0 & 0 & 0 & 0 & 0 & 0 & 0 & 0 \\
\hline -Arthropathies & $710-719$ & 0 & 20 & 0 & 0 & 0 & 0 & 0 & 87 \\
\hline -Dorsopathies & $720-724$ & 0 & 0 & 0 & 0 & 0 & 0 & 0 & 0 \\
\hline -Rheumatism, Excluding Back & $725-729$ & 0 & 0 & 0 & 0 & 0 & 0 & 0 & 87 \\
\hline -Other Dis \& Acquired Deformities & $730-739$ & 0 & 0 & 0 & 0 & 0 & 0 & 0 & 0 \\
\hline -Symptoms & 780-789 & 0 & 0 & 0 & 0 & 0 & 0 & 0 & 0 \\
\hline -Fracture - Upper Limb & 810-819 & 0 & 0 & 0 & 0 & 0 & 0 & 0 & 0 \\
\hline -Dislocation & 830-839 & 0 & 0 & 0 & 0 & 0 & 0 & 0 & 0 \\
\hline -Sprains \& Strains - Back & 846-847 & 0 & 0 & 0 & 0 & 0 & 0 & 0 & 0 \\
\hline -Sprains \& Strains - Other & $840-845,848$ & 0 & 0 & 0 & 0 & 0 & 0 & 0 & 0 \\
\hline -Open Wound - Head, Neck, Trunk & 870-879 & 0 & 0 & 0 & 0 & 0 & 0 & 0 & 0 \\
\hline -Open Wound - Upper Limb & $880-887$ & 0 & 0 & 0 & 0 & 0 & 0 & 0 & 0 \\
\hline -Superficial Injury & $910-919$ & 0 & 0 & 0 & 0 & 0 & 0 & 0 & 0 \\
\hline -Contusion & $920-924$ & 0 & 0 & 0 & 0 & 0 & 0 & 0 & 0 \\
\hline -Foreign Body Entering Orifice & $930-939$ & 0 & 0 & 0 & 0 & 0 & 0 & 0 & 0 \\
\hline $\begin{array}{l}\text {-Complications \& Unspecified } \\
\text { Injuries }\end{array}$ & 958-959 & 0 & 20 & 0 & 0 & 0 & 0 & 0 & 87 \\
\hline -Toxic Effects - Non-medicinal & $980-989$ & 0 & 0 & 0 & 0 & 0 & 0 & 0 & 0 \\
\hline
\end{tabular}

*OSHA events with >1 ICD-9-CM code in the same diagnostic category were counted only once. Only those diagnostic categories and gender/job category combinations with at least one occurrence appear in this table. 
Oak Ridge National Laboratory 2010

OSHA Data

Appendix T. Number of Workdays Lost or with Restricted Activity in Each Diagnostic Category by Gender and Job Category*

\begin{tabular}{|c|c|c|c|c|c|c|c|}
\hline & \multirow{2}{*}{\multicolumn{6}{|c|}{$\begin{array}{c}\text { Men } \\
\text { Job Category }\end{array}$}} \\
\hline & & & & & & & \\
\hline & & \multicolumn{2}{|c|}{ Security and Fire } & \multicolumn{2}{|c|}{ Crafts } & \multicolumn{2}{|c|}{ Line Operators } \\
\hline & & $\begin{array}{c}\text { Days } \\
\text { Restricted }\end{array}$ & $\begin{array}{l}\text { Days } \\
\text { Lost }\end{array}$ & \begin{tabular}{|c|} 
Days \\
Restricted
\end{tabular} & \begin{tabular}{c|} 
Days \\
Lost
\end{tabular} & $\begin{array}{c}\text { Days } \\
\text { Restricted }\end{array}$ & $\begin{array}{l}\text { Days } \\
\text { Lost }\end{array}$ \\
\hline Diagnostic Category & ICD-9-CM Codes & \multirow[b]{2}{*}{0} & \multirow[b]{2}{*}{0} & \multirow[b]{2}{*}{0} & \multirow[b]{2}{*}{0} & \multirow[b]{2}{*}{0} & \multirow[b]{2}{*}{0} \\
\hline -Disorders of Eye & $360-379$ & & & & & & \\
\hline -Diseases of Ear \& Mastoid & 380-389 & 0 & 0 & 0 & 0 & 0 & 0 \\
\hline -Arthropathies & $710-719$ & 0 & 0 & 0 & 0 & 0 & 0 \\
\hline -Dorsopathies & $720-724$ & 0 & 18 & 0 & 180 & 0 & 0 \\
\hline -Rheumatism, Excluding Back & 725-729 & 0 & 0 & 0 & 0 & 0 & 0 \\
\hline -Other Dis \& Acquired Deformities & 730-739 & 0 & 0 & 0 & 0 & 0 & 0 \\
\hline -Symptoms & $780-789$ & 0 & 0 & 0 & 0 & 0 & 0 \\
\hline -Fracture - Upper Limb & 810-819 & 0 & 0 & 0 & 0 & 0 & 0 \\
\hline -Dislocation & \begin{tabular}{|l|}
$830-839$ \\
\end{tabular} & 0 & 0 & 0 & 0 & 0 & 0 \\
\hline -Sprains \& Strains - Back & 846-847 & 0 & 18 & 0 & 180 & 0 & 180 \\
\hline -Sprains \& Strains - Other & $840-845,848$ & 0 & 0 & 0 & 0 & 0 & 0 \\
\hline -Open Wound - Head, Neck, Trunk & 870-879 & 0 & 0 & 0 & 0 & 0 & 0 \\
\hline -Open Wound - Upper Limb & 880-887 & 0 & 0 & 0 & 0 & 0 & 0 \\
\hline -Superficial Injury & 910-919 & 0 & 0 & 0 & 0 & 0 & 0 \\
\hline -Contusion & $920-924$ & 0 & 0 & 0 & 0 & 0 & 0 \\
\hline -Foreign Body Entering Orifice & $930-939$ & 0 & 0 & 0 & 0 & 0 & 0 \\
\hline $\begin{array}{l}\text {-Complications \& Unspecified } \\
\text { Injuries }\end{array}$ & 958-959 & 0 & 0 & 0 & 0 & 0 & 0 \\
\hline -Toxic Effects - Non-medicinal & $980-989$ & 0 & 0 & 0 & 0 & 0 & 0 \\
\hline
\end{tabular}

*OSHA events with >1 ICD-9-CM code in the same diagnostic category were counted only once. Only those diagnostic categories and gender/job category combinations with at least one occurrence appear in this table. 
Oak Ridge National Laboratory 2010

OSHA Data

Appendix U. Number of Occurrences in Each Accident Category by Gender and Job Category*

\begin{tabular}{|c|c|c|c|c|c|}
\hline & & \multicolumn{4}{|c|}{ Women } \\
\hline & & \multicolumn{3}{|c|}{ Job Category } & \multirow[b]{2}{*}{ TOTAL } \\
\hline & & Professional & \begin{tabular}{|c|} 
Technical \\
Support
\end{tabular} & Service & \\
\hline Type of Accident & E CODES & \multirow[b]{2}{*}{4} & \multirow[b]{2}{*}{1} & \multirow[b]{2}{*}{0} & \multirow[b]{2}{*}{5} \\
\hline Falls & E880-E888 & & & & \\
\hline Other Accidents & E916-E928 & 3 & 5 & 1 & 9 \\
\hline
\end{tabular}

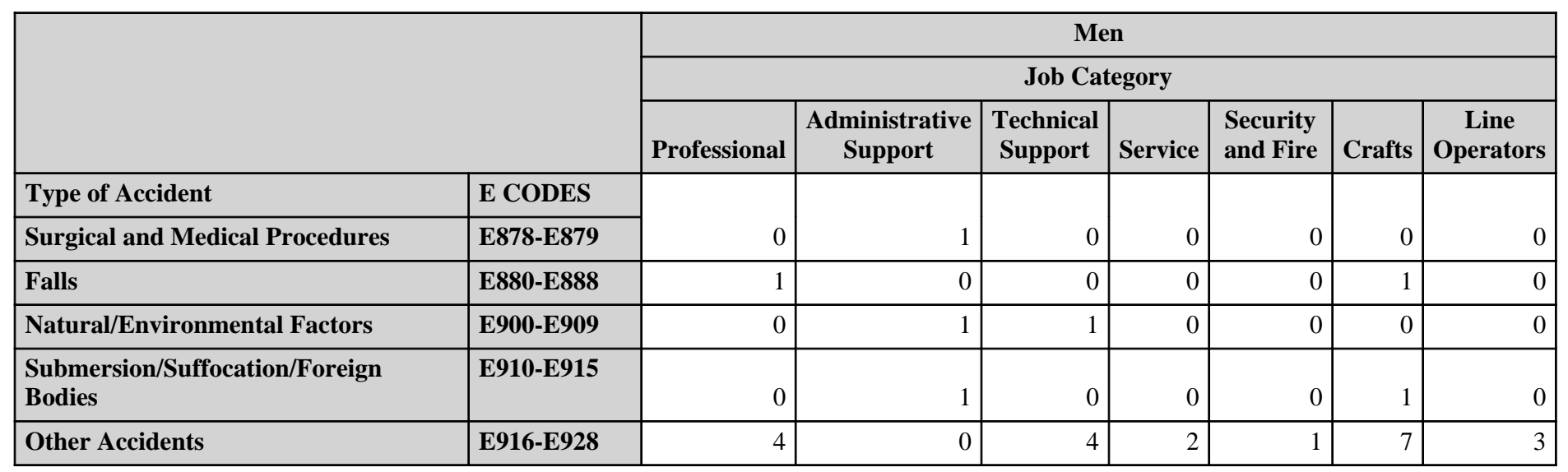

\begin{tabular}{|l|l|r|}
\hline \multicolumn{2}{|l|}{} & Men \\
& & \\
Type of Accident & E CODES & \\
\hline Surgical and Medical Procedures & E878-E879 & 1 \\
\hline Falls & E880-E888 & 2 \\
\hline Natural/Environmental Factors & E900-E909 & 2 \\
\hline $\begin{array}{l}\text { Submersion/Suffocation/Foreign } \\
\text { Bodies }\end{array}$ & E910-E915 & 2 \\
\hline Other Accidents & E916-E928 & 21 \\
\hline
\end{tabular}

*Only those accident types and gender/job category combinations with at least one occurrence appear in this table. 
Oak Ridge National Laboratory 2010

OSHA Data

Appendix V. Number of Workdays Lost or with Restricted Activity in Each Accident Category by Gender and Job Category*

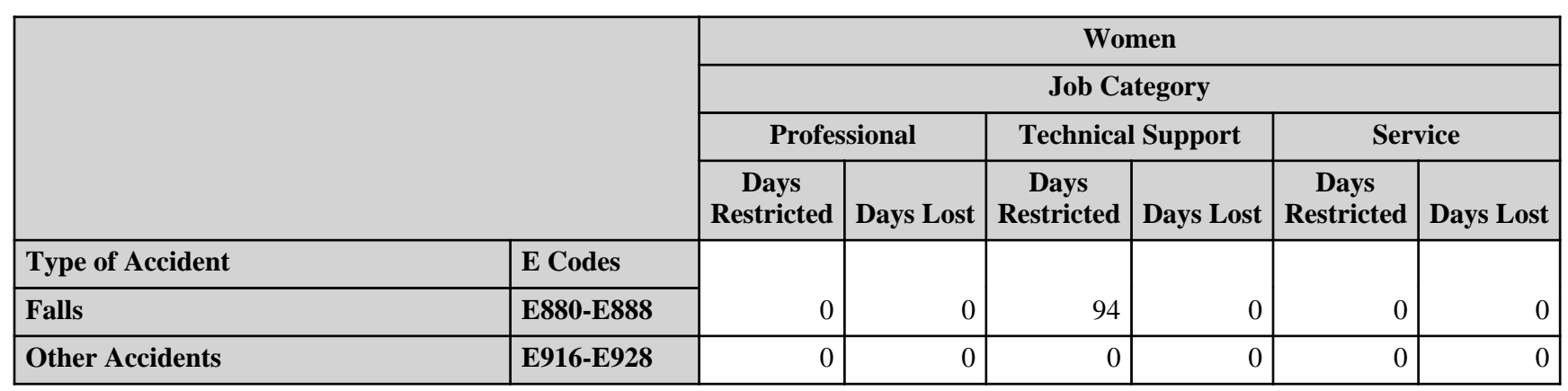

\begin{tabular}{|c|c|c|c|c|c|c|c|c|c|}
\hline & \multicolumn{8}{|c|}{ Men } \\
\hline & & \multicolumn{8}{|c|}{ Job Category } \\
\hline & & \multicolumn{2}{|c|}{ Professional } & \multicolumn{2}{|c|}{$\begin{array}{l}\text { Administrative } \\
\text { Support }\end{array}$} & \multicolumn{2}{|c|}{$\begin{array}{l}\text { Technical } \\
\text { Support }\end{array}$} & \multicolumn{2}{|c|}{ Service } \\
\hline & & \begin{tabular}{|c|} 
Days \\
Restricted
\end{tabular} & $\begin{array}{l}\text { Days } \\
\text { Lost }\end{array}$ & \begin{tabular}{|c|} 
Days \\
Restricted
\end{tabular} & $\begin{array}{l}\text { Days } \\
\text { Lost }\end{array}$ & \begin{tabular}{|c|} 
Days \\
Restricted
\end{tabular} & $\begin{array}{l}\text { Days } \\
\text { Lost }\end{array}$ & \begin{tabular}{|c|} 
Days \\
Restricted
\end{tabular} & $\begin{array}{l}\text { Days } \\
\text { Lost }\end{array}$ \\
\hline Type of Accident & E Codes & & & & & & & & \\
\hline Surgical and Medical Procedures & E878-E879 & 0 & 0 & 0 & 0 & 0 & 0 & 0 & 0 \\
\hline Falls & E880-E888 & 0 & 0 & 0 & 0 & 0 & 0 & 0 & 0 \\
\hline Natural/Environmental Factors & E900-E909 & 0 & 0 & 0 & 0 & 0 & 0 & 0 & 0 \\
\hline $\begin{array}{l}\text { Submersion/Suffocation/Foreign } \\
\text { Bodies }\end{array}$ & E910-E915 & 0 & 0 & 0 & 0 & 0 & 0 & 0 & 0 \\
\hline Other Accidents & E916-E928 & 0 & 20 & 0 & 0 & 0 & 0 & 0 & 87 \\
\hline
\end{tabular}

\begin{tabular}{|c|c|c|c|c|c|c|c|}
\hline & \multicolumn{6}{|c|}{ Men } \\
\hline & & \multicolumn{6}{|c|}{ Job Category } \\
\hline & & \multicolumn{2}{|c|}{ Security and Fire } & \multicolumn{2}{|c|}{ Crafts } & \multicolumn{2}{|c|}{ Line Operators } \\
\hline & & \begin{tabular}{c|} 
Days \\
Restricted
\end{tabular} & $\begin{array}{l}\text { Days } \\
\text { Lost }\end{array}$ & \begin{tabular}{|c|} 
Days \\
Restricted
\end{tabular} & $\begin{array}{l}\text { Days } \\
\text { Lost }\end{array}$ & \begin{tabular}{|c|} 
Days \\
Restricted
\end{tabular} & $\begin{array}{l}\text { Days } \\
\text { Lost }\end{array}$ \\
\hline Type of Accident & E Codes & & & & & & \\
\hline Surgical and Medical Procedures & E878-E879 & 0 & 0 & 0 & 0 & 0 & 0 \\
\hline Falls & E880-E888 & 0 & 0 & 0 & 0 & 0 & 0 \\
\hline Natural/Environmental Factors & E900-E909 & 0 & 0 & 0 & 0 & 0 & 0 \\
\hline $\begin{array}{l}\text { Submersion/Suffocation/Foreign } \\
\text { Bodies }\end{array}$ & E910-E915 & 0 & 0 & 0 & 0 & 0 & 0 \\
\hline Other Accidents & \begin{tabular}{|l|} 
E916-E928 \\
\end{tabular} & 0 & 18 & 0 & 180 & 0 & 180 \\
\hline
\end{tabular}

*OSHA events with $>1 \mathrm{E}$ code in the same accident type were counted only once. Only those accident types and gender/job category combinations with at least one occurrence appear in this table. 
Oak Ridge National Laboratory 2010

OSHA Data

Appendix W. Age-Adjusted OSHA Illness and Injury Rates by Diagnostic Category*

Part 1. Men

\begin{tabular}{|c|c|c|c|c|c|}
\hline & & $\begin{array}{l}\text { Number of } \\
\text { Diagnoses }\end{array}$ & $\begin{array}{c}\text { Age-Adjusted } \\
\text { Rate per } 1,000 * *\end{array}$ & \begin{tabular}{|c|} 
Lower $95 \%$ \\
Confidence \\
Limit per 1,000
\end{tabular} & $\begin{array}{c}\text { Upper 95\% } \\
\text { Confidence } \\
\text { Limit per 1,000 }\end{array}$ \\
\hline Diagnostic Category & ICD-9-CM Code & \multirow[b]{2}{*}{2} & \multirow[b]{2}{*}{0.3} & \multirow[b]{2}{*}{0.1} & \multirow[b]{2}{*}{1.1} \\
\hline NERVOUS SYSTEM (NS) \& SENSE ORGANS & $320-389$ & & & & \\
\hline -Disorders of Eye & $360-379$ & 1 & 0.1 & 0.0 & $\overline{1.0}$ \\
\hline -Diseases of Ear \& Mastoid & 380-389 & 1 & 0.1 & 0.0 & 1.0 \\
\hline $\begin{array}{l}\text { MUSCULOSKELETAL \& CONNECTIVE } \\
\text { TISSUE }\end{array}$ & $710-739$ & 16 & 3.5 & 2.0 & 5.9 \\
\hline -Arthropathies & 710-719 & 7 & 1.7 & 0.8 & 3.9 \\
\hline -Dorsopathies & $720-724$ & 5 & 1.0 & 0.4 & 2.5 \\
\hline -Rheumatism, Excluding Back & $725-729$ & 3 & 0.6 & 0.2 & 2.1 \\
\hline -Other Dis \& Acquired Deformities & $730-739$ & 1 & 0.1 & 0.0 & 1.0 \\
\hline $\begin{array}{l}\text { SYMPTOMS, SIGNS, \& ILL-DEFINED } \\
\text { CONDITIONS }\end{array}$ & 780-799 & 1 & 0.3 & 0.0 & 1.8 \\
\hline -Symptoms & 780-789 & 1 & 0.3 & 0.0 & 1.8 \\
\hline INJURY \& POISONING & $800-999$ & 29 & 6.9 & 4.7 & 10.2 \\
\hline -Fracture - Upper Limb & $810-819$ & 1 & 0.1 & 0.0 & 1.0 \\
\hline -Dislocation & 830-839 & 1 & 0.2 & 0.0 & 1.5 \\
\hline -Sprains \& Strains - Back & 846-847 & 4 & 1.1 & 0.4 & 3.0 \\
\hline -Sprains \& Strains - Other & $840-845,848$ & 3 & 0.6 & 0.2 & 2.2 \\
\hline -Open Wound - Head, Neck, Trunk & $870-879$ & 3 & 0.7 & 0.2 & 2.4 \\
\hline -Open Wound - Upper Limb & 880-887 & 4 & 1.2 & 0.4 & 3.2 \\
\hline -Superficial Injury & $910-919$ & 1 & 0.2 & 0.0 & 1.5 \\
\hline -Contusion & $920-924$ & 4 & 0.9 & 0.3 & 2.6 \\
\hline -Foreign Body Entering Orifice & 930-939 & 2 & 0.3 & 0.1 & 1.1 \\
\hline -Toxic Effects - Non-medicinal & 980-989 & 2 & 0.6 & 0.1 & 2.5 \\
\hline -Complications of Surgical/Medical Care & 996-999 & 4 & 1.0 & 0.4 & 2.8 \\
\hline Total & & 48 & 10.9 & 8.0 & 14.8 \\
\hline
\end{tabular}

* Only those diagnostic categories with at least one occurrence appear in this table.

**Standardized to age distribution of 2000 U.S. population. 
Oak Ridge National Laboratory 2010

OSHA Data

Appendix W. Age-Adjusted OSHA Illness and Injury Rates by Diagnostic Category*

Part 2. Women

\begin{tabular}{|c|c|c|c|c|c|}
\hline & & $\begin{array}{l}\text { Number of } \\
\text { Diagnoses }\end{array}$ & $\begin{array}{l}\text { Age-Adjusted } \\
\text { Rate per } 1,000 * *\end{array}$ & \begin{tabular}{|c|} 
Lower $95 \%$ \\
Confidence \\
Limit per 1,000
\end{tabular} & $\begin{array}{c}\text { Upper } 95 \% \\
\text { Confidence } \\
\text { Limit per } 1,000\end{array}$ \\
\hline Diagnostic Category & ICD-9-CM Code & \multirow[b]{2}{*}{1} & \multirow[b]{2}{*}{0.3} & \multirow[b]{2}{*}{0.0} & \multirow[b]{2}{*}{2.1} \\
\hline SKIN AND SUBCUTANEOUS TISSUE & 680-709 & & & & \\
\hline -Other Inflammatory Conditions & $690-698$ & 1 & 0.3 & 0.0 & 2.1 \\
\hline $\begin{array}{l}\text { MUSCULOSKELETAL \& CONNECTIVE } \\
\text { TISSUE }\end{array}$ & 710-739 & 7 & 3.6 & 1.7 & 8.0 \\
\hline -Arthropathies & $710-719$ & 4 & 1.9 & 0.6 & 5.3 \\
\hline -Dorsopathies & $720-724$ & 3 & 1.8 & 0.6 & 5.7 \\
\hline $\begin{array}{l}\text { SYMPTOMS, SIGNS, \& ILL-DEFINED } \\
\text { CONDITIONS }\end{array}$ & $780-799$ & 1 & 0.3 & 0.0 & 2.1 \\
\hline -Symptoms & 780-789 & 1 & 0.3 & 0.0 & 2.1 \\
\hline INJURY \& POISONING & 800-999 & 19 & 8.2 & 5.0 & 13.6 \\
\hline -Fracture - Upper Limb & $810-819$ & 2 & 0.8 & 0.2 & 3.4 \\
\hline -Fracture - Lower Limb & 820-829 & 2 & 0.6 & 0.1 & 2.3 \\
\hline -Sprains \& Strains - Back & 846-847 & 2 & 1.0 & 0.2 & 4.7 \\
\hline -Sprains \& Strains - Other & $840-845,848$ & 3 & 1.1 & 0.3 & 3.6 \\
\hline -Open Wound - Upper Limb & 880-887 & 3 & 1.9 & 0.5 & 7.1 \\
\hline -Superficial Injury & 910-919 & 2 & 0.6 & 0.1 & 2.3 \\
\hline -Complications of Surgical/Medical Care & 996-999 & 5 & 2.2 & 0.9 & 5.3 \\
\hline Total & & 28 & 12.4 & 8.3 & 18.7 \\
\hline
\end{tabular}

* Only those diagnostic categories with at least one occurrence appear in this table.

**Standardized to age distribution of 2000 U.S. population. 
Oak Ridge National Laboratory 2010

OSHA Data

Appendix W. Age-Adjusted OSHA Illness and Injury Rates by Diagnostic Category*

Part 3. Men and Women

\begin{tabular}{|c|c|c|c|c|c|}
\hline & & $\begin{array}{l}\text { Number of } \\
\text { Diagnoses }\end{array}$ & $\begin{array}{c}\text { Age-Adjusted } \\
\text { Rate per } 1,000 * *\end{array}$ & \begin{tabular}{|c|} 
Lower $95 \%$ \\
Confidence \\
Limit per 1,000
\end{tabular} & $\begin{array}{c}\text { Upper } 95 \% \\
\text { Confidence } \\
\text { Limit per 1,000 }\end{array}$ \\
\hline Diagnostic Category & ICD-9-CM Code & & & & \\
\hline NERVOUS SYSTEM (NS) \& SENSE ORGANS & 320-389 & 2 & 0.2 & 0.0 & 0.7 \\
\hline -Disorders of Eye & $360-379$ & 1 & 0.1 & 0.0 & 0.7 \\
\hline -Diseases of Ear \& Mastoid & 380-389 & 1 & 0.1 & 0.0 & 0.7 \\
\hline SKIN AND SUBCUTANEOUS TISSUE & 680-709 & 1 & 0.1 & 0.0 & 0.7 \\
\hline -Other Inflammatory Conditions & $690-698$ & 1 & 0.1 & 0.0 & 0.7 \\
\hline $\begin{array}{l}\text { MUSCULOSKELETAL \& CONNECTIVE } \\
\text { TISSUE }\end{array}$ & 710-739 & 23 & 3.5 & 2.3 & 5.5 \\
\hline -Arthropathies & $710-719$ & 11 & 1.8 & 0.9 & 3.4 \\
\hline -Dorsopathies & $720-724$ & 8 & 1.2 & 0.6 & 2.6 \\
\hline -Rheumatism, Excluding Back & 725-729 & 3 & 0.4 & 0.1 & 1.4 \\
\hline -Other Dis \& Acquired Deformities & $730-739$ & 1 & 0.1 & 0.0 & 0.7 \\
\hline $\begin{array}{l}\text { SYMPTOMS, SIGNS, \& ILL-DEFINED } \\
\text { CONDITIONS }\end{array}$ & 780-799 & 2 & 0.3 & 0.1 & 1.1 \\
\hline -Symptoms & 780-789 & 2 & 0.3 & 0.1 & 1.1 \\
\hline INJURY \& POISONING & $800-999$ & 48 & 7.6 & 5.6 & 10.3 \\
\hline -Fracture - Upper Limb & 810-819 & 3 & 0.4 & 0.1 & 1.2 \\
\hline -Fracture - Lower Limb & 820-829 & 2 & 0.2 & 0.0 & 0.7 \\
\hline -Dislocation & 830-839 & 1 & 0.2 & 0.0 & 1.2 \\
\hline -Sprains \& Strains - Back & 846-847 & 6 & 1.1 & 0.4 & 2.5 \\
\hline -Sprains \& Strains - Other & $840-845,848$ & 6 & 0.8 & 0.3 & 2.0 \\
\hline -Open Wound - Head, Neck, Trunk & 870-879 & 3 & 0.5 & 0.2 & 1.8 \\
\hline -Open Wound - Upper Limb & 880-887 & 7 & 1.4 & 0.6 & 3.1 \\
\hline -Superficial Injury & 910-919 & 3 & 0.4 & 0.1 & 1.1 \\
\hline -Contusion & $920-924$ & 4 & 0.7 & 0.2 & 2.0 \\
\hline -Foreign Body Entering Orifice & 930-939 & 2 & 0.2 & 0.0 & 0.7 \\
\hline -Toxic Effects - Non-medicinal & $980-989$ & 2 & 0.4 & 0.1 & 1.8 \\
\hline -Complications of Surgical/Medical Care & 996-999 & 9 & 1.4 & 0.7 & 2.8 \\
\hline Total & & 76 & 11.7 & 9.1 & 14.9 \\
\hline
\end{tabular}

* Only those diagnostic categories with at least one occurrence appear in this table.

**Standardized to age distribution of 2000 U.S. population. 